\title{
Measured Data Used in the Watusi Cross-Section Sets
}

\author{
D. R. Nethaway \\ M. G. Mustafa
}

February 9, 1999

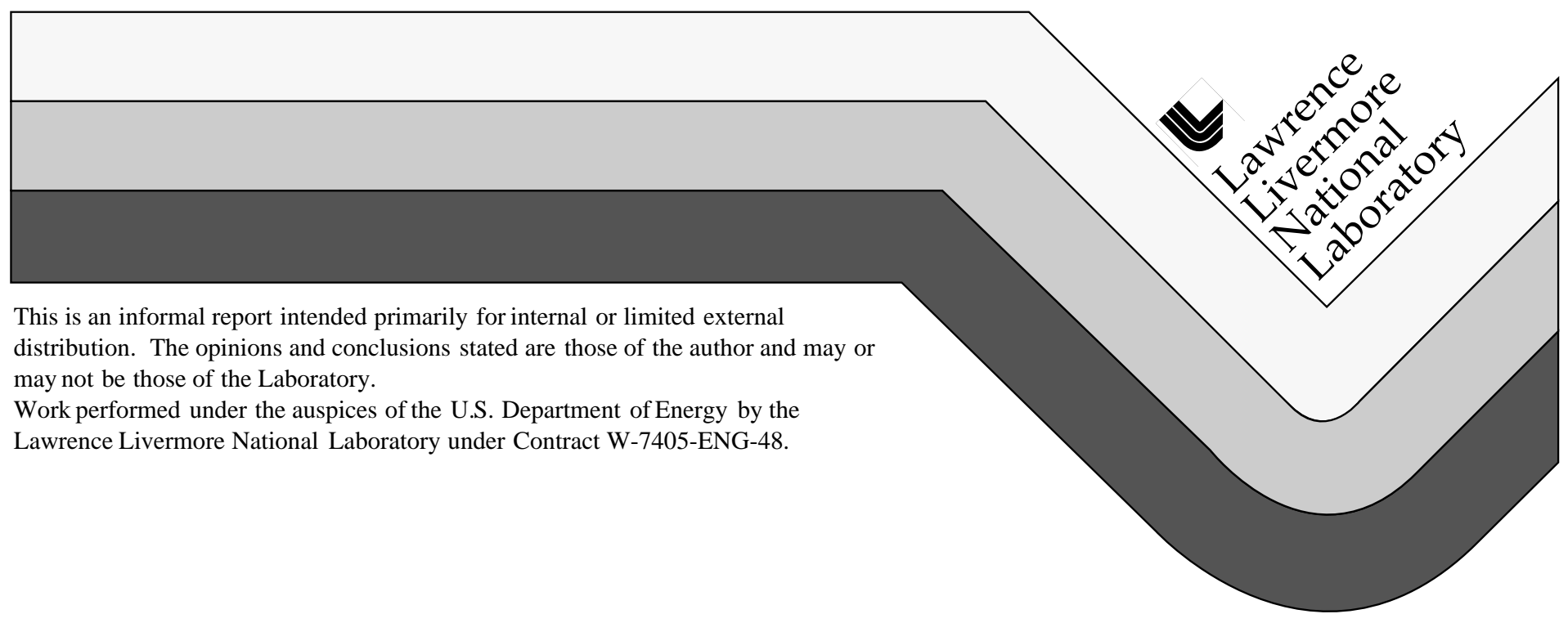




\section{DISCLAIMER}

This document was prepared as an account of work sponsored by an agency of the United States Government. Neither the United States Government nor the University of California nor any of their employees, makes any warranty, express or implied, or assumes any legal liability or responsibility for the accuracy, completeness, or usefulness of any information, apparatus, product, or process disclosed, or represents that its use would not infringe privately owned rights. Reference herein to any specific commercial product, process, or service by trade name, trademark, manufacturer, or otherwise, does not necessarily constitute or imply its endorsement, recommendation, or favoring by the United States Government or the University of California. The views and opinions of authors expressed herein do not necessarily state or reflect those of the United States Government or the University of California, and shall not be used for advertising or product endorsement purposes.

This report has been reproduced directly from the best available copy.

Available to DOE and DOE contractors from the Office of Scientific and Technical Information P.O. Box 62, Oak Ridge, TN 37831

Prices available from (423) 576-8401

Available to the public from the National Technical Information Service

U.S. Department of Commerce 5285 Port Royal Rd. Springfield, VA 22161 


\title{
Measured Data Used in the Watusi Cross-Section Sets
}

\author{
D. R. Nethaway and M. G. Mustafa \\ A Division \\ Lawrence Livermore National Laboratory
}

\section{Introduction}

In this document we list the experimental data that were used to make up the major crosssection sets that we use in the Watusi code to calculate the amount of detector activation in device tests. In order to use experimental data to make up a cross-section set, it is often necessary to extrapolate the cross sections down to either the threshold energy or to 0.01 $\mathrm{keV}$, and to extrapolate up to $20 \mathrm{MeV}$. We then fit the data to a function so that we can get a smoothed set of interpolated values at up to 321 energy points. The combined data are then processed with the Hiroshima code into flux-weighted, group-averaged cross sections for use with the output from the different physics design codes. We typically use the standard 53 or 175 energy group structures.

In a recent companion memo ${ }^{1}$ we described the make up of all of the cross-section sets in detail, giving references to both the experimental data and the theoretical calculations that were used. The following sections have the experimental data, in the form of energy-cross section pairs, for the titanium, chromium, bromine, krypton, yttrium, zirconium, iodine, europium, lutetium, and bismuth sets. The other cross-section sets are not directly based on enough experimental data to warrant their listing here.

Many of the reactions used in these sets are based on calculated cross sections. In making these calculations certain parameters are sometimes adjusted so that the calculated cross sections match experimental data. In some of these cases we have made a further normalization to give a closer agreement to selected experimental data, and such normalizations are noted in this document. In other cases no further normalization was made.

In Table 1 we summarize the reactions for which we present the experimental data given in Tables 2-46. In Figs. 1-35 we show plots of the experimental data together with the actual excitation functions used in the cross-section sets. Some reactions in the current sets are based on preliminary experimental data for which final results are now available. In those cases we show both the preliminary and the final data on the same plots. We expect to find only a few percent change in the calculated activation when we switch to the final experimental data. 
Table 1. List of reactions. Tables 8-11 and 27 have new data that have not been used in the cross-section sets, and therefore are not shown as figures.

\begin{tabular}{|c|c|c|c|c|c|}
\hline \multirow{3}{*}{$\frac{\underline{\text { Reaction }}}{\text { Figure }}$} & Table & $\underline{\text { Figure }}$ & $\underline{\text { Reaction }}$ & \multicolumn{2}{|c|}{ Table } \\
\hline & & & & & \\
\hline & 2,5 & 1 & $89 Y(p, n) 89 g Z r$ & 23 & 16 \\
\hline 47Ti(d,n)48V & 3,6 & 2 & $89 Y(p, n) 89 m Z r$ & 23 & 17 \\
\hline $48 \mathrm{Ti}(\mathrm{d}, 2 \mathrm{n}) 48 \mathrm{~V}$ & 4,7 & 3 & $89 Y(p, 2 n) 88 Z r$ & 24 & 18 \\
\hline 46Ti $(\mathrm{t}, \mathrm{n}) 48 \mathrm{~V}$ & 8 & & $89 Y(d, 2 n) 89 g Z r$ & 25 & 19 \\
\hline $47 \mathrm{Ti}(\mathrm{t}, 2 \mathrm{n}) 48 \mathrm{~V}$ & 8 & & $89 Y(d, 2 n) 89 m Z r$ & 25 & 20 \\
\hline 48Ti(t,a)47Sc & 9 & & $89 Y(d, 3 n) 88 Z r$ & $\begin{array}{r}26,2 \\
8\end{array}$ & 21 \\
\hline 47Ti(d,an)44gSc & 10 & & $89 Y(d, 2 n) 89 Z r$ & 27 & \\
\hline $47 \mathrm{Ti}(\mathrm{d}, \mathrm{an}) 44 \mathrm{mSc}$ & 10 & & $89 Y(n, 2 n) 88 Y$ & 29 & 22 \\
\hline 46Ti(d, a)44gSc & 11 & & $90 Z r(n, 2 n) 89 g Z r$ & 30 & 23 \\
\hline $46 \mathrm{Ti}(\mathrm{d}, \mathrm{a}) 44 \mathrm{mSc}$ & 11 & & $90 Z r(n, 2 n) 89 m Z r$ & 30 & 24 \\
\hline $52 \mathrm{Cr}(p, n) 52 g M n$ & 12 & 4 & $127 I(p, n) 127 X e$ & 31 & 25 \\
\hline $52 \mathrm{Cr}(\mathrm{d}, 2 \mathrm{n}) 52 \mathrm{gMn}$ & 13 & 5 & $127 \mathrm{I}(\mathrm{d}, 2 \mathrm{n}) 127 \mathrm{Xe}$ & 32 & 26 \\
\hline $50 \mathrm{Cr}(\mathrm{d}, \mathrm{a}) 48 \mathrm{~V}$ & 14 & 6 & $127 \mathrm{I}(\mathrm{n}, \mathrm{g}) 128 \mathrm{I}$ & $\begin{array}{l}33,3 \\
4\end{array}$ & 27 \\
\hline $79 B r(p, n) 79 K r$ & 15 & 7 & $151 \mathrm{Eu}(p, n) 151 \mathrm{Gd}$ & $\begin{array}{l}35,3 \\
9\end{array}$ & 28 \\
\hline $79 \mathrm{Br}(\mathrm{d}, 2 \mathrm{n}) 79 \mathrm{Kr}$ & 16 & 8 & $\begin{array}{l}\text { 151Eu(d,2n)151G } \\
d\end{array}$ & $\begin{array}{l}36,4 \\
0\end{array}$ & 29 \\
\hline $81 \mathrm{Br}(p, n) 81 \mathrm{Kr}$ & 17 & 9 & $153 \mathrm{Eu}(p, n) 153 \mathrm{Gd}$ & $\begin{array}{l}37,4 \\
1\end{array}$ & 30 \\
\hline $81 \mathrm{Br}(\mathrm{d}, 2 \mathrm{n}) 81 \mathrm{Kr}$ & 18 & 10 & $\begin{array}{l}\text { 153Eu(d,2n)153G } \\
d\end{array}$ & $\begin{array}{l}38,4 \\
2\end{array}$ & 31 \\
\hline $80 \mathrm{Kr}(\mathrm{n}, \mathrm{g}) 81 \mathrm{Kr}$ & 19 & 11 & 151Eu(n,g)152Eu & 43 & 32 \\
\hline
\end{tabular}




$\begin{array}{lccccr}78 \mathrm{Kr}(\mathrm{n}, 2 \mathrm{n}) 77 \mathrm{Kr} & 20 & 12 & 153 \mathrm{Eu}(\mathrm{n}, \mathrm{g}) 154 \mathrm{Eu} & 44 & 33 \\ 80 \mathrm{Kr}(\mathrm{n}, 2 \mathrm{n}) 79 \mathrm{Kr} & 20 & 13 & 152 \mathrm{Gd}(\mathrm{n}, \mathrm{g}) 153 \mathrm{Gd} & 45 & 34 \\ 78 \mathrm{Kr}(\mathrm{n}, \mathrm{g}) 79 \mathrm{Kr} & 21 & 14 & 154 \mathrm{Gd}(\mathrm{n}, \mathrm{g}) 155 \mathrm{Gd} & 46 & 35 \\ 89 \mathrm{Y}(\mathrm{p}, \mathrm{pn}) 88 \mathrm{Y} & 22 & 15 & & & \end{array}$

$-3-$

\section{Ti0887 Set}

Note that the data given in the following Tables 2-4 from Ref. 2 are preliminary, and the cross-section set should be revised sometime to use the final data given in Tables 5-7, and add the new data in Tables 8-11, all from Ref. 3.

\begin{tabular}{|c|c|c|c|c|c|}
\hline $\mathrm{s}_{\mathrm{p}}(\mathrm{MeV})$ & Sigma (mb) & $\underline{\mathrm{E}}_{\mathrm{p}}(\mathrm{MeV})$ & $\underline{\text { Sigma }(\mathrm{mb})}$ & $\underline{\mathrm{E}}_{\mathrm{p}}(\mathrm{MeV})$ & $\underline{\operatorname{Sigma}(\mathrm{mb})}$ \\
\hline 4.96 & 1.60 & 8.05 & 399. & 17.0 & 205 \\
\hline 5.11 & 4.64 & 8.26 & 424. & 18.0 & 155. \\
\hline 5.27 & 31.0 & 8.89 & 451. & 19.0 & 115. \\
\hline 5.37 & 63.1 & 9.56 & 463. & 20.0 & 89. \\
\hline 5.66 & 134. & 10.82 & 508. & 21.0 & 69. \\
\hline 5.86 & 171. & 11.68 & 508. & 22.0 & 57. \\
\hline 6.37 & 276. & 13.51 & 503. & 23.0 & 47. \\
\hline 6.94 & 309. & 14.56 & 433. & 24.0 & 41. \\
\hline 7.07 & 323. & 15.29 & 350. & 25.0 & 37. \\
\hline 7.48 & 366. & 16.0 & 275. & 26.0 & 34. \\
\hline
\end{tabular}

Table 3. ${ }^{47} \mathrm{Ti}(\mathrm{d}, \mathrm{n})^{48} \mathrm{~V}$ Reaction. Preliminary data from Ref. 2. Note that the data for 8 $\mathrm{MeV}$ and higher are from K. Chen and J. Miller, Phys. Rev. B 134, 1269 (1964), quoted in Ref. 2.

\begin{tabular}{|c|c|c|c|c|c|}
\hline$\underline{E}_{\mathrm{d}}(\mathrm{MeV})$ & Sigma (mb) & $\underline{\mathrm{E}}_{\mathrm{d}}(\mathrm{MeV})$ & $\underline{\operatorname{Sigma}(\mathrm{mb})}$ & $\underline{\mathrm{E}}_{\mathrm{d}}(\mathrm{MeV})$ & $\underline{\text { Sigma }(\mathrm{mb})}$ \\
\hline 1.85 & 1.20 & 3.56 & 136.4 & 7.10 & 261. \\
\hline 2.04 & 3.49 & 3.75 & 167.9 & 8.0 & 208. \\
\hline 2.24 & 7.53 & 3.82 & 190.1 & 9.8 & 122.5 \\
\hline 2.43 & 15.9 & 4.24 & 227. & 12.1 & 93.0 \\
\hline 2.63 & 27.8 & 4.32 & 258. & 14.0 & 70.1 \\
\hline 2.83 & 47.3 & 4.85 & 271. & 15.7 & 63.4 \\
\hline 3.03 & 59.2 & 5.00 & 299. & 17.6 & 51.9 \\
\hline 3.21 & 83.2 & 5.83 & 296. & 19.5 & 42.2 \\
\hline 3.33 & 105.4 & 6.38 & 282. & 20.5 & 42.0 \\
\hline
\end{tabular}




\begin{tabular}{|c|c|c|c|c|c|}
\hline$E_{\mathrm{d}}(\mathrm{MeV})$ & Sigma (mb) & $\underline{E}_{\mathrm{d}}(\mathrm{MeV})$ & $\underline{\operatorname{Sigma}(\mathrm{mb})}$ & $\underline{E}_{\mathrm{d}}(\mathrm{MeV})$ & $\underline{\operatorname{Sigma}(\mathrm{mb})}$ \\
\hline 7.60 & 6.1 & 10.41 & 194.5 & 15.50 & 432. \\
\hline 8.10 & 16.8 & 10.56 & 203.9 & 17.04 & 451. \\
\hline 8.23 & 22.4 & 11.00 & 264 . & 18.48 & 440. \\
\hline 8.69 & 55.7 & 11.75 & 289. & 19.84 & 417. \\
\hline 9.26 & 95.9 & 12.49 & 351. & & \\
\hline 9.74 & 163.5 & 14.29 & 414. & & \\
\hline
\end{tabular}

Table 5. ${ }^{48} \mathrm{Ti}(\mathrm{p}, \mathrm{n})^{48} \mathrm{~V}$ Reaction. Final results from Ref. 3.

\begin{tabular}{|c|c|c|c|c|c|}
\hline$\underline{E}_{p}(\mathrm{MeV})$ & Sigma (mb) & $\underline{\mathrm{E}}_{\mathrm{p}}(\mathrm{MeV})$ & $\underline{\text { Sigma }(\mathrm{mb})}$ & $\underline{\mathrm{E}}_{\mathrm{p}}(\mathrm{MeV})$ & $\underline{\text { Sigma }(\mathrm{mb}}$ \\
\hline-4.96 & $1.60+/-0.17$ & 7.48 & $366 .+/-11$. & 15.90 & $291 .+/-9$. \\
\hline 5.11 & $4.64+/-0.48$ & 8.05 & 399. +/- 12. & 17.72 & 165. +/- 5 . \\
\hline 5.27 & $31.0+/-1.0$ & 8.26 & $424 .+/-13$. & 19.37 & 106. +/- 3. \\
\hline 5.37 & $63.1+/-2.0$ & 8.89 & 451. +/- 14. & 20.95 & $74.8+/-2.4$ \\
\hline 5.66 & 134. +/- 4 & 9.56 & 463. +/- 14. & 22.65 & $58.0+/-1.8$ \\
\hline 5.86 & 171. +/- 5 . & 10.82 & 508. +/- 15. & 24.05 & $45.8+/-1.5$ \\
\hline 6.37 & 276. +/- 8. & 11.68 & 508. +/- 15 . & 25.41 & $42.0+/-1.3$ \\
\hline 6.94 & 309. +/- 9 . & 13.20 & 504. +/- 16. & 26.71 & $39.3+/-1.3$ \\
\hline 7.07 & 323. +/- 10. & 15.00 & $371 .+/-12$. & & \\
\hline
\end{tabular}
$\mathrm{MeV}$.

Table 6. ${ }^{47} \operatorname{Ti}(\mathrm{d}, \mathrm{n})^{48} \mathrm{~V}$ Reaction. Final results from Ref. 3. The data extend up to 34.6

\begin{tabular}{|c|c|c|c|c|c|}
\hline$\underline{E}_{\mathrm{d}}(\mathrm{MeV})$ & Sigma (mb) & $\underline{E}_{\mathrm{d}}(\mathrm{MeV})$ & Sigma (mb) & $\underline{E}_{\mathrm{d}}(\mathrm{MeV})$ & Sigma (mb) \\
\hline 1.85 & $1.20+/-0.04$ & 3.82 & 190. +/- 6 & 9.35 & $177 .+/-5$. \\
\hline 2.04 & $3.49+/-0.11$ & 3.91 & 214. +/- 7 . & 10.70 & 128. +/- 4. \\
\hline 2.24 & $7.53+/-0.2$ & 4.24 & 227. +/- 7 . & 11.92 & $115 .+/-4$. \\
\hline 2.43 & $15.9+/-0.5$ & 4.32 & 258. +/- 8. & 13.04 & $96.3+/-3.0$ \\
\hline 2.63 & $27.8+/-0.9$ & 4.85 & 271. +/- 9. & 14.13 & $78.0+/-2.5$ \\
\hline 2.83 & $47.3+/-1.5$ & 5.00 & 299. +/- 10. & 15.16 & $68.9+/-2.2$ \\
\hline 3.03 & $59.2+/-1.9$ & 5.83 & 296. +/- 9 . & 16.14 & $69.8+/-2.2$ \\
\hline 3.21 & $83.2+/-2.6$ & 6.13 & 286. +/- 9. & 17.06 & $61.8+/-1.9$ \\
\hline 3.33 & 105. +/- 3 & 6.38 & 282. +/- 9. & 17.94 & $57.0+/-1.8$ \\
\hline 3.56 & 136. +/- 4. & 7.10 & 261. +/- 8. & 21.94 & $45.7+/-1.5$ \\
\hline
\end{tabular}



$\mathrm{MeV}$.

Table 7. ${ }^{48} \mathrm{Ti}(\mathrm{d}, 2 \mathrm{n}){ }^{48} \mathrm{~V}$ Reaction. Final results from Ref. 3. The data extend up to 34.6

\begin{tabular}{|c|c|c|c|c|c|}
\hline $\begin{array}{l}\underline{\mathrm{E}}_{\mathrm{d}}(\mathrm{MeV}) \\
(\mathrm{mb})\end{array}$ & Sigma (mb) & $\underline{\mathrm{E}}_{\mathrm{d}}(\mathrm{MeV})$ & $\underline{\text { Sigma }(\mathrm{mb})}$ & $\underline{E}_{\mathrm{d}}(\mathrm{MeV})$ & Sigma \\
\hline 7.46 & $0.666+/-0.021$ & 9.74 & 162. +/- 5 . & 15.50 & 431. +/- 14 \\
\hline 7.60 & $3.70+/-0.12$ & 10.01 & 165. +/- 5 . & 15.99 & $421 .+/-13$ \\
\hline 7.82 & $5.43+/-0.17$ & 10.56 & 203. +/- 6 . & 17.04 & 450. +/- 14 \\
\hline 8.10 & $15.50+/-0.49$ & 10.86 & $230 .+/-7$. & 17.95 & 434. +/- 14 \\
\hline 8.23 & $19.85+/-0.63$ & 11.00 & 264. +/- 8. & 18.48 & 440. +/- 14 \\
\hline 8.24 & $19.35+/-0.61$ & 11.75 & 287. +/- 9 . & 19.84 & 417. +/- 13 \\
\hline 8.69 & $53.6+/-1.7$ & 11.95 & 303. +/- 10 . & 19.92 & 400. $+/-13$ \\
\hline 8.84 & $58.1+/-1.8$ & 12.49 & 348. +/- 11. & 21.73 & $368 .+/-12$ \\
\hline 9.26 & $93.9+/-3$ & 13.93 & $391 .+/-12$. & 23.35 & $318 .+/-10$ \\
\hline 9.33 & 100. +/- 3 & 14.29 & 412. +/- 13. & 24.89 & $261 .+/-8$ \\
\hline
\end{tabular}

Table 8. ${ }^{\mathrm{Nat}} \mathrm{Ti}(\mathrm{t}, \mathrm{xn})^{48} \mathrm{~V}$ Reaction. Final results from Ref. 3. The data in column 2 were split into individual cross sections for the ${ }^{46} \mathrm{Ti}(\mathrm{t}, \mathrm{n}){ }^{48} \mathrm{~V}(\mathrm{col} .3)$ and ${ }^{47} \mathrm{Ti}(\mathrm{t}, 2 \mathrm{n})^{48} \mathrm{~V}$ (col. 4) reactions using the results of Stapre calculations to scale the measured values.

\begin{tabular}{rccc}
$\mathrm{E}_{\mathrm{t}}(\mathrm{MeV})$ & \multicolumn{1}{c}{ Sigma $(\mathrm{mb})$} & ${ }^{46} \mathrm{Ti}(\mathrm{t}, \mathrm{n})^{48} \mathrm{~V}$ & \\
1.67 & $0.010+/-0.001$ & 0.125 & ${ }^{47} \mathrm{Ti}(\mathrm{t}, 2 \mathrm{n})^{48} \mathrm{~V}$ \\
2.03 & $0.155+/-0.023$ & 2.42 & 0.0 \\
2.21 & $0.199+/-0.010$ & 11.7 & 0.076 \\
2.73 & $1.05+/-0.04$ & 40.8 & 1.57 \\
3.25 & $4.01+/-0.13$ & 70.2 & 10.3 \\
3.77 & $8.46+/-0.26$ & 95.3 & 38.9 \\
4.59 & $16.5+/-0.5$ & 83.0 & 122. \\
6.62 & $31.4+/-1.0$ & 64.2 & 340. \\
8.13 & $37.3+/-1.1$ & 51.1 & 441. \\
9.50 & $40.5+/-1.2$ & 40.3 & 499. \\
10.91 & $42.8+/-1.3$ & 30.6 & 541. \\
12.45 & $41.9+/-1.3$ & 24.8 & 540. \\
13.88 & $40.4+/-1.3$ & 20.1 & 526. \\
15.32 & $37.7+/-1.2$ & 16.7 & 494. \\
16.54 & $33.9+/-1.3$ & 13.7 & 446. \\
17.71 & $29.4+/-1.8$ & 9.93 & 387.
\end{tabular}


Table 9. ${ }^{48} \mathrm{Ti}(\mathrm{t}, \mathrm{a}){ }^{47} \mathrm{Sc}$ Reaction. Final results from Ref. 3.

\begin{tabular}{|c|c|c|c|c|c|}
\hline$\frac{\mathrm{E}_{\mathrm{t}}(\mathrm{MeV})}{(\mathrm{mb})}$ & Sigma (mb) & $\underline{\mathrm{E}}_{\mathrm{t}}(\mathrm{MeV})$ & Sigma (mb) & $\underline{\mathrm{E}}_{\mathrm{t}}(\mathrm{MeV})$ & Sigma \\
\hline 1.67 & $\begin{array}{l}0.014+/- \\
0.001\end{array}$ & 3.77 & $9.59+/-0.3$ & 9.50 & $55.1+/-1.7$ \\
\hline 2.04 & $\begin{array}{l}0.152+/- \\
0.008\end{array}$ & 4.28 & $15.8+/-.5$ & 10.30 & $53.1+/-1.7$ \\
\hline 2.21 & $0.27+/-0.01$ & 4.59 & $18.2+/-0.6$ & 12.47 & $44.3+/-1.4$ \\
\hline 2.60 & $1.14+/-0.04$ & 5.73 & $28.7+/-0.9$ & 13.61 & $40.3+/-1.3$ \\
\hline 2.73 & $1.56+/-0.05$ & 6.63 & $37.1+/-1.2$ & 15.19 & $34.2+/-1.0$ \\
\hline 3.08 & $3.71+/-0.12$ & 7.23 & $42.5+/-1.3$ & 16.55 & $33.1+/-1.0$ \\
\hline 3.25 & $4.79+/-0.15$ & 8.15 & $48.4+/-1.5$ & 17.83 & $32.6+/-1.0$ \\
\hline 3.62 & $8.21+/-0.26$ & 8.87 & $50.6+/-1.6$ & & \\
\hline
\end{tabular}

$-6-$

Table 10. ${ }^{47} \mathrm{Ti}(\mathrm{d}, \mathrm{an}){ }^{44 \mathrm{~g},}{ }^{44 \mathrm{~m}} \mathrm{Sc}$ Reaction. Final results from Ref. 3.

\begin{tabular}{|c|c|c|}
\hline$\underline{E}_{d}(\mathrm{MeV})$ & ${ }^{44 \mathrm{~g}} \mathrm{Sc}$ Sigma $(\mathrm{mb})$ & ${ }^{44 \mathrm{~m}} \mathrm{Sc}$ Sigma $(\mathrm{mb})$ \\
\hline 9.35 & $0.036+/-0.010$ & $0.004+/-0.007$ \\
\hline 10.70 & $0.50+/-0.03$ & $0.277+/-0.016$ \\
\hline 11.92 & $4.6+/-0.14$ & $2.35+/-0.07$ \\
\hline 11.92 & $4.17+/-0.14$ & $2.60+/-0.1$ \\
\hline 13.04 & $12.8+/-0.4$ & $7.35+/-0.2$ \\
\hline 14.13 & $22.0+/-0.7$ & $12.1+/-0.4$ \\
\hline 15.16 & $32.7+/-0.9$ & $17.3+/-0.5$ \\
\hline 16.14 & $44.8+/-1.5$ & $23.6+/-0.7$ \\
\hline 17.06 & $52.8+/-1.5$ & $26.4+/-0.8$ \\
\hline 17.94 & $58.4+/-1.8$ & $30.2+/-0.9$ \\
\hline
\end{tabular}

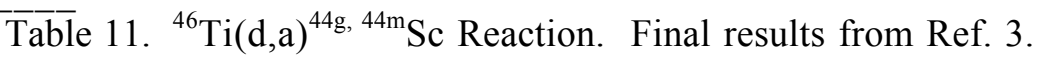




\begin{tabular}{|c|c|c|}
\hline$\underline{E}_{\mathrm{d}}(\mathrm{MeV})$ & ${ }^{44 \mathrm{~g}} \mathrm{Sc}$ Sigma $(\mathrm{mb})$ & ${ }^{44 \mathrm{~m}} \mathrm{Sc} \operatorname{Sigma}(\mathrm{mb})$ \\
\hline 4.85 & & $1.01+/-0.1$ \\
\hline 5.06 & $19.8+/-0.7$ & $1.99+/-0.14$ \\
\hline 5.36 & $23.9+/-0.8$ & $2.38+/-0.1$ \\
\hline 5.92 & $31.6+/-1.1$ & $2.95+/-0.14$ \\
\hline 6.38 & & $3.35+/-0.2$ \\
\hline 7.67 & $48.5+/-1.6$ & $6.91+/-0.3$ \\
\hline 8.10 & & $7.38+/-0.4$ \\
\hline 9.20 & $62.0+/-2.1$ & $11.9+/-0.7$ \\
\hline 9.74 & & $13.5+/-0.5$ \\
\hline 10.41 & & $12.9+/-0.5$ \\
\hline 10.54 & $63.4+/-2.0$ & $15.1+/-0.6$ \\
\hline 11.00 & & $15.1+/-0.6$ \\
\hline 11.51 & $57.7+/-2.0$ & $16.9+/-0.7$ \\
\hline 11.75 & & $15.1+/-0.7$ \\
\hline 12.49 & & $16.8+/-0.7$ \\
\hline 13.37 & $46.4+/-2.0$ & $15.9+/-0.8$ \\
\hline 14.29 & & $15.9+/-1.1$ \\
\hline 15.10 & $33.0+/-2.1$ & $14.1+/-1.1$ \\
\hline 16.63 & $23.5+/-2.6$ & $10.2+/-1.2$ \\
\hline 18.26 & $19.0+/-4.0$ & $6.9+/-1.4$ \\
\hline 19.83 & $17.0+/-6.0$ & $6.9+/-2.9$ \\
\hline
\end{tabular}

$-7-$

\section{Cr0386 Set}

The ${ }^{50} \mathrm{Cr}(\mathrm{n}, \mathrm{t})^{48} \mathrm{~V}$ reaction is based on the average $(0.0875 \mathrm{mb})$ of results given in Ref. 4 $(0.066 \mathrm{mb})$ and in Ref. $5(0.109 \mathrm{mb})$, both at $14.6 \mathrm{MeV}$. The ${ }^{50} \mathrm{Cr}(\mathrm{n}, \mathrm{np})^{49} \mathrm{~V}$ reaction is based on a measurement given in Ref. $6(405+/-65 \mathrm{mb}$ at $14.7 \mathrm{MeV})$.

Table 12. ${ }^{52} \mathrm{Cr}(\mathrm{p}, \mathrm{n}){ }^{52 \mathrm{~g}} \mathrm{Mn}$ Reaction. Final results from Ref. 7.

\begin{tabular}{|c|c|c|c|c|c|}
\hline$\underline{E}_{\mathrm{p}}(\mathrm{MeV})$ & Sigma (mb) & $\underline{\mathrm{E}}_{\mathrm{p}}(\mathrm{MeV})$ & Sigma (mb) & $\underline{E}_{p}(\mathrm{MeV})$ & Sigma (mb) \\
\hline 6.30 & 4.44 & 9.84 & 80.9 & 18.90 & 61.1 \\
\hline 6.75 & 14.14 & 11.82 & 111.7 & 20.91 & 44.4 \\
\hline 7.32 & 26.0 & 14.24 & 138.2 & 22.91 & 35.7 \\
\hline 7.79 & 41.1 & 15.39 & 120.7 & 24.91 & 29.1 \\
\hline 8.78 & 56.9 & 16.89 & 96.1 & 26.91 & 24.6 \\
\hline
\end{tabular}




\begin{tabular}{|c|c|c|c|c|c|}
\hline$\underline{E}_{\mathrm{d}}(\mathrm{MeV})$ & Sigma (mb) & $\underline{E}_{\mathrm{d}}(\mathrm{MeV})$ & Sigma (mb) & $\underline{E}_{\mathrm{d}}(\mathrm{MeV})$ & $\underline{\text { Sigma }(\mathrm{mb})}$ \\
\hline 8.17 & 0.176 & 9.97 & 34.15 & 15.89 & 181.6 \\
\hline 8.27 & 0.508 & 10.62 & 58.3 & 16.91 & 187.0 \\
\hline 8.37 & 0.961 & 10.97 & 74.6 & 17.66 & 192.9 \\
\hline 8.46 & 1.84 & 11.47 & 87.4 & 17.80 & 197.2 \\
\hline 8.56 & 2.48 & 11.75 & 100.6 & 18.25 & 198.5 \\
\hline 8.71 & 4.71 & 11.76 & 97.5 & 18.83 & 198.3 \\
\hline 8.97 & 7.99 & 13.47 & 141.3 & 19.32 & 195.5 \\
\hline 9.46 & 17.29 & 13.78 & 150.1 & 19.58 & 197.0 \\
\hline 9.60 & 25.03 & 15.05 & 169.8 & 19.82 & 197.6 \\
\hline 9.89 & 31.30 & 15.82 & 177.2 & & \\
\hline
\end{tabular}

Table 14. ${ }^{50} \mathrm{Cr}(\mathrm{d}, \mathrm{a}){ }^{48} \mathrm{~V}$ Reaction. Final results from Ref. 8.

\begin{tabular}{|c|c|c|c|c|c|}
\hline$\frac{\underline{\mathrm{E}}_{\mathrm{d}}(\mathrm{MeV})}{(\mathrm{mb})}$ & Sigma (mb) & $\underline{E}_{d}(\mathrm{MeV})$ & Sigma (mb) & $\underline{\mathrm{E}}_{\mathrm{d}}(\mathrm{MeV})$ & Sigma \\
\hline 4.59 & $9.2+/-0.16$ & 8.97 & $51.3+/-0.6$ & 13.47 & $53.5+/-3.4$ \\
\hline 4.64 & $8.78+/-0.17$ & 9.46 & $54.1+/-0.7$ & 14.90 & $40.8+/-3.5$ \\
\hline 5.78 & $19.3+/-0.6$ & 9.60 & $61.8+/-3.0$ & 15.05 & $40.6+/-4.5$ \\
\hline 6.97 & $31.9+/-1.0$ & 9.62 & $59.8+/-4.0$ & 15.89 & $34.4+/-1.0$ \\
\hline 7.64 & $38.2+/-0.45$ & 9.89 & $57.8+/-1.4$ & 16.91 & $30.9+/-6.2$ \\
\hline 8.17 & $41.2+/-0.7$ & 9.97 & $60.4+/-1.4$ & 17.66 & $25.1+/-0.7$ \\
\hline 8.27 & $43.4+/-0.8$ & 10.62 & $64.4+/-3.3$ & 18.25 & $25.8+/-5.0$ \\
\hline 8.37 & $44.6+/-0.8$ & 10.97 & $62.4+/-2.2$ & 18.83 & $26.0+/-3.5$ \\
\hline 8.46 & $48.1+/-0.3$ & 11.47 & $61.5+/-3.3$ & 19.32 & $22.8+/-0.7$ \\
\hline 8.56 & $47.5+/-0.7$ & 11.76 & $63.0+/-3.2$ & 19.58 & $20.2+/-3.2$ \\
\hline 8.71 & $50.7+/-0.3$ & 12.89 & $57.3+/-3.5$ & 19.82 & $17.1+/-3.9$ \\
\hline
\end{tabular}

\section{Br0391 Set}

Table 15. ${ }^{79} \mathrm{Br}(\mathrm{p}, \mathrm{n}){ }^{79} \mathrm{Kr}$ Reaction. Final results from Ref. 9. The results in columns 2 and 5 were scaled down by the factor, 0.928 , to correct to the photon abundances in use for Test Program measurements, and are given in columns 3 and 6.

\begin{tabular}{|c|c|c|c|c|c|}
\hline$\underline{\mathrm{E}_{\mathrm{p}}(\mathrm{MeV})}$ & Sigma (mb) & $\underline{\text { Scaled }(\mathrm{mb})}$ & $\underline{\mathrm{E}}_{\mathrm{p}}(\mathrm{MeV})$ & Sigma (mb) & $\underline{\text { Scaled }}$ \\
\hline 2.48 & $0.26+/-0.02$ & 0.24 & 9.54 & $634 .+/-19$. & 588. \\
\hline
\end{tabular}




$\begin{array}{rrrrrr}2.80 & 2.22+/-0.04 & 2.06 & 10.06 & 693 .+/-21 . & 643 . \\ 3.17 & 8.18+/-0.05 & 7.59 & 10.27 & 670 .+/-20 . & 621 . \\ 3.47 & 19.6+/-0.9 & 18.2 & 10.41 & 700 .+-21 . & 649 . \\ 3.94 & 47.2+/-1.4 & 43.8 & 10.98 & 735 .+/-22 . & 682 . \\ 4.53 & 91.5+/-2.8 & 84.9 & 11.09 & 704 .+/-21 . & 653 . \\ 4.88 & 120.3+/-3.6 & 111.6 & 11.37 & 704 .+/-21 . & 653 . \\ 5.55 & 188.0+/-5.6 & 174.4 & 11.74 & 726 .+/-22 . & 673 . \\ 6.29 & 308 .+/-9 . & 286 . & 12.29 & 684 .+/-21 . & 634 . \\ 6.87 & 360 .+/-11 . & 334 . & 12.36 & 670 .+/-20 . & 621 . \\ 6.99 & 402 .+/-12 . & 373 . & 12.96 & 585 .+/-18 . & 543 . \\ 7.05 & 402 .+/-12 . & 373 . & 13.53 & 502 .+/-15 . & 466 . \\ 8.10 & 520 .+/-16 . & 482 . & 14.35 & 379 .+/-11 . & 352 . \\ 8.36 & 545 .+/-16 . & 506 . & 14.66 & 345 .+/-10 . & 320 . \\ 9.06 & 607 .+/-18 . & 563 . & 15.65 & 238 .+/-7 . & 221 . \\ 9.11 & 628 .+/-19 & 583 . & 16.87 & 149.6+/-4.5 & 138.8\end{array}$

Table 16. ${ }^{79} \mathrm{Br}(\mathrm{d}, 2 \mathrm{n}){ }^{79} \mathrm{Kr}$ Reaction. Final results from Ref. 9. The results in columns 2 and 5 were scaled down by the factor, 0.928 , to correct to the photon abundances in use for Test Program measurements, and are given in columns 3 and 6.

\begin{tabular}{|c|c|c|c|c|c|}
\hline$\frac{\underline{\mathrm{E}}_{\mathrm{d}}(\mathrm{MeV})}{(\mathrm{mb})}$ & $\underline{\text { Sigma (mb) }}$ & $\underline{\text { Scaled }(\mathrm{mb})}$ & $\underline{\mathrm{E}}_{\mathrm{d}}(\mathrm{MeV})$ & $\underline{\text { Sigma }(\mathrm{mb})}$ & $\underline{\text { Scaled }}$ \\
\hline 5.04 & $0.64+/-0.11$ & 0.59 & 9.44 & 574. +/- 17. & 532. \\
\hline 5.09 & $1.20+/-0.20$ & 1.11 & 9.77 & 606. +/- 18 & 562. \\
\hline 5.19 & $3.39+/-0.35$ & 3.14 & 9.85 & 627. +/- 19. & 582. \\
\hline 5.50 & $20.2+/-0.65$ & 18.7 & 10.27 & 619. +/- 19. & 574. \\
\hline 5.51 & $21.3+/-0.6$ & 19.8 & 10.87 & 720. +/- 22. & 668. \\
\hline 5.93 & $57.5+/-1.7$ & 53.3 & 11.86 & 722. +/- 22. & 670. \\
\hline 6.05 & $69.3+/-2.1$ & 64.3 & 12.24 & 806. +/- 24. & 748. \\
\hline 6.23 & $91.7+/-2.8$ & 85.1 & 13.20 & 805. +/- 24 & 747. \\
\hline 6.53 & $159.7+/-4.8$ & 148.1 & 13.27 & 805. +/- 24. & 747. \\
\hline 6.85 & $200.3+/-6.0$ & 185.8 & 13.55 & $841 .+/-25$. & 780. \\
\hline 7.32 & 261. +/- 8 & 242. & 14.46 & 817. +/- 25. & 758. \\
\hline 7.55 & 317. +/- 10. & 294. & 14.57 & 883. +/- 27. & 819. \\
\hline 7.76 & $341 .+/-10$. & 316. & 15.64 & 842. +/- 25. & 781. \\
\hline 8.67 & 484. +/- 15 & 449. & 16.75 & 850. +/- 26. & 788. \\
\hline 9.36 & 576. +/- 17 & 534. & & & \\
\hline
\end{tabular}

Table 17. ${ }^{81} \mathrm{Br}(\mathrm{p}, \mathrm{n}){ }^{81} \mathrm{Kr}$ Reaction. Preliminary data from Ref. 10 (actually used in the Br0391 cross-section set) in columns 2 and 5, and final data from Ref. 9 (not used yet) in Columns 3 and 6.

Ref. $10 \quad$ Ref. 9

Ref. 10

Ref. 9 


\begin{tabular}{|c|c|c|c|c|c|}
\hline$\frac{E_{p}(\mathrm{MeV})}{(\mathrm{mb})}$ & $\underline{\operatorname{Sigma}(\mathrm{mb})}$ & $\underline{\text { Sigma (mb) }}$ & $\underline{\mathrm{E}}_{\mathrm{p}}(\mathrm{MeV})$ & $\underline{\operatorname{Sigma}(\mathrm{mb})}$ & $\underline{\text { Sigma }}$ \\
\hline 2.23 & 0.44 & $0.47+/-0.02$ & 9.06 & 616. & $660 .+/-26$. \\
\hline 2.80 & 2.70 & $2.90+/-0.09$ & 9.11 & & $705 .+/-28$. \\
\hline 3.17 & 8.07 & $8.66+/-0.35$ & 9.54 & 656. & 704. +/- 28. \\
\hline 3.94 & 41.9 & $45.0+/-1.8$ & 10.06 & & 724. +/- 29. \\
\hline 4.53 & 109.2 & $117.1+/-4.7$ & 10.98 & 627. & 617. +/- 25. \\
\hline 4.88 & 136.7 & $146.4+/-5.9$ & 11.09 & & 578. +/- 23. \\
\hline 5.55 & 196. & 210. +/- 8 & 12.29 & 404. & 434. +/- 17. \\
\hline 6.29 & 335. & 359. +/- 14. & 12.36 & & 401. +/- 16. \\
\hline 6.87 & 418. & 448. +/- 18. & 13.52 & 259. & 278. +/- 11 \\
\hline 6.99 & & 456. +/- 18. & 14.35 & & $193.3+/-7.7$ \\
\hline 7.05 & 400. & 429. +/- 17. & 15.65 & & $125.4+/-5.0$ \\
\hline 8.10 & & 579. +/- 23. & 16.87 & & $79.7+/-3.1$ \\
\hline 8.36 & 591. & $634 .+/-25$. & & & \\
\hline
\end{tabular}

Table 18. ${ }^{81} \mathrm{Br}(\mathrm{d}, 2 \mathrm{n})^{81} \mathrm{Kr}$ Reaction. Preliminary data from Ref. 10 (actually used in the Br0391 cross-section set) in columns 2 and 5, and final data from Ref. 9 (not used yet) in Columns 3 and 6. Ref. $10 \quad$ Ref. 9

\begin{tabular}{|c|c|c|c|c|c|}
\hline$\frac{\mathrm{E}_{\mathrm{d}}(\mathrm{MeV})}{(\mathrm{mb})}$ & $\underline{\operatorname{Sigma}(\mathrm{mb})}$ & $\underline{\text { Sigma (mb) }}$ & $\underline{\mathrm{E}}_{\mathrm{d}}(\mathrm{MeV})$ & $\underline{\operatorname{Sigma}(\mathrm{mb})}$ & $\underline{\text { Sigma }}$ \\
\hline 3.83 & & $0.018+/-0.009$ & 8.67 & 649. & 691. +/- 28. \\
\hline 4.52 & & $14.6+/-0.9$ & 9.36 & & $745 .+/-30$. \\
\hline 4.76 & & $32.8+/-1.5$ & 9.44 & 722. & $775 .+/-31$. \\
\hline 4.95 & 43.2 & $42.5+/-1.7$ & 9.85 & 779. & $836 .+/-33$. \\
\hline 5.05 & 59.6 & $60.4+/-2.4$ & 10.27 & 784. & $841 .+/-34$. \\
\hline 5.19 & 76.5 & $79.5+/-3.2$ & 10.87 & & $880 .+/-35$. \\
\hline 5.50 & 117.2 & $124.7+/-5.0$ & 11.86 & 815. & $875 .+/-35$. \\
\hline 5.51 & 119.1 & $127.3+/-5.1$ & 12.24 & & $950 .+/-38$. \\
\hline 5.93 & 178.6 & $191.6+/-7.7$ & 13.27 & 913. & $980 .+/-39$. \\
\hline 6.23 & 231. & 247. +/- 10 & 13.55 & & $965 .+/-39$. \\
\hline 6.53 & 301. & 322. +/- 13. & 14.46 & & 911. +/- 36 \\
\hline 6.85 & 356. & 388. +/- 16. & 14.57 & 964. & 1034. +/- 41 \\
\hline 7.32 & 414. & $444 .+/-18$ & 15.64 & & 895. +/- 36 \\
\hline 7.55 & 477. & 512. +/- 20. & 16.75 & & 876. +/- 35 . \\
\hline
\end{tabular}

\section{$\underline{\text { Kr0991 Set }}$}




\begin{tabular}{|c|c|c|c|c|c|}
\hline $\begin{array}{l}\underline{\mathrm{E}}_{\mathrm{n}}(\mathrm{keV}) \\
(\mathrm{mb})\end{array}$ & $\underline{\text { Sigma (mb) }}$ & $\underline{E}_{\mathrm{n}}(\mathrm{keV})$ & Sigma (mb) & $\underline{E}_{\mathrm{n}}(\mathrm{keV})$ & Sigma \\
\hline 5.0 & $\begin{array}{l}457.6+/- \\
137.5\end{array}$ & 37.5 & $249.7+/-7.1$ & 130.0 & $139.4+/-2.8$ \\
\hline 6.0 & $\begin{array}{l}418.9+/- \\
102.6\end{array}$ & 40.0 & $284.7+/-7.0$ & 140.0 & $144.2+/-3.5$ \\
\hline 7.0 & $506.9+/-81.6$ & 42.5 & $257.0+/-6.3$ & 150.0 & $95.7+/-5.2$ \\
\hline 8.0 & $438.7+/-66.9$ & 45.0 & $206.3+/-6.1$ & 160.0 & $103.2+/-9.2$ \\
\hline 9.0 & $361.6+/-56.1$ & 47.5 & $209.3+/-6.3$ & 170.0 & $115.2+/-9.6$ \\
\hline 10.0 & $369.9+/-37.3$ & 50.0 & $204.2+/-5.3$ & 180.0 & $111.6+/-9.5$ \\
\hline 12.0 & $357.0+/-26.8$ & 55.0 & $224.4+/-4.7$ & 196.1 & $\begin{array}{l}117.6+/- \\
11.6\end{array}$ \\
\hline 14.0 & $417.7+/-21.1$ & 60.0 & $191.4+/-4.4$ & 199.8 & $\begin{array}{l}115.4+/- \\
11.0\end{array}$ \\
\hline 16.0 & $370.8+/-16.0$ & 65.0 & $169.2+/-3.9$ & 203.6 & $\begin{array}{l}119.2+/- \\
10.8\end{array}$ \\
\hline 18.0 & $353.2+/-14.3$ & 70.0 & $172.3+/-3.8$ & 207.5 & $\begin{array}{l}104.2+/- \\
10.9\end{array}$ \\
\hline 20.0 & $350.1+/-16.2$ & 75.0 & $166.9+/-3.9$ & 211.6 & $\begin{array}{l}89.0+/- \\
10.9\end{array}$ \\
\hline 22.0 & $357.8+/-14.1$ & 80.0 & $146.8+/-3.7$ & 215.7 & $\begin{array}{l}92.0+/- \\
10.6\end{array}$ \\
\hline 24.0 & $336.9+/-11.8$ & 85.0 & $160.1+/-3.6$ & 220.0 & $\begin{array}{l}104.1+/- \\
10.9\end{array}$ \\
\hline 26.0 & $347.7+/-10.5$ & 90.0 & $152.2+/-3.6$ & 224.4 & $\begin{array}{l}99.5+/- \\
10.7^{-}\end{array}$ \\
\hline 28.0 & $302.9+/-10.5$ & 95.0 & $142.5+/-4.0$ & 229.0 & $\begin{array}{l}105.5+/- \\
11.0\end{array}$ \\
\hline 30.0 & $308.1+/-8.6$ & 100.0 & $149.5+/-3.1$ & 233.7 & $11.3^{97.9+/-}$ \\
\hline 32.5 & $249.6+/-7.8$ & 110.0 & $143.4+/-2.8$ & 238.5 & $\begin{array}{l}95.7+/- \\
11.5\end{array}$ \\
\hline 35.0 & $269.5+/-8.2$ & 120.0 & $141.9+/-2.6$ & 243.0 & $\begin{array}{l}96.2+/- \\
11.8\end{array}$ \\
\hline
\end{tabular}

Table 20. ${ }^{78} \mathrm{Kr}(\mathrm{n}, 2 \mathrm{n}){ }^{77} \mathrm{Kr}$ and ${ }^{80} \mathrm{Kr}(\mathrm{n}, 2 \mathrm{n}){ }^{79} \mathrm{Kr}$ Reactions. Final results from Ref. 12.

\begin{tabular}{|c|c|c|}
\hline$\underline{E}_{n}(\mathrm{MeV})$ & ${ }^{78} \mathrm{Kr}$ Sigma $(\mathrm{mb})$ & ${ }^{80} \mathrm{Kr}$ Sigma $(\mathrm{mb})$ \\
\hline 13.975 & 233. +/- 9. & 688 \\
\hline 14.415 & $321 .+/-26$. & \\
\hline 14.44 & & 797. \\
\hline 14.685 & & 895. +/- 56. \\
\hline 14.70 & 376. +/- 11 . & \\
\hline
\end{tabular}




\begin{tabular}{|c|c|c|c|c|c|}
\hline$\frac{E_{n}(\mathrm{keV})}{(\mathrm{mb})}$ & Sigma (mb) & $\underline{E}_{n}(\mathrm{keV})$ & Sigma (mb) & $\underline{\mathrm{E}}_{\mathrm{n}}(\mathrm{keV})$ & Sigma \\
\hline 10.0 & $584 .+/-36$. & 47.5 & $395.3+/-6.6$ & 150.0 & $284.5+/-3.4$ \\
\hline 12.0 & $514 .+/-32$. & 50.0 & $311.0+/-5.5$ & 160.0 & $245.7+/-3.6$ \\
\hline 14.0 & 609. +/- 26. & 55.0 & $333.1+/-4.8$ & 170.0 & $171.6+/-3.9$ \\
\hline 16.0 & 489. +/- 21. & 60.0 & $333.9+/-4.6$ & 180.0 & $198.9+/-4.1$ \\
\hline 18.0 & $380 .+/-18$. & 65.0 & $292.2+/-4.5$ & 196.1 & $176.2+/-5.3$ \\
\hline 20.0 & 514. +/- 16. & 70.0 & $253.8+/-4.4$ & 199.8 & $166.3+/-5.4$ \\
\hline 22.0 & 429. +/- 14. & 75.0 & $260.3+/-4.3$ & 203.6 & $132.1+/-5.4$ \\
\hline 24.0 & 413. +/- 13. & 80.0 & $283.7+/-4.2$ & 207.5 & $192.6+/-5.4$ \\
\hline 26.0 & 453. +/- 12. & 85.0 & $221.8+/-4.0$ & 211.6 & $235.0+/-5.3$ \\
\hline 28.0 & 395. +/- 11. & 90.0 & $225.7+/-4.0$ & 215.7 & $214.9+/-5.3$ \\
\hline 30.0 & 417. +/- 9. & 95.0 & $202.4+/-3.9$ & 220.0 & $195.7+/-5.2$ \\
\hline 32.5 & 496. +/- 8 & 100.0 & $200.0+/-3.4$ & 224.4 & $201.3+/-5.0$ \\
\hline 35.0 & 329. +/- 8 . & 110.0 & $187.4+/-3.1$ & 229.0 & $155.1+/-4.8$ \\
\hline 37.5 & 236. +/- 7 & 120.0 & $205.3+/-3.0$ & 233.7 & $183.8+/-4.6$ \\
\hline 40.0 & $311 .+/-7$. & 130.0 & $178.8+/-3.1$ & 238.5 & $216.1+/-4.3$ \\
\hline 42.5 & $368 .+/-7$ & 140.0 & $157.2+/-3.2$ & 243.0 & $272.4+/-4.0$ \\
\hline 45.0 & $324 .+/-7$. & & & & \\
\hline
\end{tabular}

\section{$\underline{\text { YT0488 Set }}$}

The ${ }^{88 \mathrm{~g}, 88 \mathrm{~m} 1,88 \mathrm{~m} 2} \mathrm{Y}(\mathrm{n}, 2 \mathrm{n})^{87 \mathrm{~g}+87 \mathrm{~m}} \mathrm{Y}$ reactions are based on measurements cited in Ref. 13 made on ${ }^{88 \mathrm{~g}} \mathrm{Y}$ at $14.19 \mathrm{MeV}(1140+/-50 \mathrm{mb})$ and at $14.8 \mathrm{MeV}(1180+/-50 \mathrm{mb})$. Note that the data given in Tables 25 and 26 from Ref. 15 are preliminary, and the cross-section set should be revised sometime to use the final data given in Tables 27 and 28 from Ref. 3. $\mathrm{MeV}$.)

Table 22. ${ }^{89} \mathrm{Y}(\mathrm{p}, \mathrm{pn}){ }^{88} \mathrm{Y}$ Reaction. Final results from Ref. 14. (The data extend to 39.6 


\begin{tabular}{|c|c|c|c|c|c|}
\hline$\frac{\mathrm{E}_{\mathrm{p}}(\mathrm{MeV})}{(\mathrm{mb})}$ & $\underline{\text { Sigma }(\mathrm{mb})}$ & $\mathrm{E}_{\mathrm{p}}(\mathrm{MeV})$ & $\underline{\text { Sigma }(\mathrm{mb})}$ & $\underline{\mathrm{E}}_{\mathrm{p}}(\mathrm{MeV})$ & $\underline{\text { Sigma }}$ \\
\hline 12.70 & $\begin{array}{l}0.0123+/- \\
0.0030\end{array}$ & 14.95 & $8.39+/-0.32$ & 19.21 & $115.2+/-3.4$ \\
\hline 13.30 & $0.096+/-0.016$ & 15.41 & $17.87+/-0.55$ & 19.89 & $128.4+/-3.7$ \\
\hline 13.40 & $0.151+/-0.038$ & 15.95 & $31.20+/-0.93$ & 19.92 & $129.8+/-4.2$ \\
\hline 13.80 & $0.272+/-0.108$ & 16.57 & $45.2+/-1.3$ & 20.87 & $146.4+/-4.2$ \\
\hline 14.19 & $1.21+/-0.13$ & 17.39 & $64.8+/-1.9$ & 20.91 & $156.6+/-4.5$ \\
\hline 14.68 & $5.31+/-0.54$ & 18.31 & $85.9+/-2.9$ & 21.44 & $163.9+/-4.9$ \\
\hline
\end{tabular}

$-12-$

Table 23. ${ }^{89} \mathrm{Y}(\mathrm{p}, \mathrm{n}){ }^{89} \mathrm{Zr}$ Reaction. Final results from Ref. 14. The measured cross sections, which are sigma $\left({ }^{89 \mathrm{~g}} \mathrm{Zr}\right)+0.938 * \operatorname{sigma}\left({ }^{89 \mathrm{~m}} \mathrm{Zr}\right)$, have been split into two parts for the $89 \mathrm{~g}$ and $89 \mathrm{~m}$ states using the plot of measured isomer ratio vs. energy in Fig. 4 of Ref. 14. The data extend up to $39.6 \mathrm{MeV}$.

\begin{tabular}{|c|c|c|c|}
\hline$\underline{\mathrm{E}}_{\mathrm{p}}(\mathrm{MeV})$ & Sigma (mb) & ${ }^{89 \mathrm{~g}} \mathrm{Zr}$ Sigma $(\mathrm{mb})$ & ${ }^{89} \mathrm{~m} \mathrm{Zr}$ Sigma $(\mathrm{mb})$ \\
\hline 4.59 & $17.15+/-0.49$ & 7.1 & 10.7 \\
\hline 4.78 & $26.3+/-0.7$ & 10.8 & 16.5 \\
\hline 5.30 & $51.2+/-1.4$ & 21.0 & 32.2 \\
\hline 5.51 & $70.5+/-2.0$ & 28.9 & 44.3 \\
\hline 5.81 & $98.2+/-2.8$ & 40.3 & 61.7 \\
\hline 6.31 & $142.4+/-4.0$ & 58.5 & 89.5 \\
\hline 6.62 & 200. +/- 6 & 82.0 & 126.2 \\
\hline 6.83 & 224. +/- 6 . & 91.7 & 141.3 \\
\hline 7.36 & $330 .+/-9$. & 135.1 & 208.0 \\
\hline 7.90 & 391. +/- 11. & 160.0 & 246 . \\
\hline 8.38 & 467. +/- 13. & 191.1 & 294. \\
\hline 8.98 & 521. +/- 15. & 214 & 327. \\
\hline 9.18 & 573. +/- 16. & 236. & 359. \\
\hline 9.88 & 617. +/- 17. & 256. & 385. \\
\hline 9.94 & 636. +/- 18. & 265. & 396. \\
\hline 10.87 & 675. +/- 19. & 284. & 416. \\
\hline 10.88 & 674. +/- 19. & 284. & 416. \\
\hline 11.97 & 742. +/- 21. & 325. & 444. \\
\hline 12.46 & $751 .+/-21$. & 346. & 432. \\
\hline 12.70 & 749. +/- 21. & 376. & 398. \\
\hline 13.30 & 756. +/- 21. & 417. & 362. \\
\hline 13.40 & $769 .+/-22$. & 428. & 364. \\
\hline 13.80 & 787. +/- 22. & 454. & 355. \\
\hline 14.19 & $765 .+/-22$. & 467. & 317. \\
\hline
\end{tabular}




$\begin{array}{lrrr}14.68 & 700 .+/-20 . & 470 . & 245 . \\ 14.95 & 666 .+/-19 . & 461 . & 219 . \\ 15.41 & 599 .+/-17 . & 424 . & 187 . \\ 15.95 & 536 .+/-15 . & 384 . & 162 . \\ 16.57 & 475 .+/-13 . & 342 . & 142 . \\ 17.39 & 321 .+/-9 . & 233 . & 94 . \\ 18.31 & 241.9+/-7.5 & 176.2 & 70.1 \\ 19.21 & 169.2+/-5.0 & 123.8 & 48.4 \\ 19.89 & 135.3+/-3.8 & 99.1 & 38.6 \\ 19.92 & 140.8+/-4.0 & 103.1 & 40.2 \\ 20.87 & 106.2+/-3.0 & 77.8 & 30.3 \\ 20.91 & 102.0+/-2.9 & 74.7 & 29.1\end{array}$

$-13-$ $\mathrm{MeV}$.

\begin{tabular}{|c|c|c|c|c|c|}
\hline$\frac{\mathrm{E}_{\mathrm{p}}(\mathrm{MeV})}{(\mathrm{mb})}$ & $\underline{\text { Sigma }(\mathrm{mb})}$ & $\underline{\mathrm{E}}_{\mathrm{p}}(\mathrm{MeV})$ & Sigma (mb) & $\mathrm{E}_{\mathrm{p}}(\mathrm{MeV})$ & $\underline{\text { Sigma }}$ \\
\hline 13.30 & $3.62+/-0.14$ & 15.41 & $229.6+/-6.9$ & 19.89 & 726. +/- 21. \\
\hline 13.40 & $6.49+/-0.21$ & 15.95 & $324.8+/-9.3$ & 19.92 & 765. +/- 23. \\
\hline 13.80 & $34.9+/-1.0$ & 16.57 & 418. +/- 12 & 20.87 & 747. +/- 22. \\
\hline 14.19 & $70.0+/-2.0$ & 17.39 & 534. +/- 15. & 20.91 & $775 .+1-22$. \\
\hline 14.68 & $125.2+/-3.6$ & 18.31 & 644. +/- 21. & 21.44 & $765 .+/-23$. \\
\hline 14.95 & $165.9+/-4.8$ & 19.21 & 711. +/- 21. & 22.38 & 784. +/- 24. \\
\hline
\end{tabular}

Table 25. ${ }^{89} \mathrm{Y}(\mathrm{d}, 2 \mathrm{n}){ }^{89} \mathrm{Zr}$ Reaction. Preliminary results from Ref. 15. The measured cross sections, which are sigma $\left({ }^{89 \mathrm{~g}} \mathrm{Zr}\right)+0.938 * \operatorname{sigma}\left({ }^{89 \mathrm{~m}} \mathrm{Zr}\right)$, have been split into two parts for the $89 \mathrm{~g}$ and $89 \mathrm{~m}$ states using a calculated isomer ratio.

\begin{tabular}{|c|c|c|c|}
\hline$\underline{E}_{\mathrm{d}}(\mathrm{MeV})$ & Sigma (mb) & ${ }^{89} \mathrm{Z} Z \mathrm{Zr}$ Sigma $(\mathrm{mb})$ & ${ }^{89 \mathrm{~m}} \mathrm{Zr}$ Sigma $(\mathrm{mb})$ \\
\hline 6.62 & 17.09 & 16.9 & 0.24 \\
\hline 6.67 & 20.10 & 19.7 & 0.48 \\
\hline 7.27 & 77.6 & 68.2 & 10.0 \\
\hline 8.08 & 190.3 & 148.8 & 44.2 \\
\hline 8.40 & 248.8 & 190.3 & 62.4 \\
\hline 9.38 & 400. & 301. & 105.8 \\
\hline 9.89 & 478. & 363. & 123.5 \\
\hline
\end{tabular}




$\begin{array}{lllr}10.49 & 555 . & 426 . & 138.1 \\ 11.37 & 665 . & 522 . & 152.3 \\ 13.08 & 800 . & 651 . & 158.8 \\ 14.64 & 922 . & 771 . & 161.2 \\ 16.29 & 952 . & 814 . & 147.0 \\ 18.14 & 996 . & 879 . & 125.5 \\ 19.84 & 908 . & 824 . & 89.5 \\ 21.10 & 781 . & 723 . & 61.2 \\ 22.78 & 611 . & 583 . & 30.0 \\ 24.36 & 479 . & 468 . & 11.5 \\ 25.74 & 400 . & 392 . & 8.0\end{array}$

\begin{tabular}{|c|c|c|c|c|c|}
\hline$\underline{E}_{d_{d}}(\mathrm{MeV})$ & Sigma (mb) & $\underline{E}_{\mathrm{d}}(\mathrm{MeV})$ & Sigma (mb) & $\underline{E}_{d}(\mathrm{MeV})$ & Sigma (mb) \\
\hline 16.29 & 0.611 & 24.36 & 506. & 32.77 & 696. \\
\hline 18.14 & 20.8 & 25.74 & 601. & 34.23 & 637. \\
\hline 19.84 & 92.4 & 27.57 & 686. & 35.99 & 584. \\
\hline 21.10 & 214.5 & 29.29 & 695. & 37.73 & 510. \\
\hline 22.78 & 371. & 31.13 & 718. & 39.41 & 431. \\
\hline
\end{tabular}

Table 27. ${ }^{89} \mathrm{Y}(\mathrm{d}, 2 \mathrm{n}){ }^{89} \mathrm{Zr}$ Reaction. Final results from Ref. 3. The measured cross sections are sigma $\left({ }^{89 \mathrm{~g}} \mathrm{Zr}\right)+0.938 * \operatorname{sigma}\left({ }^{89 \mathrm{~m}} \mathrm{Zr}\right)$.

\begin{tabular}{|c|c|c|c|c|c|}
\hline $\begin{array}{l}\underline{\mathrm{E}}_{\mathrm{d}}(\mathrm{MeV}) \\
(\mathrm{mb})\end{array}$ & Sigma (mb) & $\underline{\mathrm{E}}_{\mathrm{d}}(\mathrm{MeV})$ & Sigma (mb) & $\underline{\mathrm{E}}_{\mathrm{d}}(\mathrm{MeV})$ & $\underline{\text { Sigma }}$ \\
\hline 6.62 & $15.88+/-0.50$ & 14.64 & 922. +/- 29. & 25.74 & 400. +/- 14 \\
\hline 6.67 & $19.9+/-0.6$ & 15.97 & 982. +/- 31. & 27.57 & $231.4+/-8.1$ \\
\hline 7.27 & $77.3+/-2.3$ & 16.29 & 952. +/- 41. & 29.29 & $262.8+/-8.2$ \\
\hline 8.08 & $190.1+/-5.8$ & 16.67 & $945 .+/-41$. & 31.13 & $225.0+/-8.2$ \\
\hline 8.40 & $248.7+/-7.5$ & 17.50 & $952 .+/-41$. & 32.77 & $197.0+/-6.3$ \\
\hline 9.38 & 400. +/- 12 & 18.14 & 976. +/- 43. & 34.23 & $180.0+/-5.6$ \\
\hline 9.89 & 479. +/- 14. & 19.84 & $908 .+/-39$. & 35.99 & $160.9+/-5.3$ \\
\hline 10.49 & 555. +/- 17. & 21.10 & 781. +/- 34. & 37.73 & $145.6+/-5.9$ \\
\hline 11.37 & $665 .+/-21$. & 22.78 & $611 .+/-27$. & 39.41 & $133.6+/-4.5$ \\
\hline 13.08 & 800. +/- 25 & 24.36 & 479. +/- 15. & & \\
\hline
\end{tabular}

Table 28. ${ }^{89} \mathrm{Y}(\mathrm{d}, 3 \mathrm{n}){ }^{88} \mathrm{Zr}$ Reaction. Final results from Ref. 3. 


\begin{tabular}{|c|c|c|c|c|c|}
\hline$\frac{\underline{E}_{\mathrm{d}}(\mathrm{MeV})}{(\mathrm{mb})}$ & $\underline{\text { Sigma }(\mathrm{mb})}$ & $\underline{\mathrm{E}}_{\mathrm{d}}(\mathrm{MeV})$ & Sigma (mb) & $\underline{\mathrm{E}}_{\mathrm{d}}(\mathrm{MeV})$ & $\underline{\text { Sigma }}$ \\
\hline 15.99 & $0.071+/-0.020$ & 21.10 & $211.4+/-8.6$ & 31.13 & $711 .+/-22$. \\
\hline 16.29 & $0.521+/-0.030$ & 22.78 & 371. +/- 12. & 32.77 & 689. +/- 21. \\
\hline 16.67 & $1.67+/-0.06$ & 24.36 & 499. +/- 15. & 34.23 & 632. +/- 19. \\
\hline 17.50 & $9.18+/-0.35$ & 25.74 & 596. +/- 19. & 35.99 & 579. +/- 18. \\
\hline 18.14 & $20.6+/-0.7$ & 27.57 & 676. +/- 21. & 37.73 & 506. +/- 16 . \\
\hline 19.84 & $91.6+/-2.8$ & 29.29 & 687. +/- 21. & 39.41 & 426. +/- 13. \\
\hline
\end{tabular}

Table 29. ${ }^{89} \mathrm{Y}(\mathrm{n}, 2 \mathrm{n}){ }^{88} \mathrm{Y}$ Reaction. These data are from Ref. 16, and are probably the most accurate measurements available for this reaction.

\begin{tabular}{|c|c|c|c|}
\hline$\underline{E}_{n}(\mathrm{MeV})$ & Sigma (mb) & $\underline{E}_{n}(\mathrm{MeV})$ & Sigma $(\mathrm{mb})$ \\
\hline $\begin{array}{l}13.586+/- \\
0.010\end{array}$ & 698. +/- 9 & $\begin{array}{l}14.476+/- \\
0.012\end{array}$ & $\begin{array}{l}936 .+/- \\
13 .\end{array}$ \\
\hline $\begin{array}{l}14.132+/- \\
0.010\end{array}$ & $\begin{array}{l}868 .+/- \\
11 .\end{array}$ & $\begin{array}{l}14.789+/- \\
0.014\end{array}$ & $\begin{array}{l}1012 .+/- \\
12 .\end{array}$ \\
\hline
\end{tabular}

\section{$\underline{\text { Zr0982 Set }}$}

The ${ }^{88} \operatorname{Zr}(\mathrm{n}, 2 \mathrm{n})^{87}$ reaction is based on a measurement given in Ref. $13(467+/-23 \mathrm{mb}$ at $14.8 \mathrm{MeV})$. The ${ }^{88} \mathrm{Zr}(\mathrm{n}, \mathrm{np})^{87} \mathrm{Y}$ reaction is based on a measurement given in Ref. $13(253+/-$ $25 \mathrm{mb}$ at $14.8 \mathrm{MeV})$. The ${ }^{89} \mathrm{Zr}(\mathrm{n}, 2 \mathrm{n}){ }^{88} \mathrm{Zr}$ reaction is based on measurements given in Ref. 17 at $14.39 \mathrm{MeV}(790+/-40 \mathrm{mb}), 14.47 \mathrm{MeV}(861+/-43 \mathrm{mb})$, and at $15.01 \mathrm{MeV}(878+/-44$ $\mathrm{mb})$.

Table 30. ${ }^{90} \mathrm{Zr}(\mathrm{n}, 2 \mathrm{n}){ }^{89} \mathrm{Zr}$ Reaction. These data are from an evaluation of experimental data given in Ref. 18 (which is by far the best available evaluation). The evaluated cross sections in column $2\left({ }^{89 \mathrm{~g}} \mathrm{Zr}+{ }^{89 \mathrm{~m}} \mathrm{Zr}\right)$ have been split into two parts for the $89 \mathrm{~g}$ and $89 \mathrm{~m}$ states using the calculated isomer ratios from the cross-section set. The indicated neutron energies are the average energies of the bins used to sort the data. 


\begin{tabular}{|c|c|c|c|}
\hline$\underline{\mathrm{E}}_{\mathrm{n}}(\mathrm{MeV})$ & $\underline{\text { Sigma (mb) }}$ & ${ }^{89 \mathrm{~g}} \mathrm{Zr}$ Sigma $(\mathrm{mb})$ & ${ }^{89 \mathrm{~m}} \mathrm{Zr}$ Sigma $(\mathrm{mb})$ \\
\hline 12.30 & $26.3+/-4.0$ & 26.26 & 0.0 \\
\hline 12.55 & $82.3+/-8.8$ & 82.35 & 0.0 \\
\hline 13.00 & $247.6+/-12.5$ & 245.1 & 2.52 \\
\hline 13.40 & $392.0+/-6.8$ & 368.5 & 23.5 \\
\hline 13.60 & $461.4+/-8.8$ & 425.0 & 36.4 \\
\hline 13.80 & $535.5+/-7.0$ & 482.8 & 52.7 \\
\hline 14.00 & $591.3+/-6.4$ & 521.0 & 70.3 \\
\hline 14.20 & $652.3+/-8.9$ & 561.5 & 90.8 \\
\hline 14.40 & $717.8+/-6.8$ & 607.7 & 110.1 \\
\hline 14.60 & $763.8+/-10.4$ & 639.1 & 124.7 \\
\hline 14.80 & $805.1+/-8.0$ & 665.8 & 139.3 \\
\hline 15.15 & $901 .+/-22$ & 732.0 & 169. \\
\hline 16.00 & 1017. +/- 26 & 805. & 212. \\
\hline 17.15 & $1125 .+/-32$ & 885. & 240. \\
\hline 18.35 & 1189. +/- 29. & 962. & 227. \\
\hline 20.00 & 1200. +/- 30 & 1000. & 200. \\
\hline
\end{tabular}

\section{II0391 Set}

Note that the data in Tables 31 and 32 are as used in the cross-section set. However, the cross sections should be lowered sometime by the factor, 0.984 , to correct to the photon abundances used in Test program measurements.

$-16-$

\begin{tabular}{|c|c|c|c|c|c|}
\hline$\frac{\mathrm{E}_{\mathrm{p}}(\mathrm{MeV})}{(\mathrm{mb})}$ & Sigma (mb) & $\underline{\mathrm{E}}_{\mathrm{p}}(\mathrm{MeV})$ & $\underline{\text { Sigma (mb) }}$ & $\underline{\mathrm{E}}_{\mathrm{p}}(\mathrm{MeV})$ & $\underline{\text { Sigma }}$ \\
\hline 3.35 & $0.126+/-0.006$ & 8.02 & 216. $+/-7$. & 12.19 & $261 .+/-8$ \\
\hline 3.80 & $0.482+/-0.018$ & 8.10 & 251. +/- 8. & 12.98 & $196.0+/-5.9$ \\
\hline 4.53 & $3.43+/-0.11$ & 8.68 & 330. +/- 10. & 13.02 & $191.1+/-6.0$ \\
\hline 5.11 & $7.90+/-0.26$ & 8.90 & $384 .+/-12$ & 13.82 & $141.1+/-4.3$ \\
\hline 5.70 & $28.4+/-0.9$ & 9.09 & 394. +/- 12. & 14.25 & $118.3+/-3.6$ \\
\hline 6.12 & $48.6+/-1.5$ & 9.65 & 435. +/- 13. & 14.59 & $101.5+/-3.1$ \\
\hline 6.67 & $87.4+/-2.6$ & 10.41 & 419. +/- 13. & 15.53 & $81.0+/-2.4$ \\
\hline 7.12 & $108.4+/-3.3$ & 11.30 & 337. +/- 10 . & 16.84 & $57.9+/-1.8$ \\
\hline 7.63 & $195.4+/-5.9$ & & & & \\
\hline
\end{tabular}




\begin{tabular}{|c|c|c|c|c|c|}
\hline$\frac{\mathrm{E}_{\mathrm{d}}(\mathrm{MeV})}{(\mathrm{mb})}$ & $\underline{\operatorname{Sigma}(\mathrm{mb})}$ & $\underline{\mathrm{E}}_{\mathrm{d}}(\mathrm{MeV})$ & $\underline{\text { Sigma }(\mathrm{mb})}$ & $\underline{\mathrm{E}}_{\mathrm{d}}(\mathrm{MeV})$ & $\underline{\text { Sigma }}$ \\
\hline 4.19 & $0.020+/-0.002$ & 7.33 & $147.8+/-4.5$ & 10.98 & 715. +/- 22 \\
\hline 4.21 & $0.026+/-0.004$ & 7.53 & $163.0+/-4.9$ & 11.67 & 779. +/- 23. \\
\hline 4.54 & $0.256+/-0.057$ & 8.10 & 250. +/- 8 & 12.73 & 853. +/- 26. \\
\hline 4.86 & $0.92+/-0.03$ & 8.39 & 322. +/- 10. & 13.13 & 882. +/- 27. \\
\hline 5.24 & $3.82+/-0.15$ & 8.56 & $361 .+/-11$. & 14.38 & 889. +/- 27 \\
\hline 5.54 & $9.67+/-0.31$ & 9.51 & 479. +/- 14 & 14.55 & $883 .+/-27$. \\
\hline 6.40 & $44.5+/-1.4$ & 9.94 & $581 .+/-18$ & 15.73 & 772. +/- 23. \\
\hline 6.75 & $71.5+/-2.2$ & 10.43 & 616. +/- 19. & 16.79 & 677. +/- 20. \\
\hline
\end{tabular}

Table 33. ${ }^{127} \mathrm{I}(\mathrm{n}, \mathrm{g}){ }^{128} \mathrm{I}$ Reaction. Final results from Ref. 19.

\begin{tabular}{|c|c|c|c|c|c|}
\hline$\underline{E}_{n}(\mathrm{keV})$ & $\underline{\operatorname{Sigma}(\mathrm{mb})}$ & $\underline{E}_{n}(\mathrm{keV})$ & $\underline{\operatorname{Sigma}(\mathrm{mb})}$ & $\underline{\mathrm{E}}_{\mathrm{n}}(\mathrm{keV})$ & $\underline{\operatorname{Sigma}(\mathrm{mb})}$ \\
\hline 3.5 & 1845 & 175 & 213 & 1050. & 73.6 \\
\hline 5.0 & 1522. & 250. & 170. & 1150. & 71.0 \\
\hline 7.0 & 1303. & 350. & 137. & 1250. & 72.3 \\
\hline 9.0 & 1221. & 450. & 123. & 1350. & 65.8 \\
\hline 12.5 & 1018. & 550. & 116. & 1450. & 66.7 \\
\hline 17.5 & 858. & 650. & 106. & 1550. & 68.2 \\
\hline 25.0 & 751. & 713. & 98.5 & 1650. & 66.5 \\
\hline 35.0 & 622. & 738. & 95.8 & 1750. & 56.8 \\
\hline 50.0 & 518. & 763. & 92.2 & 1850. & 56.4 \\
\hline 70.0 & 400. & 788. & 80.3 & 1950. & 57.2 \\
\hline 90.0 & 322. & 850. & 78.2 & 2050. & 55.0 \\
\hline 125. & 260. & 950. & 73.5 & 2150 . & 52.3 \\
\hline
\end{tabular}

$-17-$

Table 34. ${ }^{127} \mathrm{I}(\mathrm{n}, \mathrm{g}){ }^{128} \mathrm{I}$ Reaction. Final results from Ref. 20 . The data were visually taken from a plot of experimental data, replotted, and read from that plot.

\begin{tabular}{|c|c|c|c|c|c|}
\hline$\underline{E}_{\mathrm{n}}(\mathrm{MeV})$ & Sigma (mb) & $\underline{E}_{\mathrm{n}}(\mathrm{MeV})$ & $\underline{\operatorname{Sigma}(\mathrm{mb})}$ & $\underline{\mathrm{E}}_{\mathrm{n}}(\mathrm{MeV})$ & $\underline{\text { Sigma }(\mathrm{mb})}$ \\
\hline 2.5 & 32.7 & 6.0 & 7.6 & 12.0 & 3.15 \\
\hline 3.0 & 22.1 & 7.0 & 6.16 & 14.0 & 2.62 \\
\hline
\end{tabular}




$\begin{array}{rrrrrr}3.5 & 17.0 & 8.0 & 5.20 & 16.0 & 2.25 \\ 4.0 & 13.7 & 9.0 & 4.46 & 20.0 & 1.74 \\ 5.0 & 9.8 & 10.0 & 3.92 & & \end{array}$

\section{Eu0988 Set}

The ${ }^{151} \mathrm{Eu}(\mathrm{n}, 2 \mathrm{n}){ }^{150} \mathrm{Eu}$ and ${ }^{153} \mathrm{Eu}(\mathrm{n}, 2 \mathrm{n}){ }^{152} \mathrm{Eu}$ reactions are based on an evaluation in Ref. 24. Cross sections for the ${ }^{151} \mathrm{Eu}$ reaction were scaled down by the factor, 0.911, to match the measured total $(n, 2 n)$ cross section, $1.798 \mathrm{~b}$ at $14.8 \mathrm{MeV}$. Cross sections for the ${ }^{153} \mathrm{Eu}$ reaction are in agreement with the measured value, $2.05+/-0.14 \mathrm{~b}$ at $14.7 \mathrm{MeV}^{25}$

Table 35. ${ }^{151} \mathrm{Eu}(\mathrm{p}, \mathrm{n}){ }^{151} \mathrm{Gd}$ Reaction. Preliminary results from Refs. 16 and 17 . The results in columns 2 and 5 were scaled up by the factor, 1.216, to correct to the photon abundances in use for Test Program measurements, and are given in columns 3 and 6.

\begin{tabular}{|c|c|c|c|c|c|}
\hline$\frac{\mathrm{E}_{\mathrm{p}}(\mathrm{MeV})}{(\mathrm{mb})}$ & Sigma (mb) & Scaled (mb) & $\underline{\mathrm{E}}_{\mathrm{p}}(\mathrm{MeV})$ & Sigma (mb) & $\underline{\text { Scaled }}$ \\
\hline 5.49 & 6.47 & 7.87 & 8.21 & 128.2 & 155.9 \\
\hline 5.70 & 7.47 & 9.08 & 8.47 & 149.3 & 181.5 \\
\hline 6.02 & 11.97 & 14.56 & 9.29 & 187.3 & 227.8 \\
\hline 6.10 & 13.97 & 16.99 & 9.52 & 186.5 & 226.8 \\
\hline 6.38 & 19.51 & 23.72 & 10.09 & 196.5 & 238.9 \\
\hline 6.63 & 30.7 & 37.4 & 10.29 & 189.2 & 230.1 \\
\hline 6.92 & 42.7 & 51.9 & 10.48 & 180.2 & 219.1 \\
\hline 7.16 & 55.6 & 67.6 & 10.95 & 184.4 & 224.2 \\
\hline 7.85 & 99.4 & 120.9 & 11.58 & 149.0 & 181.1 \\
\hline
\end{tabular}

$-18-$

Table 36. ${ }^{151} \mathrm{Eu}(\mathrm{d}, 2 \mathrm{n}){ }^{151} \mathrm{Gd}$ Reaction. Preliminary results from Refs. 15 and 17 . The results in columns 2 and 5 were scaled up by the factor, 1.216, to correct to the photon abundances in use for Test Program measurements, and are given in columns 3 and 6. 


\begin{tabular}{|c|c|c|c|c|c|}
\hline$\underline{E}_{\mathrm{d}}(\mathrm{MeV})$ & Sigma (mb) & Scaled (mb) & $\underline{\mathrm{E}}_{\mathrm{d}}(\mathrm{MeV})$ & Sigma (mb) & Scaled (mb) \\
\hline 4.84 & 0.178 & 0.216 & 8.03 & 133.2 & 162.0 \\
\hline 5.88 & 5.29 & 6.43 & 8.24 & 155.7 & 189.3 \\
\hline 5.91 & 4.98 & 6.06 & 8.89 & 241.9 & 294.2 \\
\hline 6.37 & 13.98 & 17.00 & 9.37 & 295.4 & 359. \\
\hline 6.74 & 25.78 & 31.35 & 9.81 & 326. & 397. \\
\hline 7.22 & 68.3 & 83.1 & 10.22 & 392. & 476. \\
\hline 7.60 & 81.6 & 99.2 & 10.35 & 419. & 510. \\
\hline 7.99 & 118.8 & 144.5 & 11.18 & 528. & 642. \\
\hline
\end{tabular}

Table 37. ${ }^{153} \mathrm{Eu}(\mathrm{p}, \mathrm{n}){ }^{153} \mathrm{Gd}$ Reaction. Preliminary results from Refs. 16 and 17 . The results in columns 2 and 5 were scaled down by the factor, 0.861 , to correct to the photon abundances in use for Test Program measurements, and are given in columns 3 and 6.

\begin{tabular}{|c|c|c|c|c|c|}
\hline$\frac{\mathrm{E}_{\mathrm{p}}(\mathrm{MeV})}{(\mathrm{mb})}$ & Sigma (mb) & $\underline{\text { Scaled (mb) }}$ & $\mathrm{E}_{\mathrm{p}}(\mathrm{MeV})$ & Sigma (mb) & $\underline{\text { Scaled }}$ \\
\hline 5.49 & 8.70 & 7.49 & 8.21 & 160.1 & 137.9 \\
\hline 5.70 & 10.18 & 8.77 & 8.47 & 182.6 & 157.3 \\
\hline 6.02 & 16.37 & 14.10 & 9.29 & 198.3 & 170.8 \\
\hline 6.10 & 19.06 & 16.42 & 9.52 & 208.0 & 179.2 \\
\hline 6.38 & 25.50 & 21.97 & 10.09 & 214.5 & 184.8 \\
\hline 6.63 & 41.3 & 35.6 & 10.29 & 194.6 & 167.6 \\
\hline 6.92 & 57.5 & 49.5 & 10.48 & 191.1 & 164.6 \\
\hline 7.16 & 74.0 & 63.7 & 10.95 & 183.1 & 157.7 \\
\hline 7.85 & 131.0 & 112.8 & 11.58 & 163.2 & 140.6 \\
\hline
\end{tabular}

Table 38. ${ }^{153} \mathrm{Eu}(\mathrm{d}, 2 \mathrm{n}){ }^{153} \mathrm{Gd}$ Reaction. Preliminary results from Refs. 15 and 17 . The results in columns 2 and 5 were scaled down by the factor, 0.861 , to correct to the photon abundances in use for Test Program measurements, and are given in columns 3 and 6.

\begin{tabular}{|c|c|c|c|c|c|}
\hline$\underline{E}_{\mathrm{d}}(\mathrm{MeV})$ & Sigma (mb) & Scaled (mb) & $\underline{\mathrm{E}}_{\mathrm{d}}(\mathrm{MeV})$ & Sigma (mb) & Scaled (mb) \\
\hline 4.84 & 0.308 & 0.265 & 8.03 & 177.9 & 153.2 \\
\hline 5.88 & 7.93 & 6.83 & 8.24 & 208.7 & 179.8 \\
\hline 5.91 & 7.31 & 6.30 & 8.89 & 319.9 & 275.6 \\
\hline 6.37 & 20.18 & 17.38 & 9.37 & 379. & 326.5 \\
\hline 6.74 & 36.6 & 31.5 & 9.81 & 426. & 367. \\
\hline 7.22 & 92.7 & 79.9 & 10.22 & 511. & 440. \\
\hline 7.60 & 112.6 & 97.0 & 10.35 & 540. & 465. \\
\hline 7.99 & 160.2 & 138.0 & 11.18 & 670. & 577. \\
\hline
\end{tabular}


Table 39. ${ }^{151} \mathrm{Eu}(\mathrm{p}, \mathrm{n}){ }^{151} \mathrm{Gd}$ Reaction. Final results from Ref. 3. The results in columns 2 and 5 were scaled up by the factor, 1.216 , to correct to the photon abundances in use for Test Program measurements, and are given in columns 3 and 6. (The data extend up to 35.5 $\mathrm{MeV}$.)

\begin{tabular}{|c|c|c|c|c|c|}
\hline$\frac{\underline{\mathrm{E}}_{\mathrm{p}}(\mathrm{MeV})}{(\mathrm{mb})}$ & $\underline{\text { Sigma (mb) }}$ & $\underline{\text { Scaled }(\mathrm{mb})}$ & $\underline{\mathrm{E}}_{\mathrm{p}}(\mathrm{MeV})$ & $\underline{\operatorname{Sigma}(\mathrm{mb})}$ & $\underline{\text { Scaled }}$ \\
\hline 4.72 & $1.62+/-0.06$ & 1.97 & 12.03 & $131.0+/-4.0$ & 159.3 \\
\hline 5.50 & $6.44+/-0.20$ & 7.83 & 13.28 & $93.5+/-3.1$ & 113.7 \\
\hline 5.71 & $7.40+/-0.23$ & 9.00 & 13.61 & $81.8+/-2.5$ & 99.5 \\
\hline 5.91 & $11.54+/-0.36$ & 14.03 & 13.66 & $84.9+/-2.5$ & 103.2 \\
\hline 6.03 & $11.98+/-0.37$ & 14.57 & 14.44 & $64.4+/-2.0$ & 78.3 \\
\hline 6.12 & $15.5+/-0.5$ & 18.8 & 14.62 & $61.9+/-1.9$ & 75.3 \\
\hline 6.38 & $20.3+/-0.6$ & 24.7 & 14.81 & $56.7+/-1.7$ & 68.9 \\
\hline 6.42 & $24.9+/-0.9$ & 30.3 & 15.05 & $52.2+/-1.6$ & 63.5 \\
\hline 6.63 & $30.5+/-1.0$ & 37.1 & 15.71 & $45.1+/-1.4$ & 54.8 \\
\hline 6.93 & $42.7+/-1.5$ & 51.9 & 15.91 & $42.2+/-1.3$ & 51.3 \\
\hline 7.17 & $54.8+/-1.8$ & 66.6 & 16.38 & $38.0+/-1.2$ & 46.2 \\
\hline 7.44 & $66.4+/-2.1$ & 80.7 & 16.78 & $37.6+/-1.2$ & 45.7 \\
\hline 7.85 & $98.9+/-3.0$ & 120.3 & 16.89 & $35.6+/-1.1$ & 43.3 \\
\hline 8.20 & $128.2+/-4.0$ & 155.9 & 17.79 & $34.0+/-$ & 41.3 \\
\hline 8.48 & $151.0+/-4.8$ & 183.6 & 17.93 & $33.7+/-$ & 41.0 \\
\hline 8.82 & $168.4+/-5.4$ & 204.8 & 18.15 & $33.1+/-$ & 40.3 \\
\hline 9.29 & $190.9+/-6.1$ & 232.1 & 18.77 & $31.3+/-$ & 38.1 \\
\hline 10.10 & $195.9+/-6.1$ & 238.2 & 19.30 & $30.2+/-$ & 36.7 \\
\hline 10.30 & $192.5+/-6.0$ & 234.1 & 19.70 & $29.4+/-$ & 35.8 \\
\hline 10.48 & $186.2+/-5.8$ & 226.4 & 19.93 & $29.1+/-$ & 35.4 \\
\hline 10.65 & $185.4+/-5.7$ & 225.5 & 20.42 & $28.3+/-$ & 34.4 \\
\hline 10.95 & $169.1+/-5.5$ & 205.6 & 21.05 & $27.8+/-$ & 33.8 \\
\hline 11.58 & $152.8+/-5.0$ & 185.8 & 21.50 & $26.8+/-$ & 32.6 \\
\hline
\end{tabular}


Table 40. ${ }^{151} \mathrm{Eu}(\mathrm{d}, 2 \mathrm{n}){ }^{151} \mathrm{Gd}$ Reaction. Final results from Ref. 3. The results in columns 2 and 5 were scaled up by the factor, 1.216 , to correct to the photon abundances in use for Test Program measurements, and are given in columns 3 and 6. (The data extend up to 33.3 $\mathrm{MeV}$.)

\begin{tabular}{|c|c|c|c|c|c|}
\hline$\frac{\mathrm{E}_{\mathrm{d}}(\mathrm{MeV})}{(\mathrm{mb})}$ & $\underline{\operatorname{Sigma}(\mathrm{mb})}$ & $\underline{\text { Scaled (mb) }}$ & $\underline{\mathrm{E}}_{\mathrm{d}}(\mathrm{MeV})$ & $\underline{\operatorname{Sigma}(\mathrm{mb})}$ & $\underline{\text { Scaled }}$ \\
\hline 4.86 & $0.26+/-0.03$ & 0.32 & 12.32 & 589. +/- 18 & 716. \\
\hline 5.90 & $5.46+/-0.18$ & 6.64 & 12.72 & 624. +/- 19. & 759. \\
\hline 5.92 & $5.10+/-0.18$ & 6.20 & 13.06 & 622. +/- 19. & 754. \\
\hline 6.39 & $14.19+/-0.46$ & 17.26 & 14.14 & 624. +/- 19. & 759. \\
\hline 6.75 & $26.04+/-0.80$ & 31.66 & 14.19 & 621. +/- 19. & 756. \\
\hline 7.26 & $68.8+/-2.1$ & 83.6 & 14.40 & 588. +/- 18. & 715. \\
\hline 7.61 & $81.1+/-2.5$ & 98.6 & 15.46 & 549. +/- 17. & 667. \\
\hline 8.03 & $135.3+/-4.1$ & 164.5 & 15.64 & 531. +/- 16. & 646. \\
\hline 8.28 & $154.9+/-4.7$ & 188.4 & 16.69 & 459. +/- 15. & 558. \\
\hline 8.91 & $241.7+/-7.3$ & 293.9 & 16.80 & 437. +/- 13. & 531. \\
\hline 9.38 & $293.0+/-8.8$ & 356.3 & 17.94 & $361 .+/-12$. & 439. \\
\hline 9.81 & 360. +/- 11 & 438. & 19.06 & 298. +/- 9 & 362. \\
\hline 10.22 & 392. +/- 12. & 476. & 19.34 & 284. +/- 9 & 345. \\
\hline 10.35 & 419. +/- 13. & 510. & 20.87 & 231. +/- 7 & 281. \\
\hline 11.18 & 522. +/- 16. & 634. & 22.30 & 187. +/- & 227. \\
\hline 11.35 & 518. +/- 17 . & 629. & 23.64 & 163. +/- & 198. \\
\hline
\end{tabular}

Table 41. ${ }^{153} \mathrm{Eu}(\mathrm{p}, \mathrm{n}){ }^{153} \mathrm{Gd}$ Reaction. Final results from Ref. 3. The results in columns 2 and 5 were scaled down by the factor, 0.861 , to correct to the photon abundances in use for Test Program measurements, and are given in columns 3 and 6. (The data extend up to 35.5 $\mathrm{MeV}$.)

\begin{tabular}{|c|c|c|c|c|c|}
\hline$\frac{\mathrm{E}_{\mathrm{p}}(\mathrm{MeV})}{(\mathrm{mb})}$ & $\underline{\text { Sigma (mb) }}$ & $\underline{\text { Scaled (mb) }}$ & $\underline{\mathrm{E}}_{\mathrm{p}}(\mathrm{MeV})$ & $\underline{\text { Sigma }(\mathrm{mb})}$ & $\underline{\text { Scaled }}$ \\
\hline 4.72 & $2.35+/-0.07$ & 2.02 & 12.03 & $148.4+/-4.5$ & 127.7 \\
\hline 5.50 & $8.92+/-0.27$ & 7.68 & 13.28 & $101.0+/-3.1$ & 87.0 \\
\hline 5.71 & $10.20+/-0.31$ & 8.78 & 13.61 & $90.6+/-2.8$ & 78 \\
\hline 5.91 & $16.30+/-0.49$ & 14.03 & 13.66 & $90.9+/-2.7$ & 78.3 \\
\hline 6.03 & $16.56+/-0.51$ & 14.26 & 14.44 & $71.5+/-2.2$ & 61.6 \\
\hline 6.12 & $21.1+/-0.6$ & 18.2 & 14.62 & $68.3+/-2.1$ & 58.8 \\
\hline 6.38 & $27.9+/-0.9$ & 24.0 & 14.81 & $62.6+/-1.9$ & 53.9 \\
\hline 6.42 & $34.5+/-1.0$ & 29.7 & 15.05 & $58.6+/-1.8$ & 50. \\
\hline 6.63 & $41.8+/-1.3$ & 36.0 & 15.71 & $51.1+/-1.5$ & 44. \\
\hline 6.93 & $58.2+/-1.7$ & 50.1 & 15.91 & $47.7+/-1.4$ & 41 \\
\hline 7.17 & $74.3+/-2.2$ & 64.0 & 16.38 & $45.1+/-1.4$ & 38.8 \\
\hline
\end{tabular}




\begin{tabular}{rrrrrr}
7.44 & $92.6+/-2.8$ & 79.7 & 16.78 & $42.9+/-1.3$ & 36.9 \\
7.85 & $129.7+/-3.9$ & 111.7 & 16.89 & $40.2+/-1.2$ & 34.6 \\
8.20 & $160.1+/-4.8$ & 137.8 & 17.79 & $38.7+/-1.3$ & 33.3 \\
8.48 & $185.1+/-5.6$ & 159.4 & 17.93 & $36.3+/-1.2$ & 31.3 \\
8.82 & $202.0+/-6.9$ & 173.9 & 18.15 & $35.3+/-1.2$ & 30.4 \\
\multicolumn{2}{l}{ (continued on next page) } & & & &
\end{tabular}

\section{$-21-$}

Table 41, continued.

\begin{tabular}{|c|c|c|c|c|c|}
\hline$\underline{\mathrm{E}}_{\mathrm{p}}(\mathrm{MeV})$ & Sigma (mb) & Scaled (mb) & $\underline{\mathrm{E}}_{\mathrm{p}}(\mathrm{MeV})$ & Sigma (mb) & Scaled (mb) \\
\hline 9.29 & $215.4+/-6.5$ & 185.5 & 18.77 & $35.2+/-1.1$ & 30.3 \\
\hline 10.10 & $212.0+/-6.4$ & 182.5 & 19.30 & $34.8+/-1.1$ & 30.0 \\
\hline 10.30 & $208.1+/-6.2$ & 179.2 & 19.70 & $33.9+/-1.1$ & 29.2 \\
\hline 10.48 & $191.1+/-5.8$ & 164.5 & 19.93 & $32.2+/-1.1$ & 27.7 \\
\hline 10.65 & $200.2+/-6.0$ & 172.4 & 20.42 & $32.3+/-1.1$ & 27.8 \\
\hline 10.95 & $187.1+/-5.8$ & 161.1 & 21.05 & $31.3+/-1.7$ & 26.9 \\
\hline 11.58 & $170.3+/-5.3$ & 146.6 & 21.50 & $30.4+/-1.1$ & 26.2 \\
\hline
\end{tabular}

Table 42. ${ }^{153} \mathrm{Eu}(\mathrm{d}, 2 \mathrm{n}){ }^{153} \mathrm{Gd}$ Reaction. Final results from Ref. 3. The results in columns 2 and 5 were scaled down by the factor, 0.861 , to correct to the photon abundances in use for Test Program measurements, and are given in columns 3 and 6. (The data extend up to 33.3 $\mathrm{MeV}$.)

\begin{tabular}{|c|c|c|c|c|c|}
\hline$\frac{\mathrm{E}_{\mathrm{d}}(\mathrm{MeV})}{(\mathrm{mb})}$ & Sigma (mb) & Scaled (mb) & $\underline{\mathrm{E}}_{\mathrm{d}}(\mathrm{MeV})$ & Sigma (mb) & Scaled \\
\hline 4.86 & $0.39+/-0.02$ & 0.34 & 12.32 & 754. +/- 23. & 649. \\
\hline 5.90 & $8.01+/-0.25$ & 6.90 & 12.72 & 766. +/- 23. & 660. \\
\hline 5.92 & $7.38+/-0.23$ & 6.35 & 13.06 & 812. +/- 24. & 699. \\
\hline 6.39 & $20.35+/-0.63$ & 17.52 & 14.14 & 728. +/- 22. & 627. \\
\hline 6.75 & $36.6+/-1.1$ & 31.5 & 14.19 & 749. +/- 23. & 645. \\
\hline 7.26 & $93.6+/-2.8$ & 80.6 & 14.40 & 694. +/- 21. & 598. \\
\hline 7.61 & $111.6+/-3.4$ & 96.1 & 15.46 & 619. +/- 19 . & 533. \\
\hline 8.03 & $180.8+/-5.5$ & 155.7 & 15.64 & 609. +/- 18. & 524. \\
\hline 8.28 & $205.7+/-6.2$ & 177.1 & 16.69 & 496. +/- 15. & 427. \\
\hline 8.91 & 318. +/- 10 & 273.7 & 16.80 & 485. +/- 15. & 418. \\
\hline 9.38 & 380. +/- 11. & 327. & 17.94 & 398. +/- 12 . & 343. \\
\hline 9.81 & 470. +/- 14 & 405. & 19.06 & 327. +/- 10 . & 282. \\
\hline 10.22 & 511. +/- 15 & 440. & 19.34 & 307. +/- 9. & 264. \\
\hline 10.35 & 545. +/- 16. & 469. & 20.87 & $248 .+/-7$ & 214. \\
\hline 11.18 & 669. +/- 20. & 576. & 22.30 & 200. +/- 6 & 172. \\
\hline 11.35 & 694. +/- 21. & 597. & 23.64 & 175. +/- 5 & 151. \\
\hline
\end{tabular}


Table 43. ${ }^{151} \mathrm{Eu}(\mathrm{n}, \mathrm{g}){ }^{152} \mathrm{Eu}$ Reaction. Final results from Ref. 23. The indicated neutron energies are the average energies of the bins used to sort the data.

\begin{tabular}{|c|c|c|c|c|c|}
\hline$\underline{E}_{n}(\mathrm{MeV})$ & Sigma (mb) & $\underline{\mathrm{E}}_{\mathrm{n}}(\mathrm{MeV})$ & $\underline{\operatorname{Sigma}(\mathrm{mb})}$ & $\underline{\mathrm{E}}_{\mathrm{n}}(\mathrm{MeV})$ & Sigma (mb) \\
\hline 0.0035 & 14620 & 0.175 & 1412 & 1.05 & 377. \\
\hline 0.0050 & 11570. & 0.25 & 1224. & 1.15 & 338. \\
\hline 0.0070 & 8990. & 0.35 & 884. & 1.25 & 319. \\
\hline 0.0090 & 7520. & 0.45 & 711. & 1.35 & 287. \\
\hline 0.0125 & 6106. & 0.55 & 637. & 1.45 & 262. \\
\hline 0.0175 & 4805. & 0.65 & 547. & 1.55 & 247. \\
\hline 0.025 & 3722. & 0.7125 & 503. & 1.65 & 232. \\
\hline 0.035 & 2851. & 0.7375 & 502. & 1.75 & 227. \\
\hline 0.050 & 2320. & 0.7625 & 482. & 1.85 & 196. \\
\hline 0.070 & 1939. & 0.7875 & 473. & 1.95 & 199. \\
\hline 0.090 & 1717. & 0.85 & 439. & 2.05 & 164. \\
\hline 0.125 & 1551. & 0.95 & 399. & 2.15 & 155. \\
\hline
\end{tabular}

Table 44. ${ }^{153} \mathrm{Eu}(\mathrm{n}, \mathrm{g}){ }^{154} \mathrm{Eu}$ Reaction. Final results from Ref. 23. The indicated neutron energies are the average energies of the bins used to sort the data.

\begin{tabular}{|c|c|c|c|c|c|}
\hline$\underline{E}_{n}(\mathrm{MeV})$ & Sigma (mb) & $\underline{\mathrm{E}}_{\mathrm{n}}(\mathrm{MeV})$ & $\underline{\text { Sigma }(\mathrm{mb})}$ & $\underline{\mathrm{E}}_{\mathrm{n}}(\mathrm{MeV})$ & $\underline{\operatorname{Sigma}(\mathrm{mb})}$ \\
\hline 0.0035 & 8320 & 0.175 & 916. & 1.05 & 250 \\
\hline 0.0050 & 6480. & 0.25 & 683. & 1.15 & 229. \\
\hline 0.0070 & 5210. & 0.35 & 500. & 1.25 & 214. \\
\hline 0.0090 & 4597. & 0.45 & 429. & 1.35 & 202. \\
\hline 0.0125 & 3837. & 0.55 & 411. & 1.45 & 188. \\
\hline 0.0175 & 3168. & 0.65 & 378. & 1.55 & 183. \\
\hline 0.025 & 2597. & 0.7125 & 349. & 1.65 & 174. \\
\hline 0.035 & 2219. & 0.7375 & 336. & 1.75 & 163. \\
\hline 0.050 & 1914. & 0.7625 & 324. & 1.85 & 151. \\
\hline 0.070 & 1668. & 0.7875 & 326. & 1.95 & 156. \\
\hline
\end{tabular}




$\begin{array}{llllll}0.090 & 1491 . & 0.85 & 299 . & 2.05 & 128 . \\ 0.125 & 1189 . & 0.95 & 284 . & 2.15 & 126 .\end{array}$

Table 45. ${ }^{152} \mathrm{Gd}(\mathrm{n}, \mathrm{g}){ }^{153} \mathrm{Gd}$ Reaction. Final results from Ref. 26. The indicated neutron energies are the average energies of the bins used to sort the data.

\begin{tabular}{|c|c|c|c|c|c|}
\hline$\frac{\mathrm{E}_{\mathrm{n}}(\mathrm{keV})}{(\mathrm{mb})}$ & $\underline{\text { Sigma (mb) }}$ & $\underline{\mathrm{E}}_{\mathrm{n}}(\mathrm{keV})$ & $\underline{\text { Sigma }(\mathrm{mb})}$ & $\underline{E}_{\mathrm{n}}(\mathrm{keV})$ & $\underline{\text { Sigma }}$ \\
\hline 3.5 & $3400 .+/-100$ & 25. & 1008. +/- 29 & 175. & 538. +/- 18 . \\
\hline 5.0 & 2730. +/- 80 & 35. & $905 .+/-26$. & 250. & 531. +/- 18. \\
\hline 7.0 & 2246. +/- 66. & 50. & $784 .+/-23$. & 350. & 461. +/- 16. \\
\hline 9.0 & 1785. +/- 52. & 70. & 658. +/- 20. & 450. & 398. +/- 14. \\
\hline 12.5 & 1426. +/- 42 & 90. & 622. +/- 19. & 550. & 393. +/- 16. \\
\hline 17.5 & 1186. +/- 35. & 125. & 569. +/- 18 & 650. & 390. +/- 16 . \\
\hline
\end{tabular}

Table 46. ${ }^{154} \mathrm{Gd}(\mathrm{n}, \mathrm{g}){ }^{155} \mathrm{Gd}$ Reaction. Final results from Ref. 26. The indicated neutron energies are the average energies of the bins used to sort the data.

\begin{tabular}{|c|c|c|c|c|c|}
\hline$\frac{E_{n}(\mathrm{keV})}{(\mathrm{mb})}$ & Sigma (mb) & $\underline{E}_{n}(\mathrm{keV})$ & Sigma (mb) & $\underline{E}_{n}(\mathrm{keV})$ & Sigma \\
\hline 3.5 & 2818. +/- 82 . & 25. & 914. +/- 27. & 175. & 393. +/- 13. \\
\hline 5.0 & 2196. +/- 64 & 35. & 771. +/- 23. & 250. & 337. +/- 11. \\
\hline 7.0 & 1692. +/- 49 . & 50. & 682. +/- 20. & 350. & 302. +/- 10. \\
\hline 9.0 & 1640. +/- 48 & 70. & 591. +/- 18. & 450. & 292. +/- 10. \\
\hline 12.5 & 1239. +/- 36 & 90. & $560 .+/-17$. & & \\
\hline 17.5 & 1044. +/- 30 & 125. & 486. +/- 16. & & \\
\hline
\end{tabular}

\section{Lu0284 Set}


The reactions in this set are all based on calculated excitation functions. The cross section for the ${ }^{173} \mathrm{Lu}(\mathrm{n}, 2 \mathrm{n}){ }^{172} \mathrm{Lu}$ reaction is within $1 \%$ of the measured value, $2.05+/-0.10 \mathrm{~b}$ at $14.8 \mathrm{MeV},{ }^{27}$

while that for the ${ }^{175} \mathrm{Lu}(\mathrm{n}, 2 \mathrm{n}){ }^{174 t} \mathrm{Lu}$ reaction is within $5 \%$ of evaluated data near $14 \mathrm{MeV}$.

\section{Bi0389 Set}

The reactions in this set are all based on calculated excitation functions. The cross section for the ${ }^{209} \mathrm{Bi}(\mathrm{n}, 2 \mathrm{n}){ }^{208} \mathrm{Bi}$ reaction at $14.9 \mathrm{MeV}$ is 1.066 times the measured value at that energy, $\quad 2.20+/-0.05 \mathrm{~b}^{28}$

\section{$-24-$}

\section{References}

${ }^{1}$ D. R. Nethaway, "The Cross-Section Sets Used With the Watusi program," Internal Memo (November 5, 1998).

${ }^{2}$ H. I. West and R. G. Lanier, "Titanium Proton and Deuteron Excitation Functions," Internal memo NCD-85-58-8 (Mar. 15, 1985).

${ }^{3}$ H. West, R. Lanier, M. Mustafa, R. Nuckolls, R. Nagle, H. O’Brien, J. Frehaut, A. Adam, and C. Philis, "Some Light-Ion Excitation-Function Measurements on Titanium, Yttrium, and Europium, and Associated Results," UCRL-ID-115738 (Nov. 1993).

${ }^{4}$ S. M. Qaim and G. L. Stocklin, J. Inorg. Nucl. Chem. 35, 19 (1973).

${ }^{5}$ T. W. Woo, Trans. A.N.S. 28 , 91 (1978).

${ }^{6}$ S. M. Qaim, Nucl. Phys. A382, 255 (1982).

${ }^{7}$ H. I. West, R. G. Lanier, and M. G. Mustafa, Phys. Rev C 35 2067 (1987).

${ }^{8}$ H. I. West, R. G. Lanier, and M. G. Mustafa, "Excitation Functions Derived from Proton and Deuteron Measurements on Natural Chromium," UCID-20738 (May 1, 1986).

${ }^{9}$ H. West, R. Nuckolls, B. Hudson, B. Ruiz, R. Lanier, and M. Mustafa, Phys. Rev. C 47, 248 (1993).

${ }^{10} \mathrm{H}$. I. West, preliminary results in private communication to D. R. Nethaway (Feb. 14, 1991).

${ }^{11}$ G. Walter, et al., Nucl. Sci. Eng. 93, 357 (1986).

${ }^{12}$ F. Bazan, "(n,2n) Cross-Section Measurements of Krypton and Xenon Isotopes," Nuclear Chemistry Division Annual report, FY1988, UCAR 10062-88, p. 33 (1988).

${ }^{13}$ R. Prestwood, K. Thomas, D. Nethaway, and N. Smith, Phys. Rev. C 29, 805 (1984).

${ }^{14}$ M. Mustafa, H. West, H. O’Brien, R. Lanier, M. Benhamou, and T. Tamura, Phys. Rev. C $\underline{38}, 1624$ (1988).

${ }^{15}$ H. O'Brien, H. West, R. Lanier, and M. Mustafa, private communication (Nov. 5, 1986).

${ }^{16}$ M. Wagner, G. Winkler, H. Vonach, Cs. M. Buczko, and J. Csikai, Ann. Nucl. Eng. 16, 623 (1989).

${ }^{17}$ A. A. Delucchi and W. Goishi, "Remeasurement of ${ }^{89} \mathrm{Zr}(\mathrm{n}, 2 \mathrm{n})$ Cross Section with DT Neutrons," Memo NCD-80-18-240 (Nov. 13, 1980).

${ }^{18}$ A. Pavlik, G. Winkler, H. Vonach, A. Paulsen, and H. Liskien, J. Phys. G: Nucl. Phys. $\underline{8}$, 1283 (1982).

${ }^{19}$ R. L. Macklin, Nucl. Sci. Eng. 85, 350 (1983). 
${ }^{20}$ A. Johnsrud, M. Silbert, and H. Barschall, Phys. Rev. C_116, 927 (1959).

${ }^{21}$ H. West, R. Lanier, M. Mustafa, R. Nuckolls, J. Frehaut, A. Adam, and C. Philis, "Preliminary Results for the Excitation Functions of ${ }^{151,153} \mathrm{Eu}(\mathrm{p}, \mathrm{n}){ }^{151,153} \mathrm{Gd}$ and ${ }^{151,153} \mathrm{Eu}(\mathrm{d}, 2 \mathrm{n}){ }^{151,153} \mathrm{Gd}$ : Measurements and Modeling," Nuclear Chemistry Division Annual Report, FY1988, UCAR 10062-88, P. 37 (1988).

${ }^{22}$ H. I. West, private communication to D. R. Nethaway (July 28, 1988).

${ }^{23}$ R. L. Macklin and P. G. Young, Nucl. Sci. Eng. 95, 189 (1987).

${ }^{24}$ P. G. Young and E. D. Arthur, Preliminary Evaluation for ENDF/B-6, private communication from LANL to D. R. Nethaway (Apr. 21, 1986).

${ }^{25}$ S. M. Qaim, Nucl. Phys. A224, 319 (1974).

${ }^{26}$ H. Beer and R. L. Macklin, Astrophysical Jour. 331, 1047 (1988).

${ }^{27}$ D. R. Nethaway, "Cross-Section Measurements for Radioactive targets," Nuclear Chemistry Division Annual Report, FY 1980, UCAR-10062, Pt. 1, p. IV-A-10 (1980).

${ }^{28}$ A. J. Gancarz, G. W. Butler, and R. J. Prestwood, "Bismuth Cross Sections," LANL Memo CNC7-7/81-143, (July 10, 1981). 


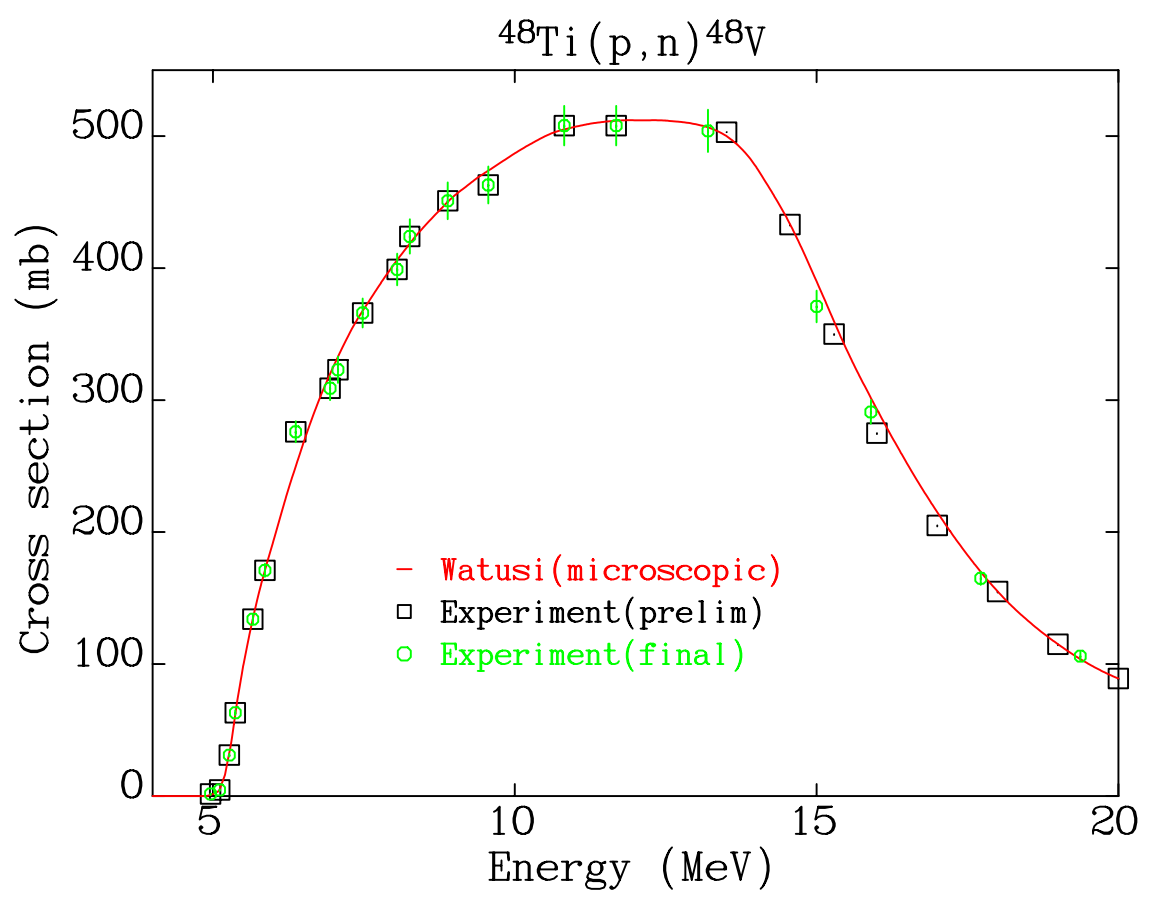

Fig. $1 .{ }^{48} \mathrm{Ti}(\mathrm{p}, \mathrm{n})^{48} \mathrm{~V}$. The Watusi (microscopic) cross sections (red line) obtained from a fit to the preliminary experimental data (Table 2) are shown against both the preliminary (black squares) and the final (green circles) experimental data (Table 5). Note that the two sets of data are identical below $12 \mathrm{MeV}$ and have negligible difference above that energy.

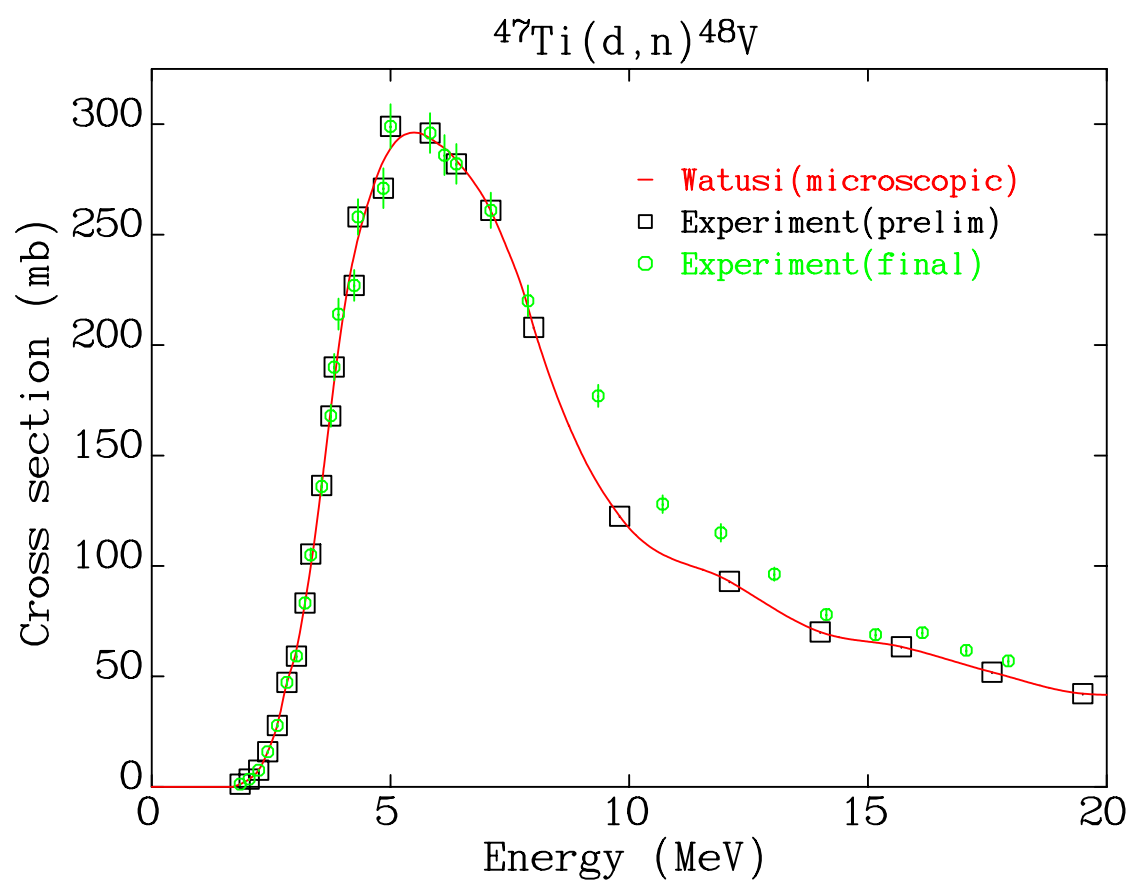

Fig. 2. ${ }^{47} \mathrm{Ti}(\mathrm{d}, \mathrm{n})^{48} \mathrm{~V}$. The Watusi (microscopic) cross sections (red line) obtained from a fit to the preliminary experimental data (Table 3 ) are shown against both the preliminary (black squares) and the final (green circles) experimental data (Table 6). Note that the two sets of data are identical below $7 \mathrm{MeV}$. The Watusi cross sections above $8 \mathrm{MeV}$ will need revision. 


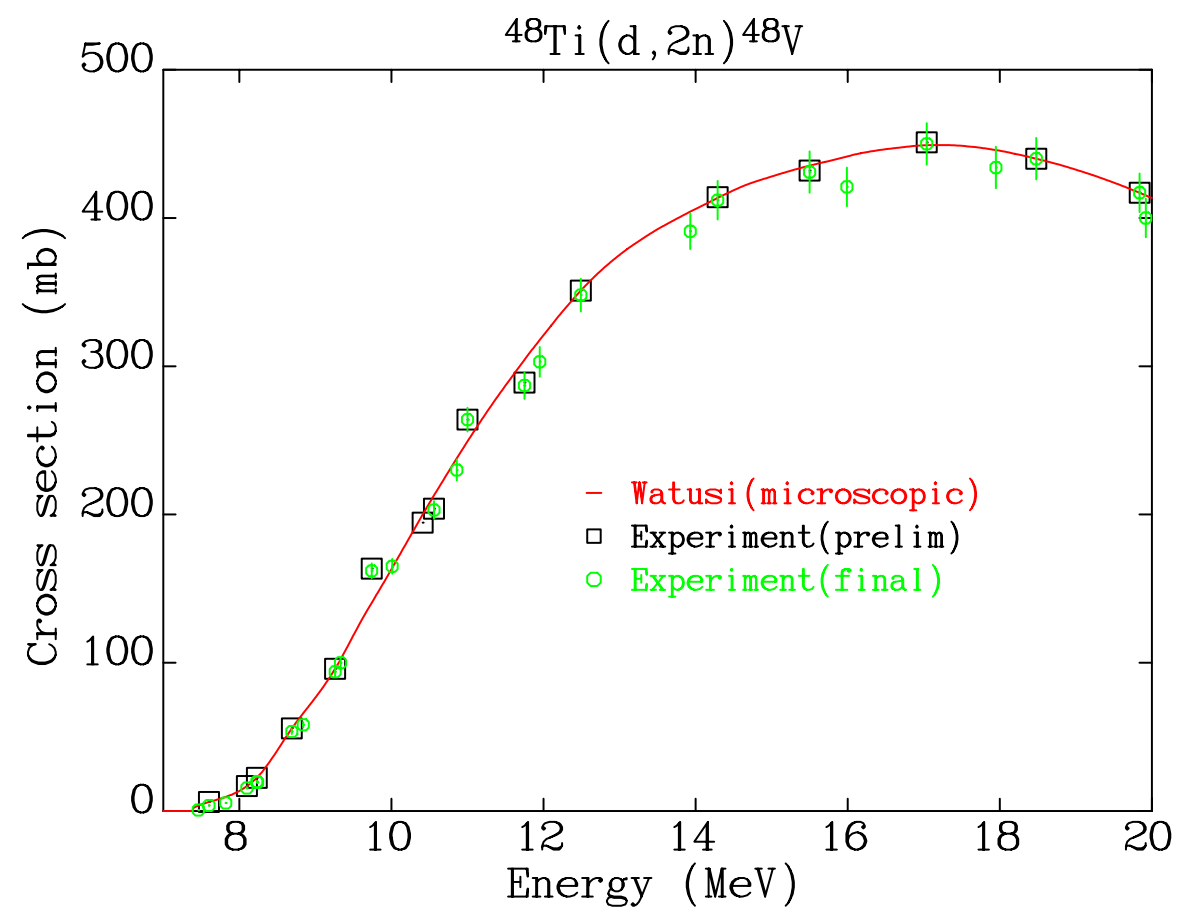

Fig. 3. ${ }^{48} \mathrm{Ti}(\mathrm{d}, 2 \mathrm{n})^{48} \mathrm{~V}$. The Watusi (microscopic) cross sections (red line) obtained from a fit to the preliminary experimental data (Table 4) are shown against both the preliminary (black squares) and the final (green circles) experimental data (Table 7). Note that the Watusi cross sections above $12 \mathrm{MeV}$ will need revision.

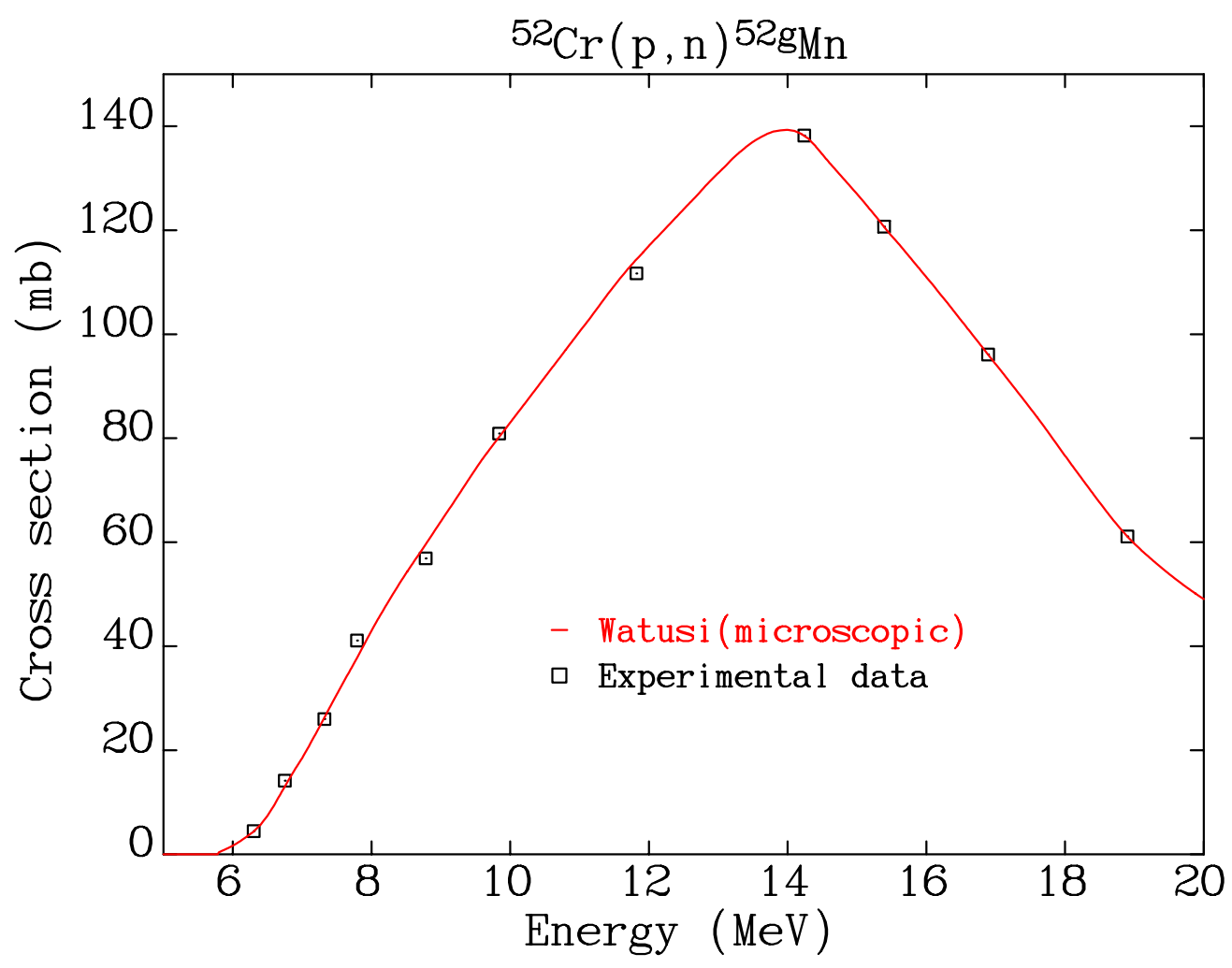

Fig. 4. ${ }^{52} \mathrm{Cr}(\mathrm{p}, \mathrm{n}){ }^{52 \mathrm{~g}} \mathrm{Mn}$. The Watusi (microscopic) cross sections (red line) are plotted against the experimental data (Table 12). 


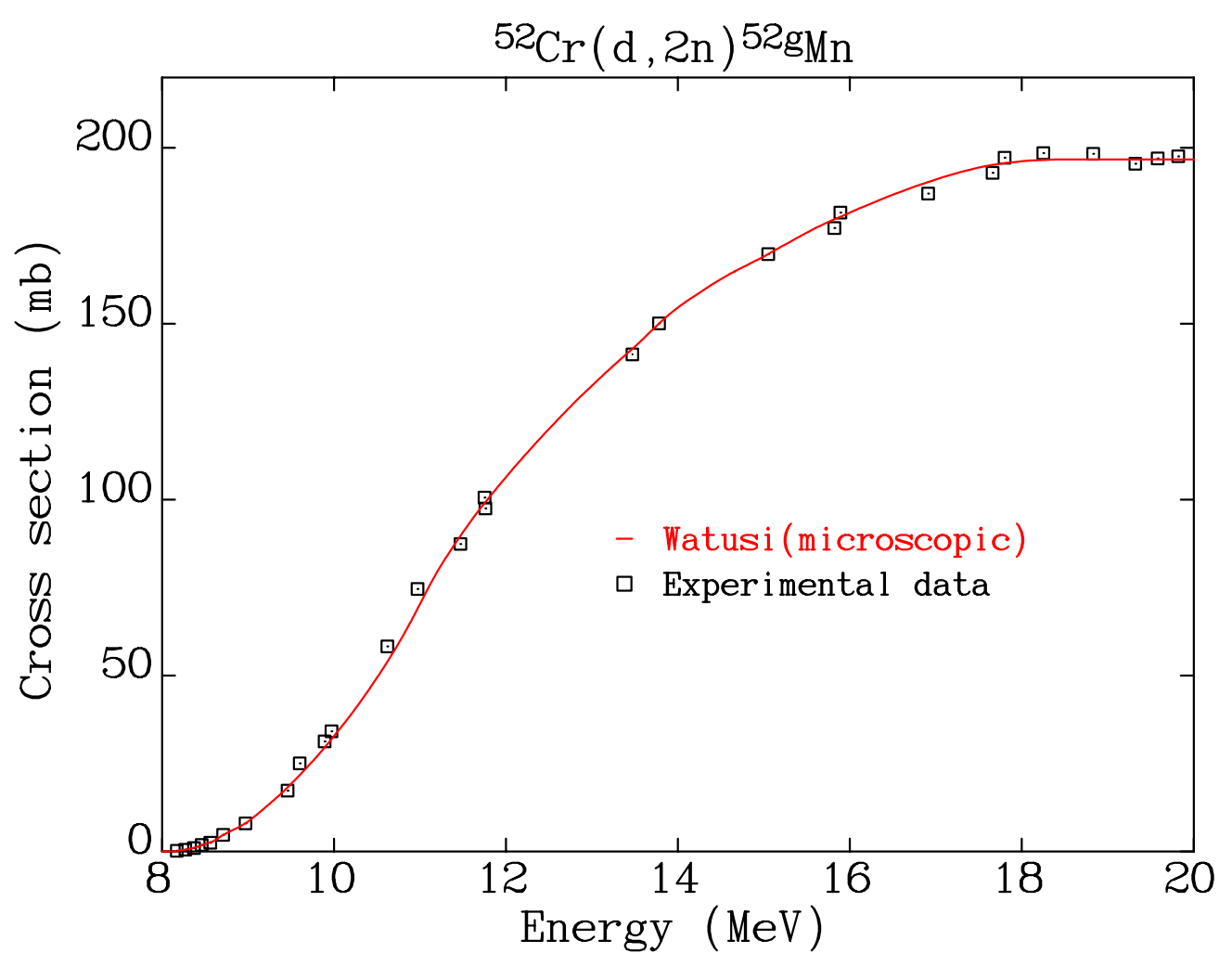

Fig. 5. ${ }^{52} \mathrm{Cr}(\mathrm{d}, 2 \mathrm{n}){ }^{52 \mathrm{~g}} \mathrm{Mn}$. The Watusi (microscopic) cross sections (red line) are plotted against the experimental data (Table 13).

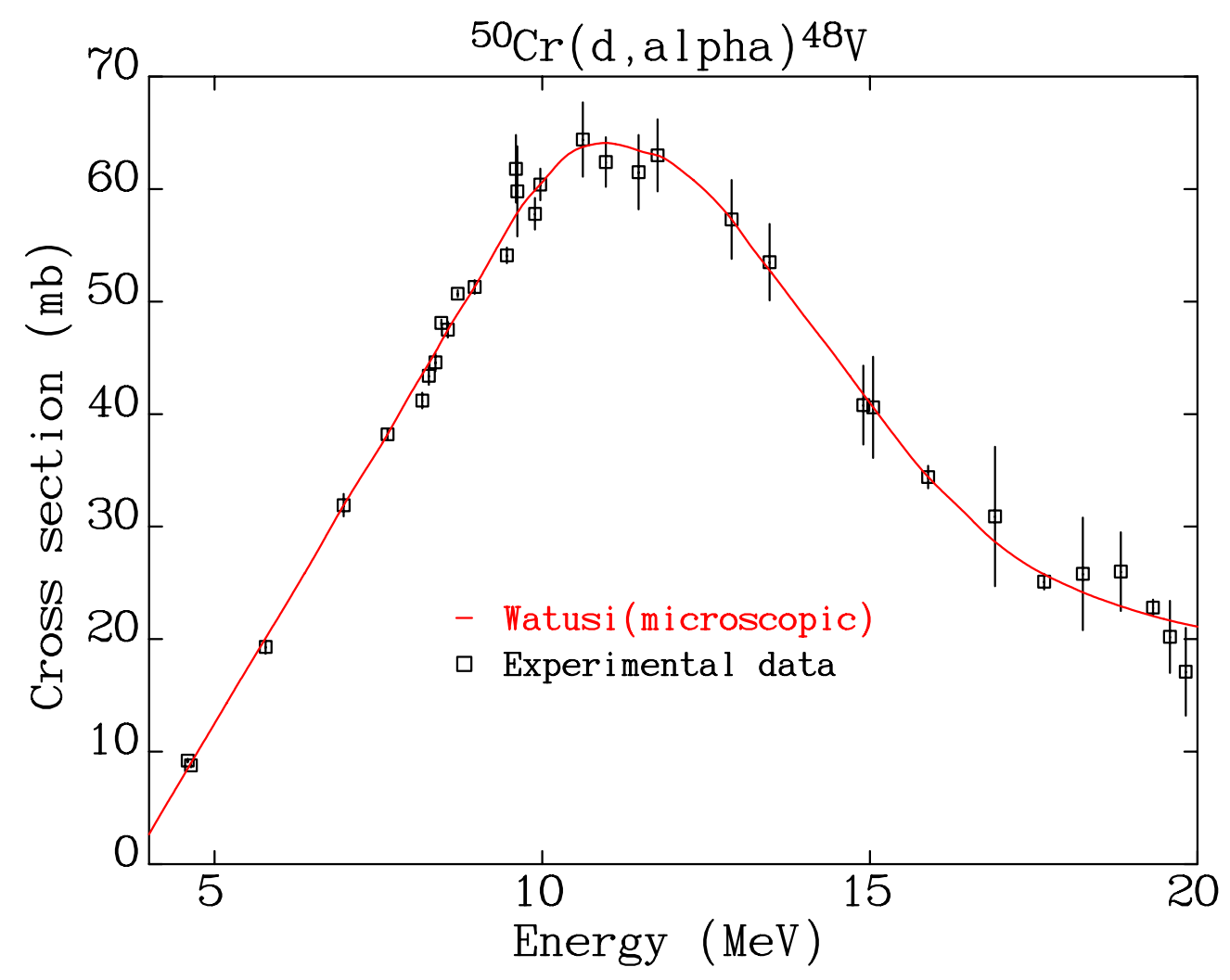

Fig. 6. ${ }^{50} \mathrm{Cr}(\mathrm{d} \text {,alpha })^{48} \mathrm{~V}$. The Watusi (microscopic) cross sections (red line) are plotted against the experimental data (Table 14). 


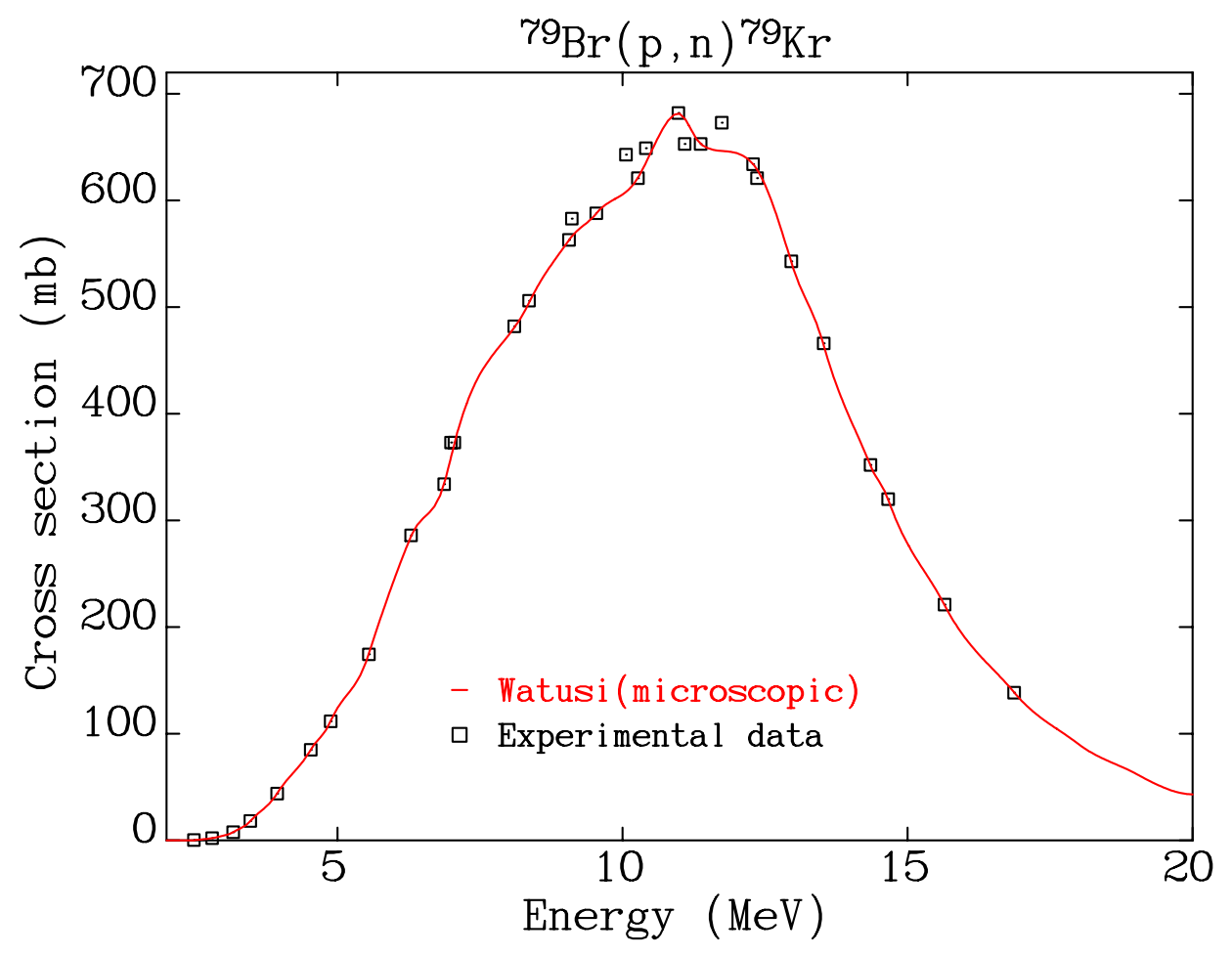

Fig. 7. ${ }^{79} \mathrm{Br}(\mathrm{p}, \mathrm{n}){ }^{79} \mathrm{Kr}$. The Watusi (microscopic) cross sections (red line) are plotted against experimental data (Table 15).

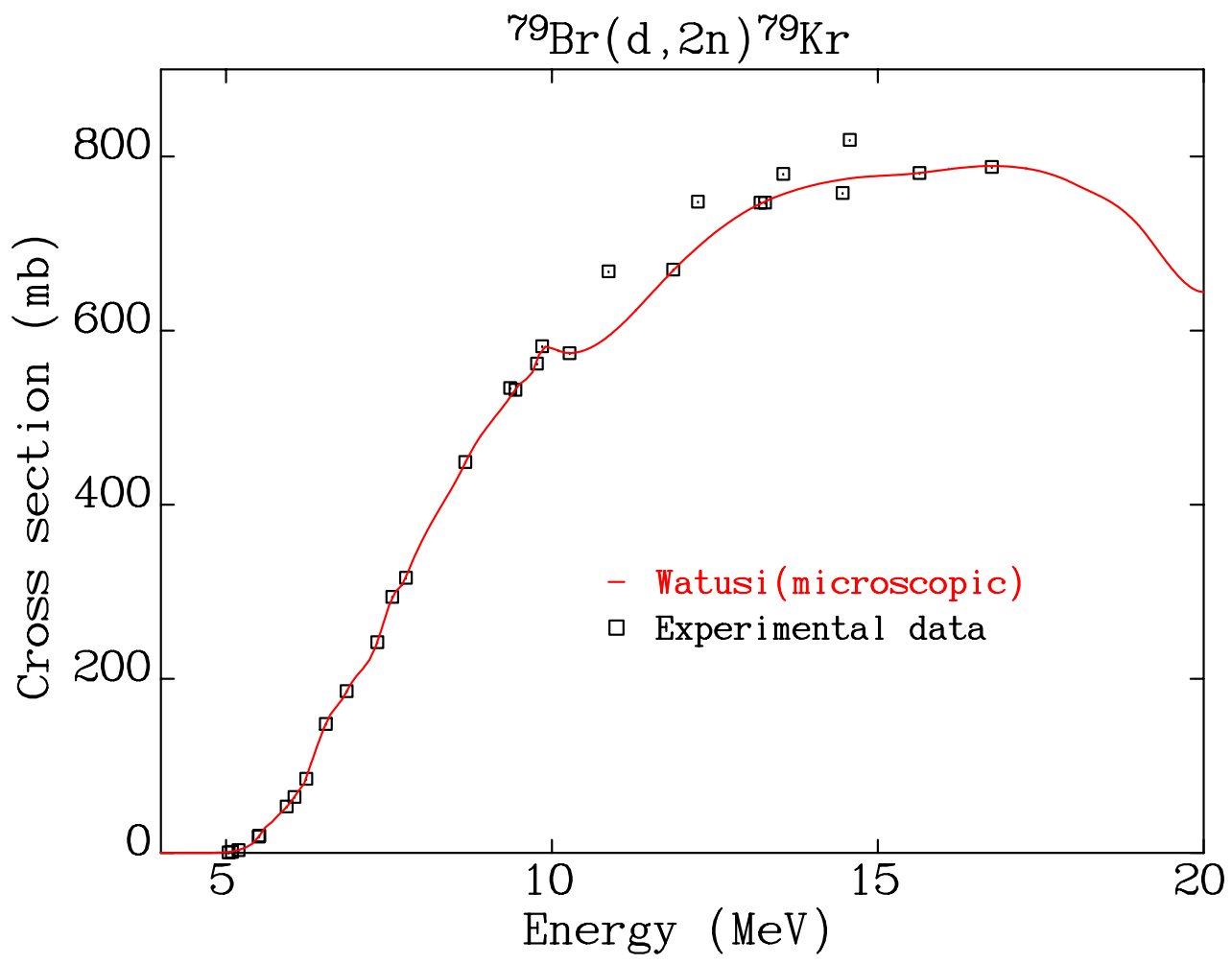

Fig. 8. ${ }^{79} \mathrm{Br}(\mathrm{d}, 2 \mathrm{n}){ }^{79} \mathrm{Kr}$. The Watusi (microscopic) cross sections (red line) are plotted against the experimental data (Table 16). 


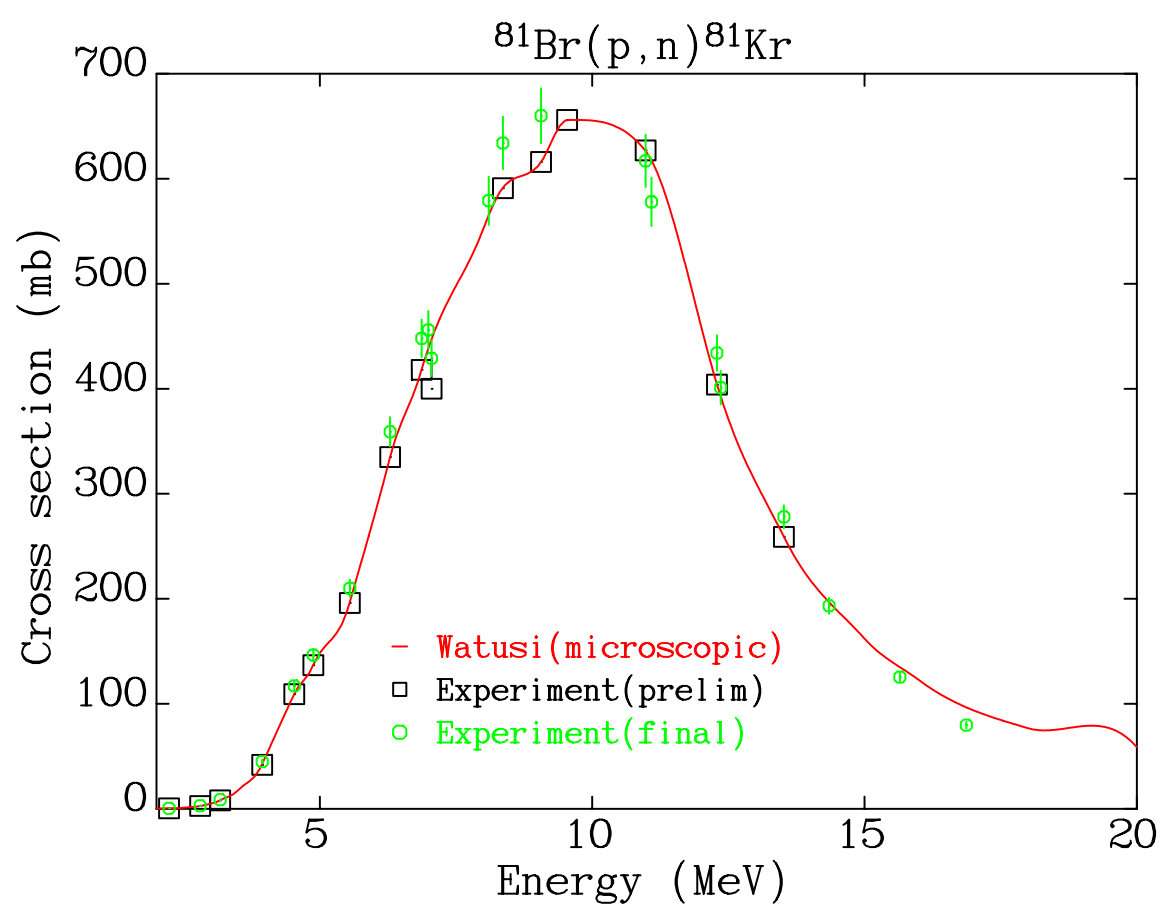

Fig. 9. ${ }^{81} \mathrm{Br}(\mathrm{p}, \mathrm{n}){ }^{81} \mathrm{Kr}$. The Watusi (microscopic) cross sections (red line) obtained from a fit to the preliminary experimental data are shown against both the preliminary (black squares) and final (green circles) experimental data (both sets of data are in Table 17).

Note that the Watusi cross sections will need revision.

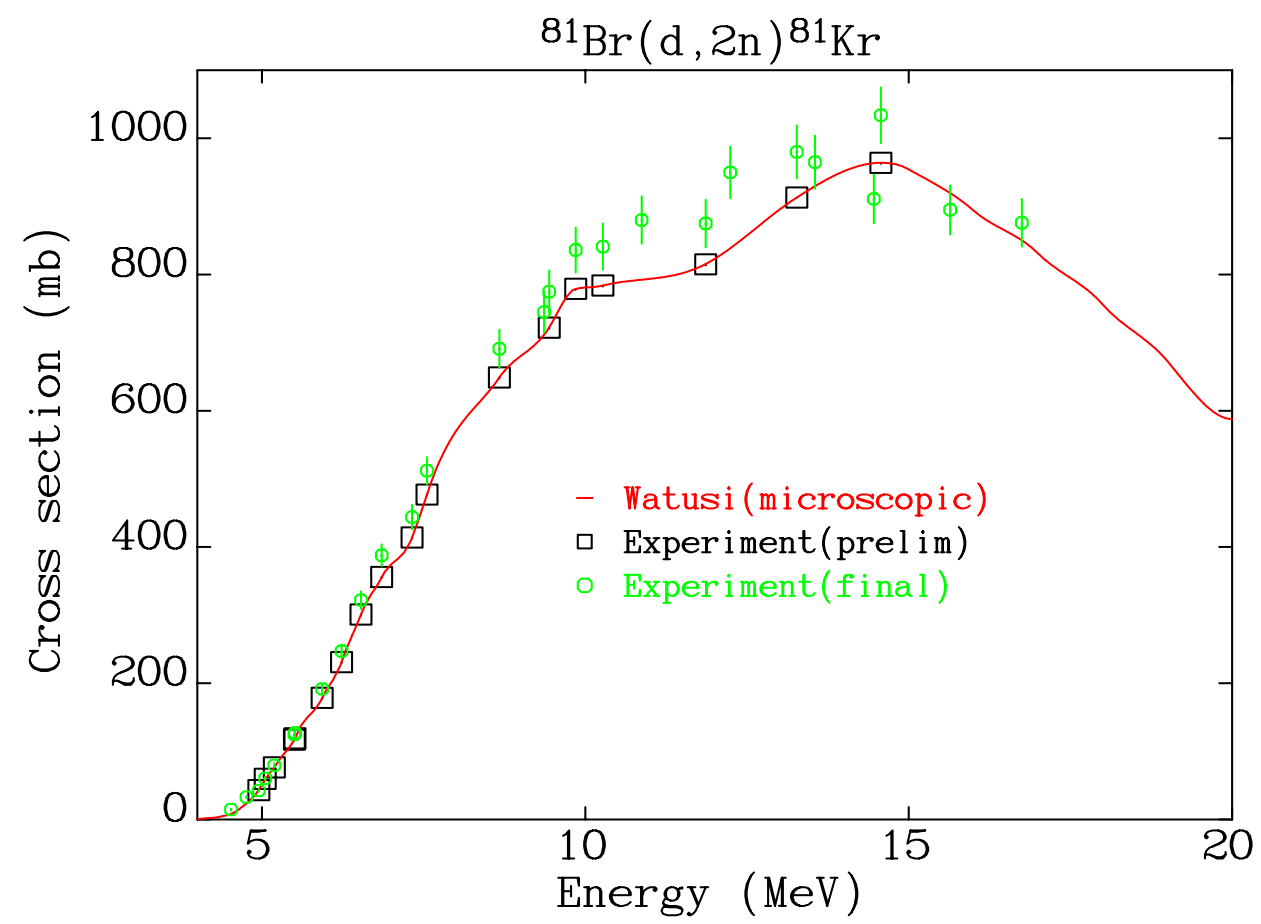

Fig. 10. ${ }^{81} \mathrm{Br}(\mathrm{d}, 2 \mathrm{n}){ }^{81} \mathrm{Kr}$. The Watusi (microscopic) cross sections (red line) obtained from a fit to the preliminary experimental data are shown against both the preliminary (black squares) and final (green circles) experimental data (both sets of data are in Table 18).

Note that the Watusi cross sections will need revision. 


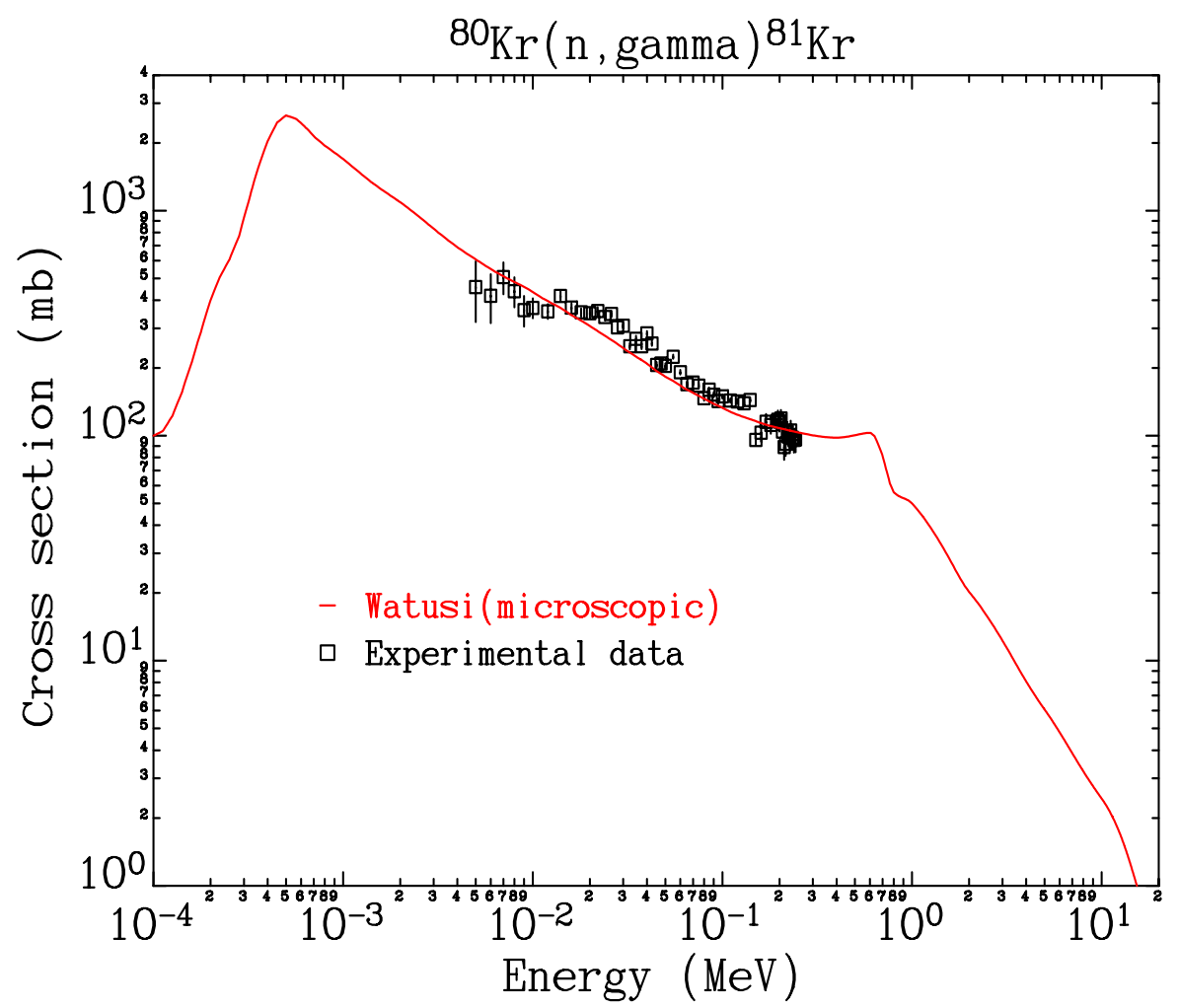

Fig. 11. ${ }^{80} \mathrm{Kr}(\mathrm{n}, \mathrm{g}){ }^{81} \mathrm{Kr}$. The Watusi (microscopic) cross sections (red line) are plotted against the experimental data (Table 19).

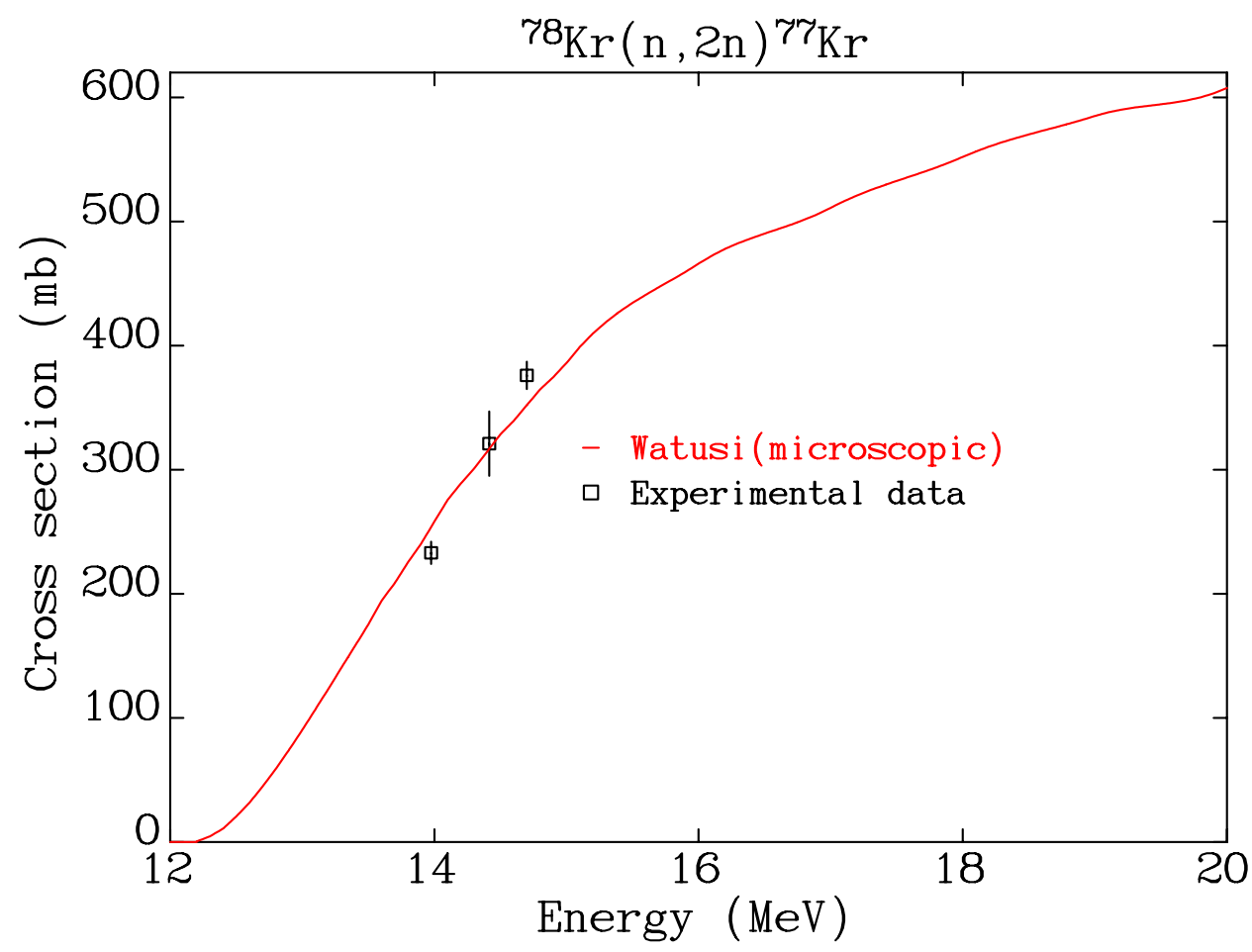

Fig. 12. ${ }^{78} \mathrm{Kr}(\mathrm{n}, 2 \mathrm{n}){ }^{77} \mathrm{Kr}$. The Watusi (microscopic) cross sections (red line) are plotted against the experimental data (Table 20). 


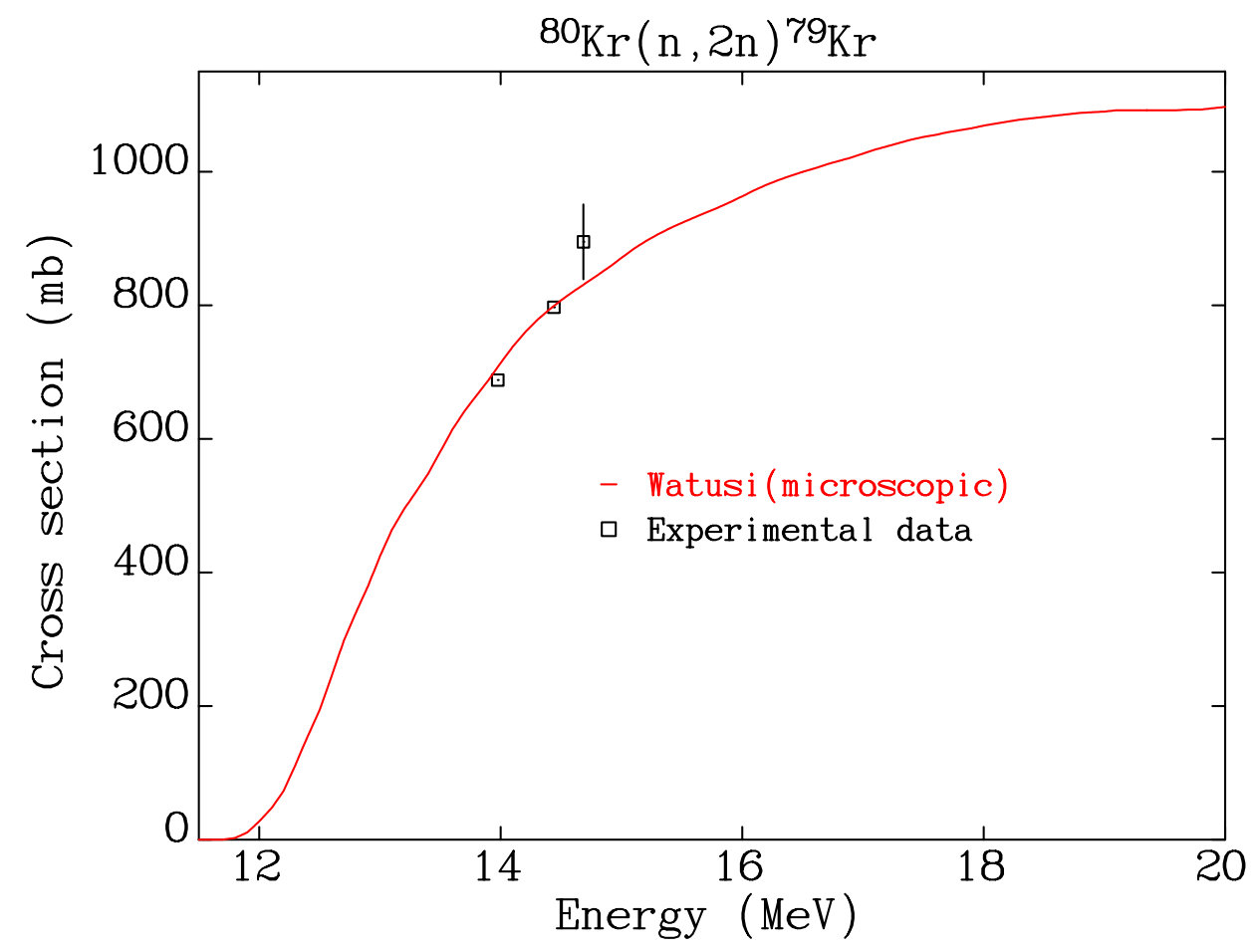

Fig. 13. ${ }^{80} \mathrm{Kr}(\mathrm{n}, 2 \mathrm{n}){ }^{79} \mathrm{Kr}$. The Watusi (microscopic) cross sections (red line) are plotted against the experimental data (Table 20).

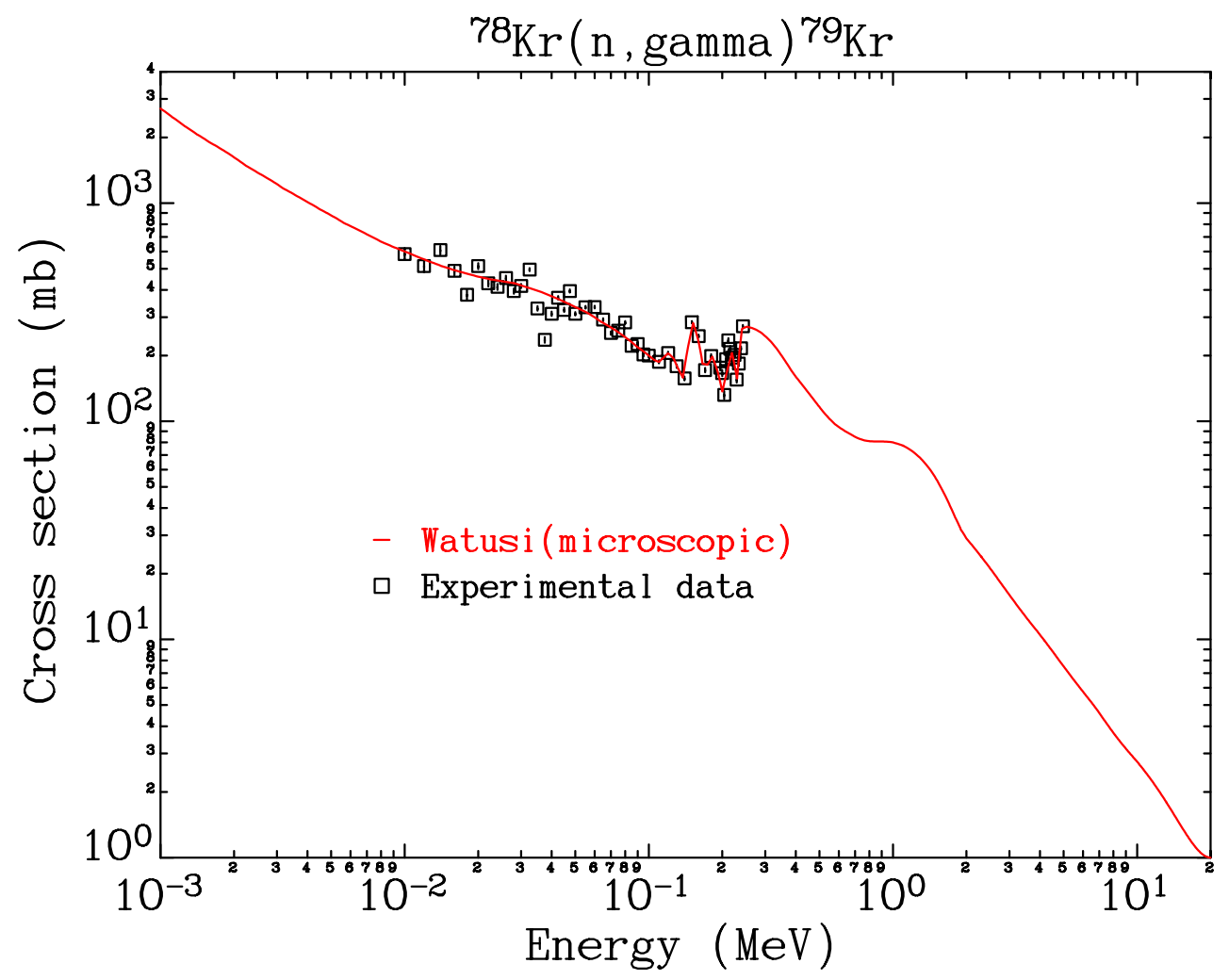

Fig. 14. ${ }^{78} \mathrm{Kr}(\mathrm{n}, \mathrm{g}){ }^{79} \mathrm{Kr}$. The Watusi (microscopic) cross sections (red line) are plotted against the experimental data (Table 21). 


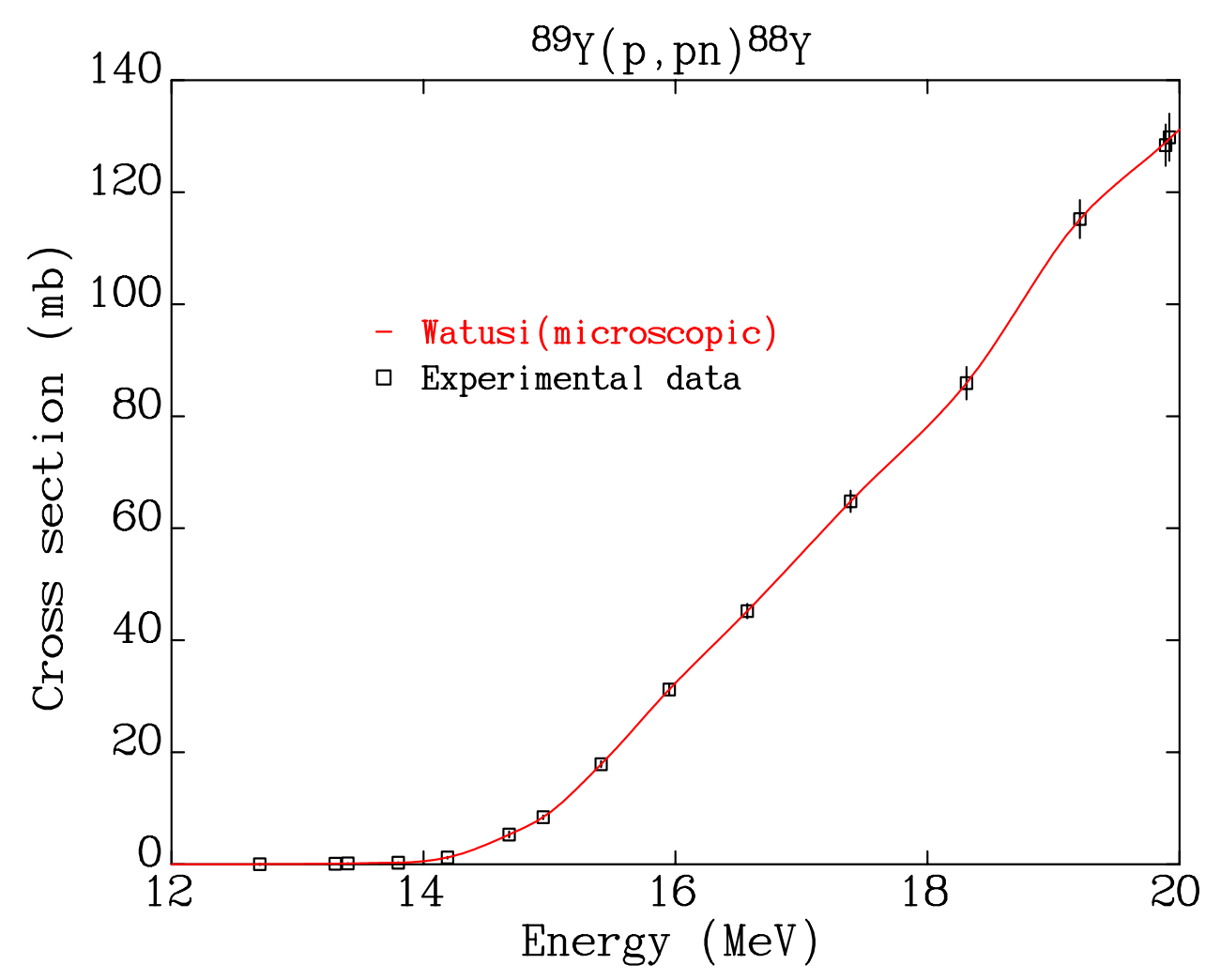

Fig. 15. ${ }^{89} \mathrm{Y}(\mathrm{p}, \mathrm{pn})^{88} \mathrm{Y}$. The Watusi (microscopic) cross sections (red line) are plotted against the experimental data (Table 22).

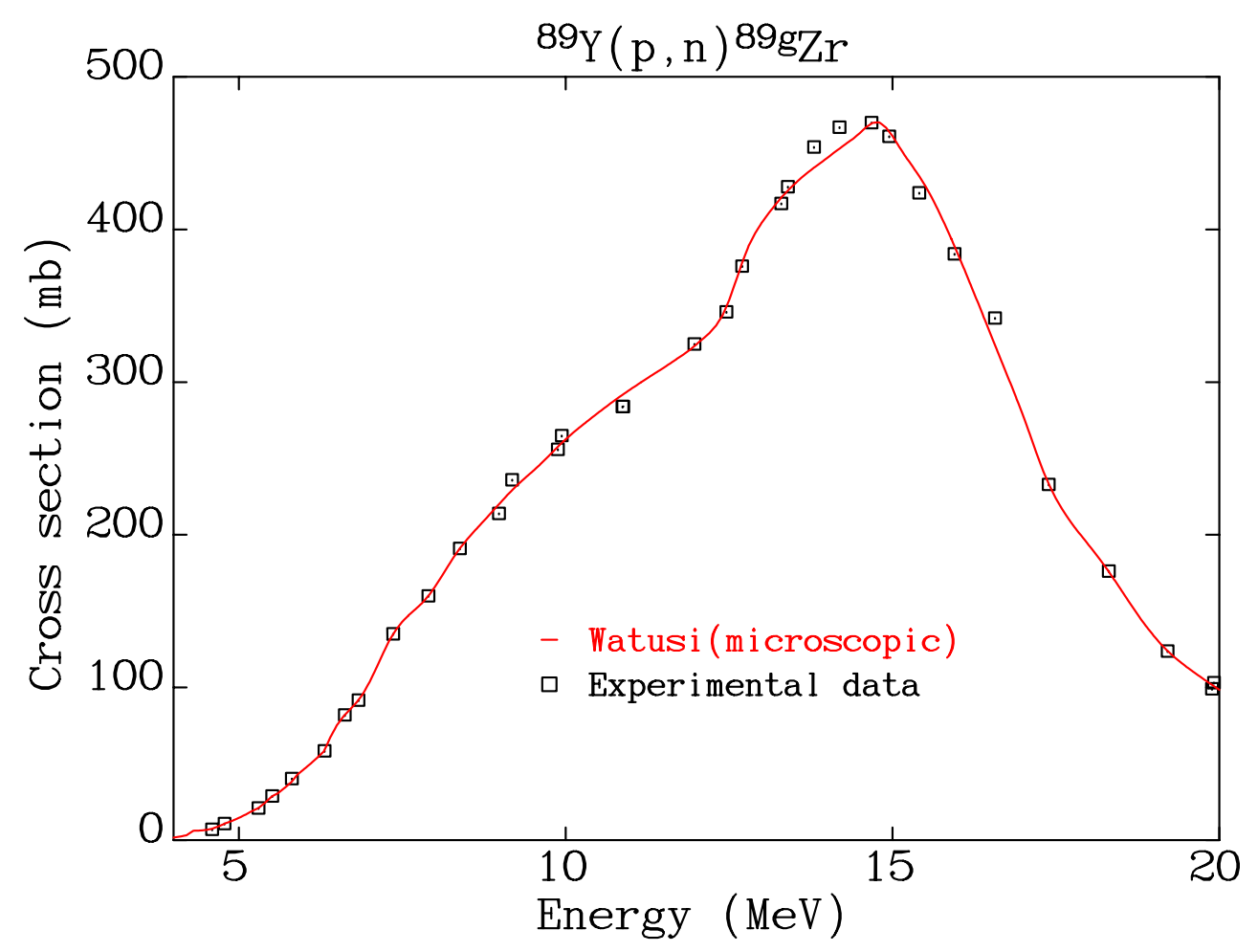

Fig. 16. ${ }^{89} \mathrm{Y}(\mathrm{p}, \mathrm{n})^{89 \mathrm{~g}} \mathrm{Zr}$. The Watusi (microscopic) cross sections (red line) are plotted against the experimental data (Table 23). 


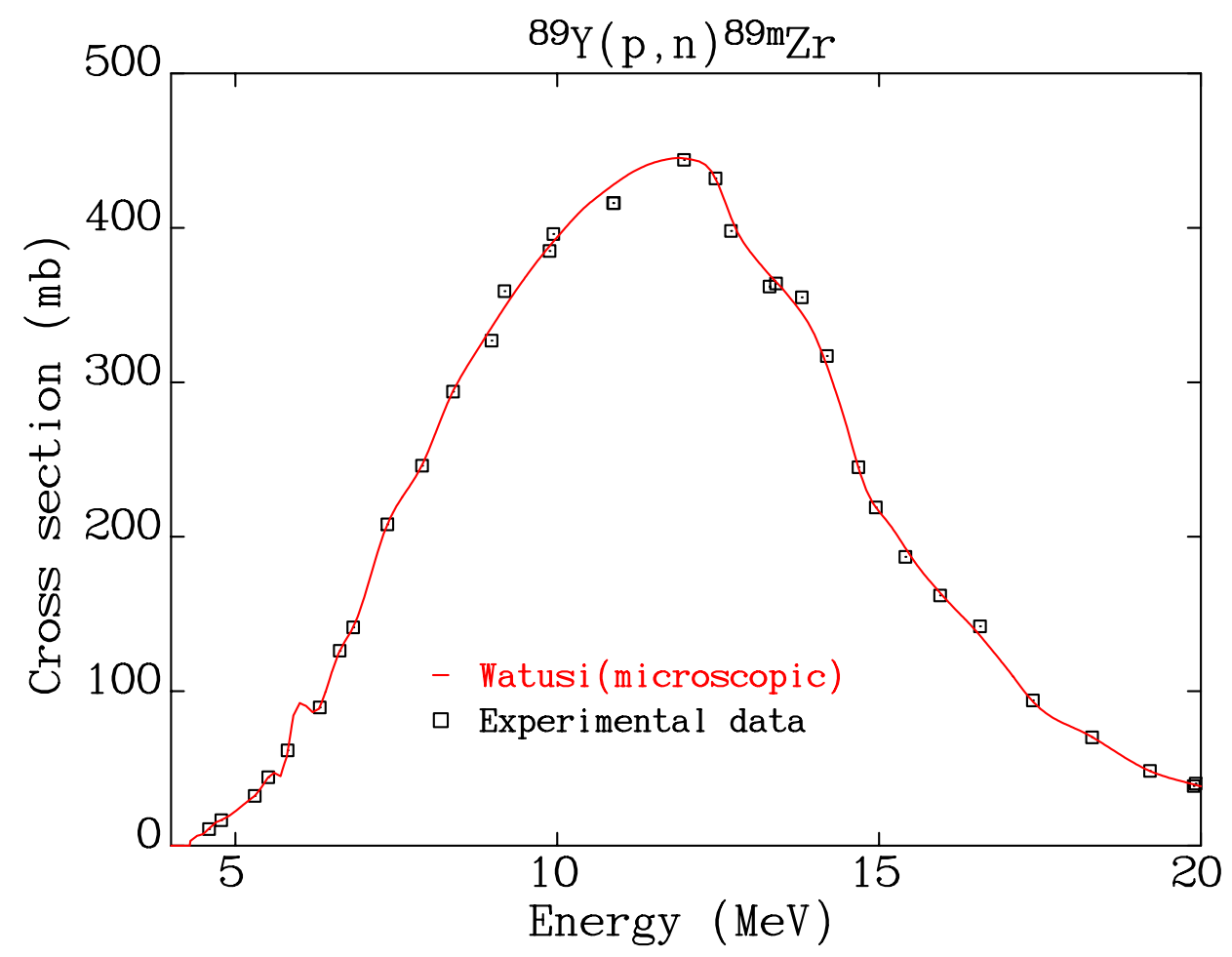

Fig. 17. ${ }^{89} \mathrm{Y}(\mathrm{p}, \mathrm{n}){ }^{89 \mathrm{~m}} \mathrm{Zr}$. The Watusi (microscopic) cross sections (red line) are plotted against the experimental data (Table 23).

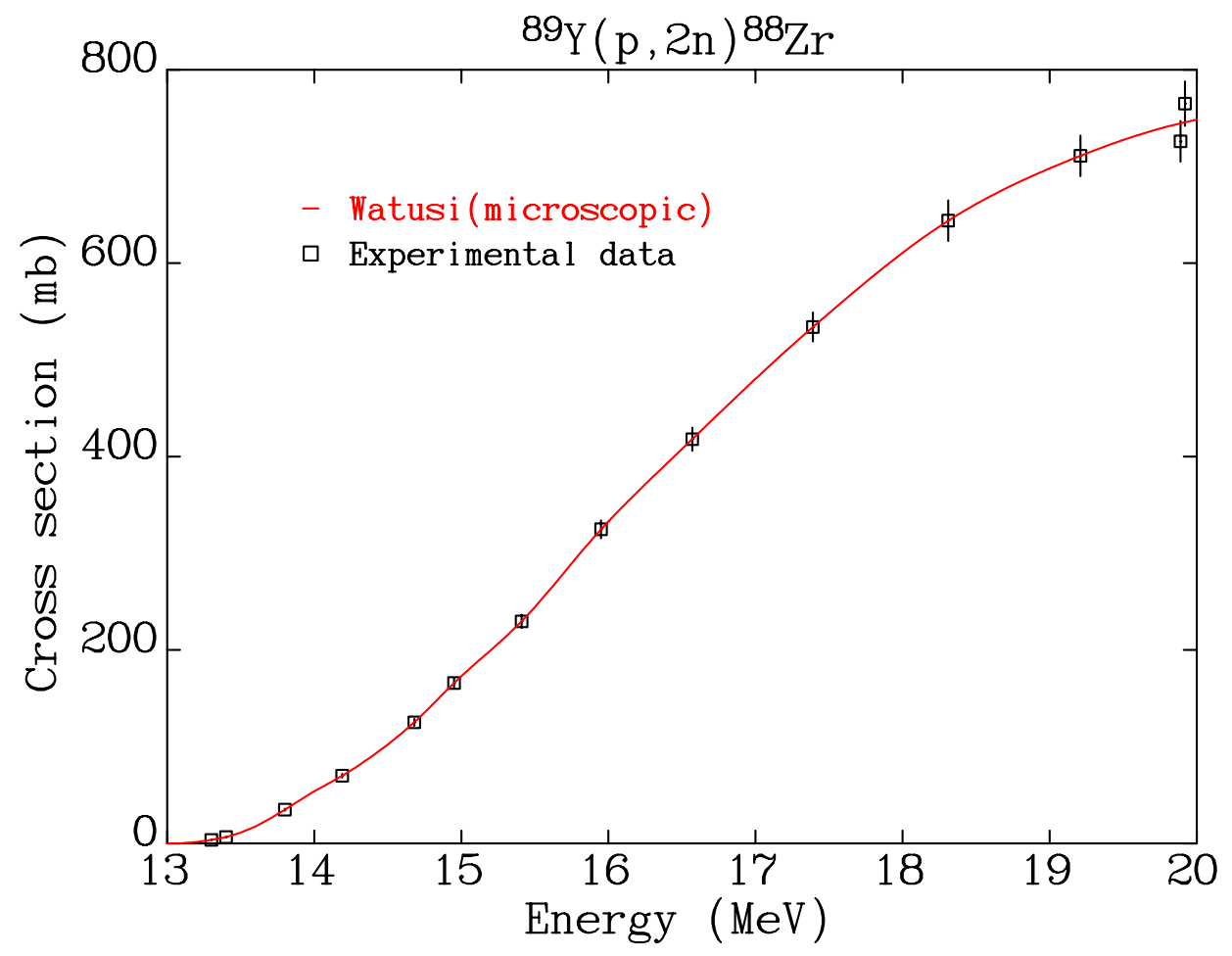

Fig. 18. ${ }^{89} \mathrm{Y}(\mathrm{p}, 2 \mathrm{n}){ }^{88} \mathrm{Zr}$. The Watusi (microscopic) cross sections (red line) are plotted against the experimental data (Table 24). 


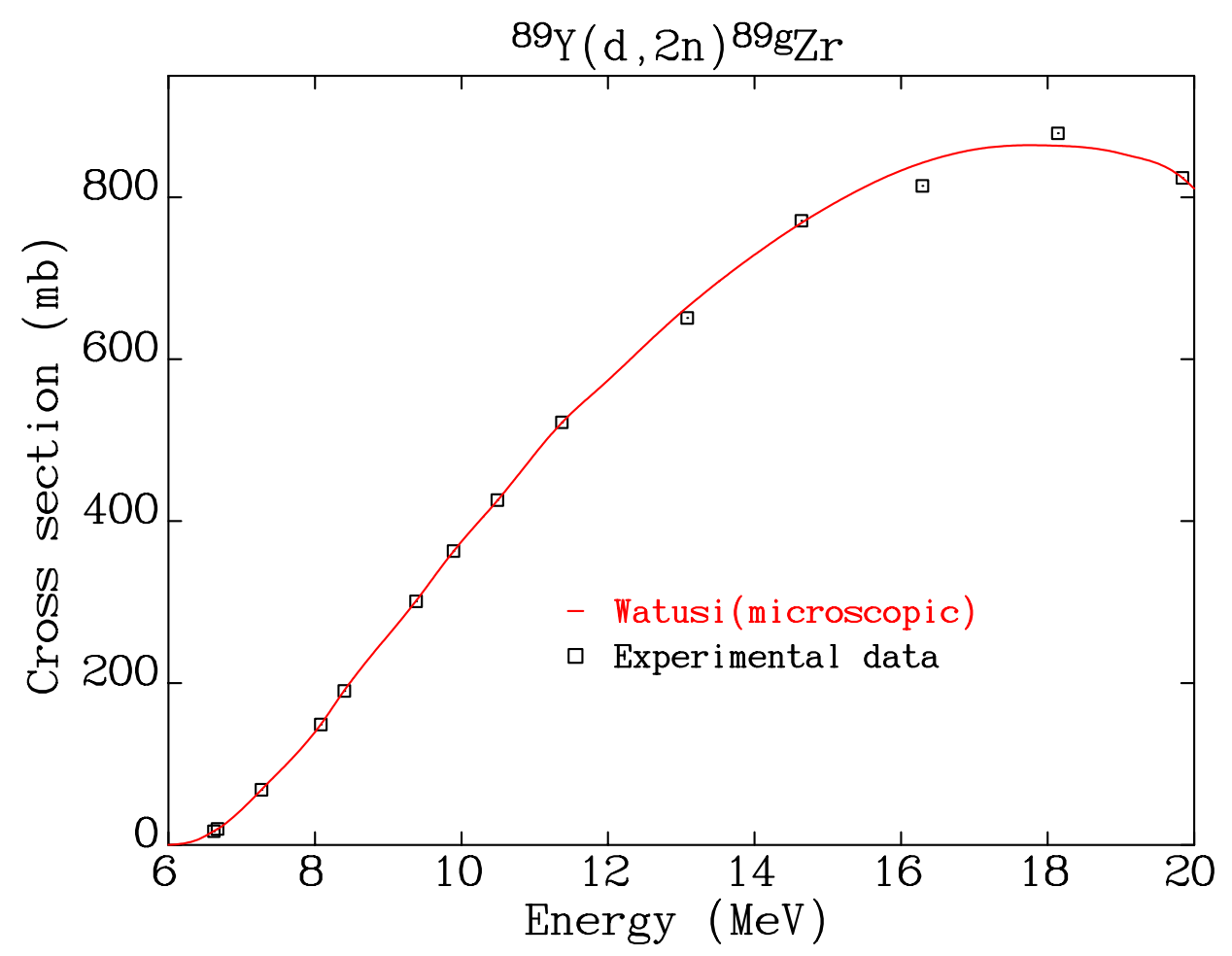

Fig. 19. ${ }^{89} \mathrm{Y}(\mathrm{d}, 2 \mathrm{n}){ }^{89 \mathrm{~g}} \mathrm{Zr}$. The Watusi (microscopic) cross sections (red line) are plotted against the experimental data (Table 25).

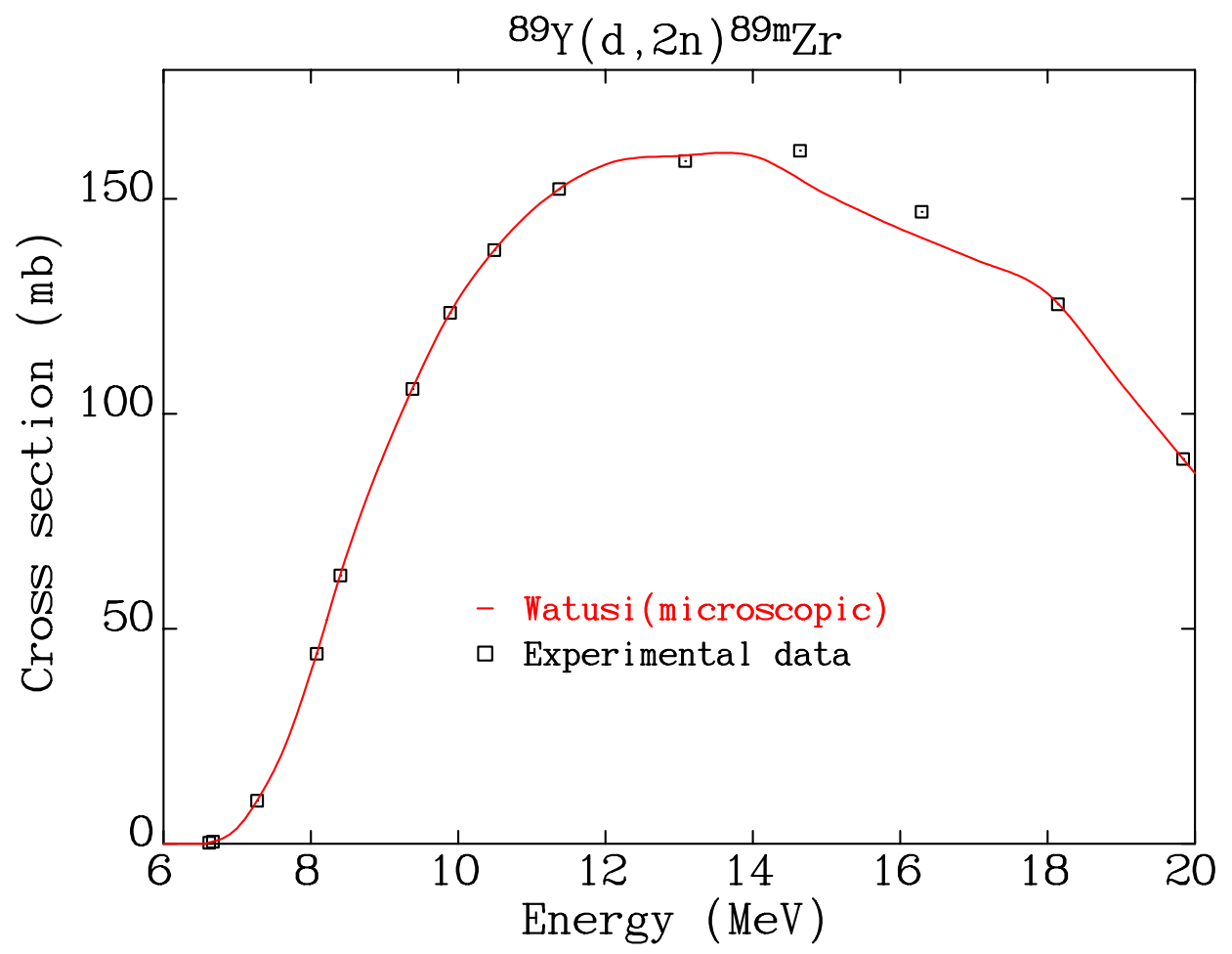

Fig. 20. ${ }^{89} \mathrm{Y}(\mathrm{d}, 2 \mathrm{n})^{89 \mathrm{~m}} \mathrm{Zr}$. The Watusi (microscopic) cross sections (red line) are plotted against the experimental data (Table 25). 


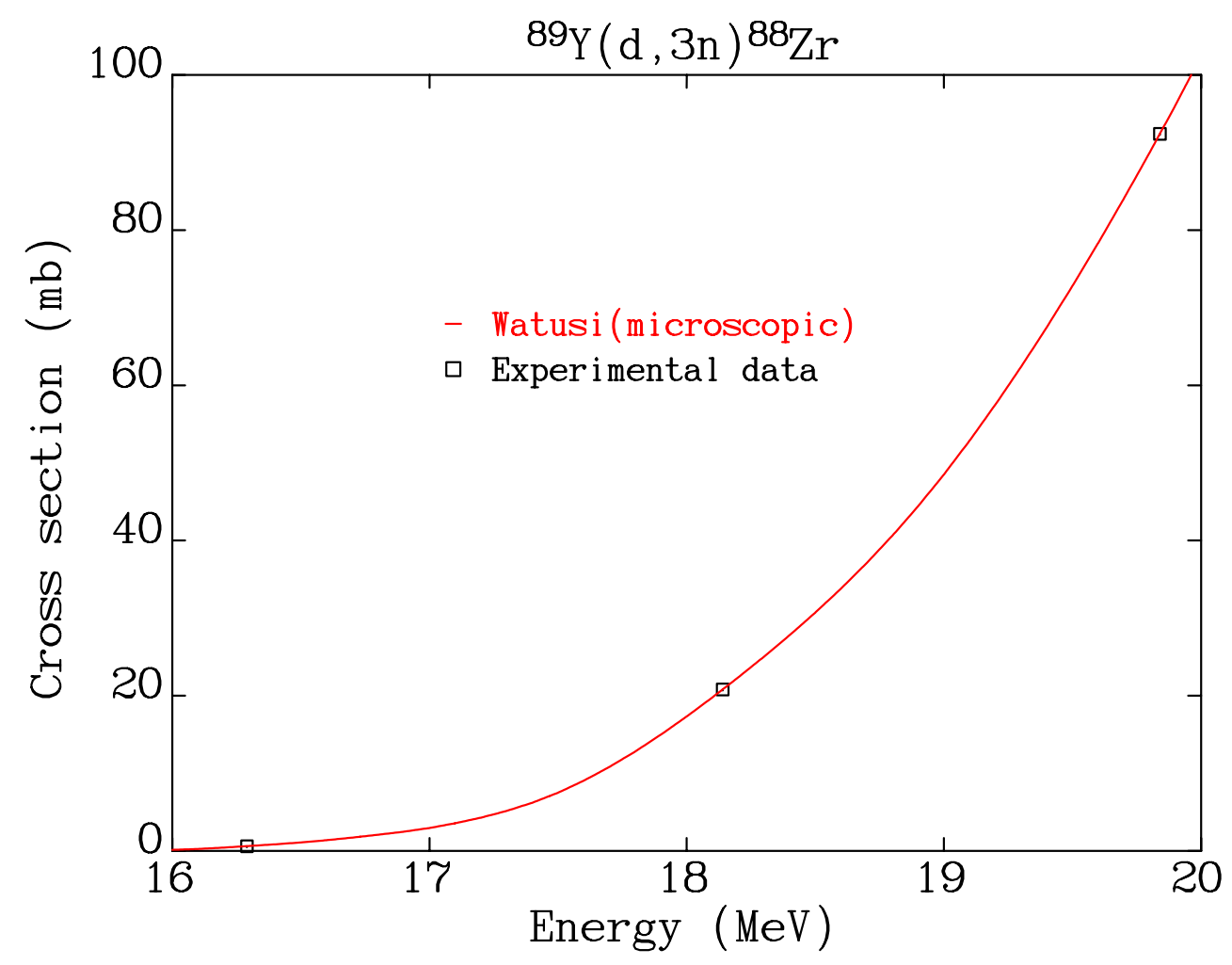

Fig. 21. ${ }^{89} \mathrm{Y}(\mathrm{d}, 3 \mathrm{n}){ }^{88} \mathrm{Zr}$. The Watusi (microscopic) cross sections (red line) are plotted against the experimental data (Table 26).

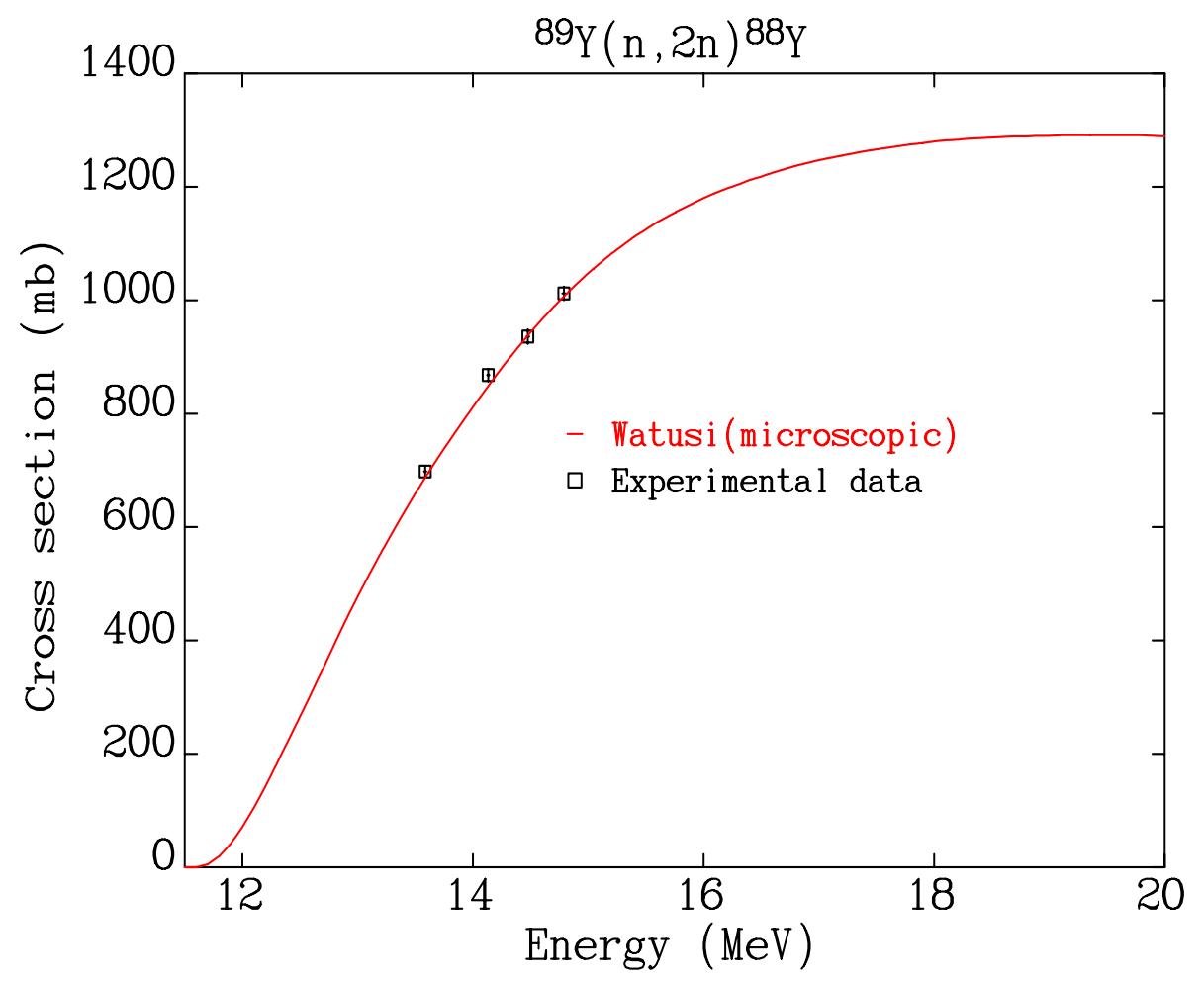

Fig. 22. ${ }^{89} \mathrm{Y}(\mathrm{n}, 2 \mathrm{n}){ }^{88} \mathrm{Y}$. The Watusi (microscopic) cross sections (red line) are plotted against the experimental data (Table 29). 


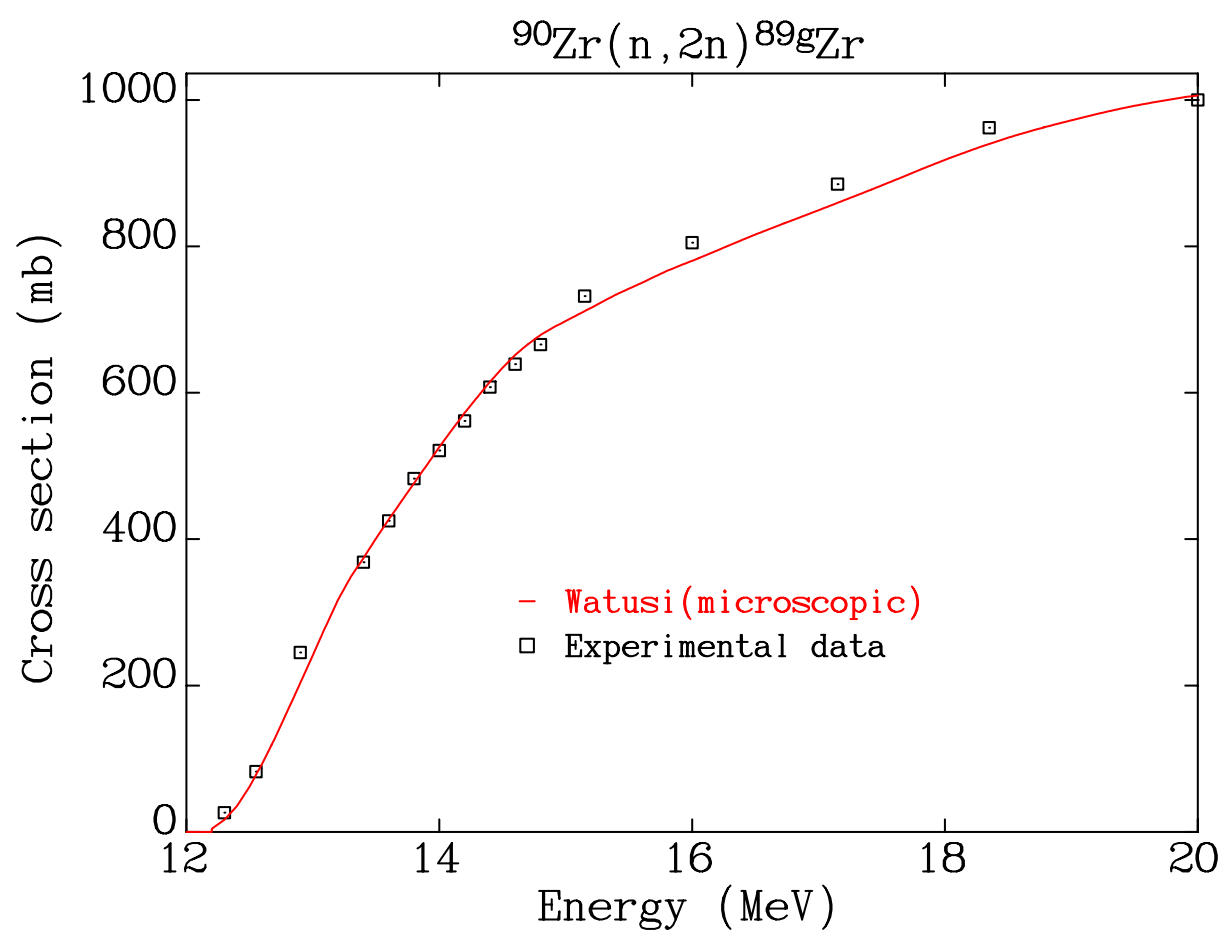

Fig. 23. ${ }^{90} \mathrm{Zr}(\mathrm{n}, 2 \mathrm{n})^{89 \mathrm{~g}} \mathrm{Zr}$. The Watusi (microscopic) cross sections (red line) are plotted against the experimental data (Table 30).

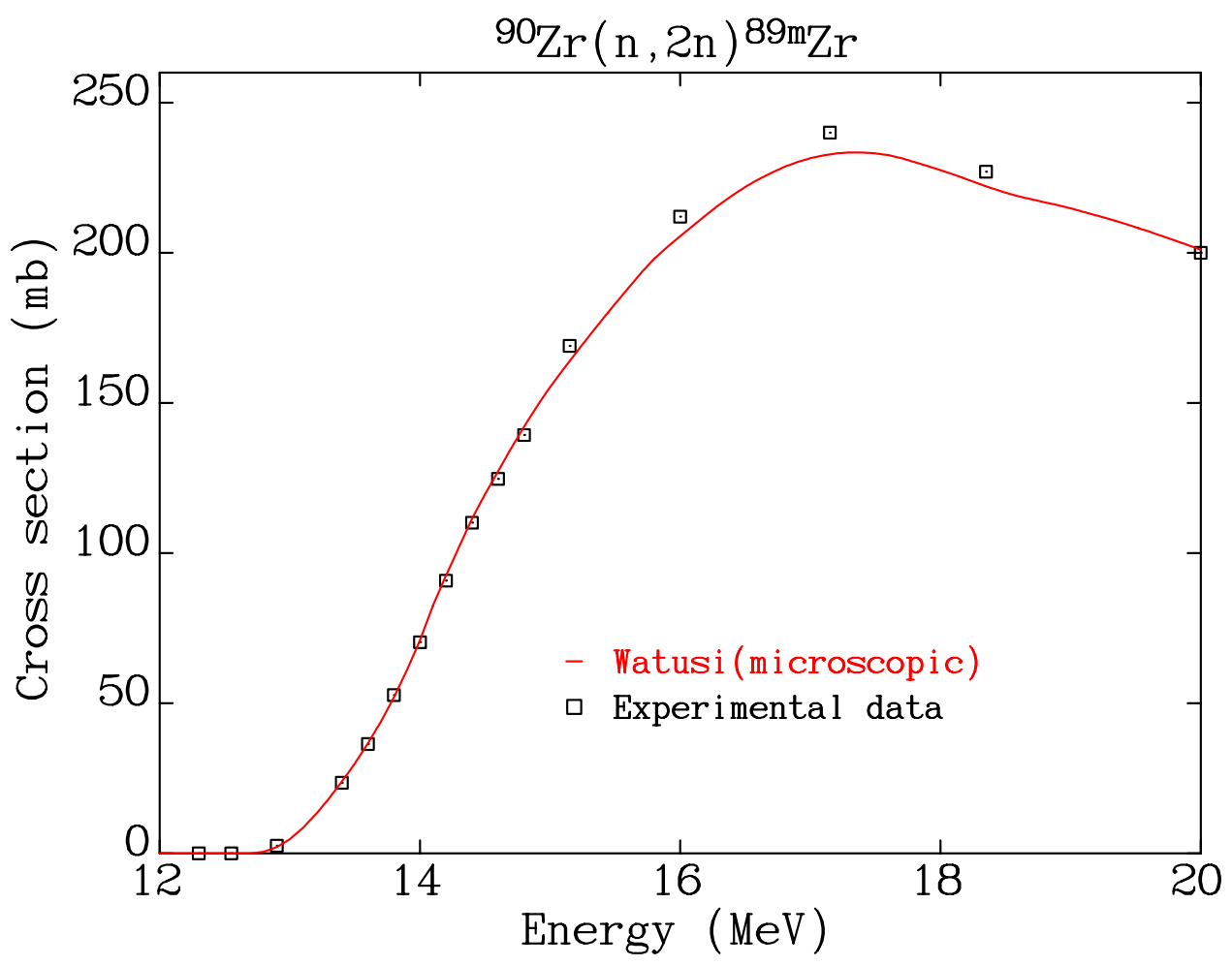

Fig. 24. ${ }^{90} \mathrm{Zr}(\mathrm{n}, 2 \mathrm{n})^{89 \mathrm{~m}} \mathrm{Zr}$. The Watusi (microscopic) cross sections (red line) are plotted against the experimental data (Table 30). 


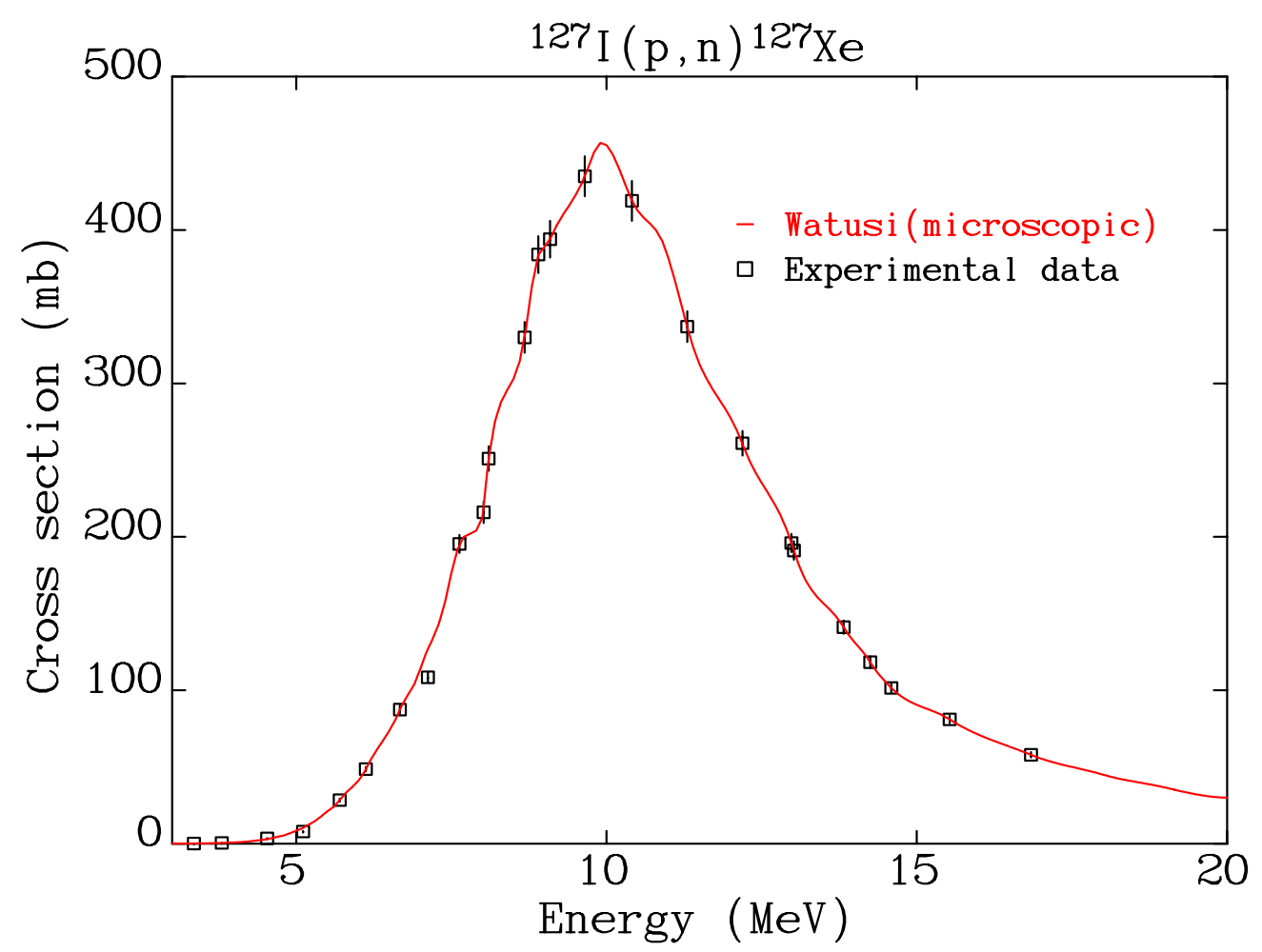

Fig. 25. ${ }^{127} \mathrm{I}(\mathrm{p}, \mathrm{n}){ }^{127} \mathrm{Xe}$. The Watusi (microscopic) cross sections (red line) are plotted against the experimental data (Table 31).

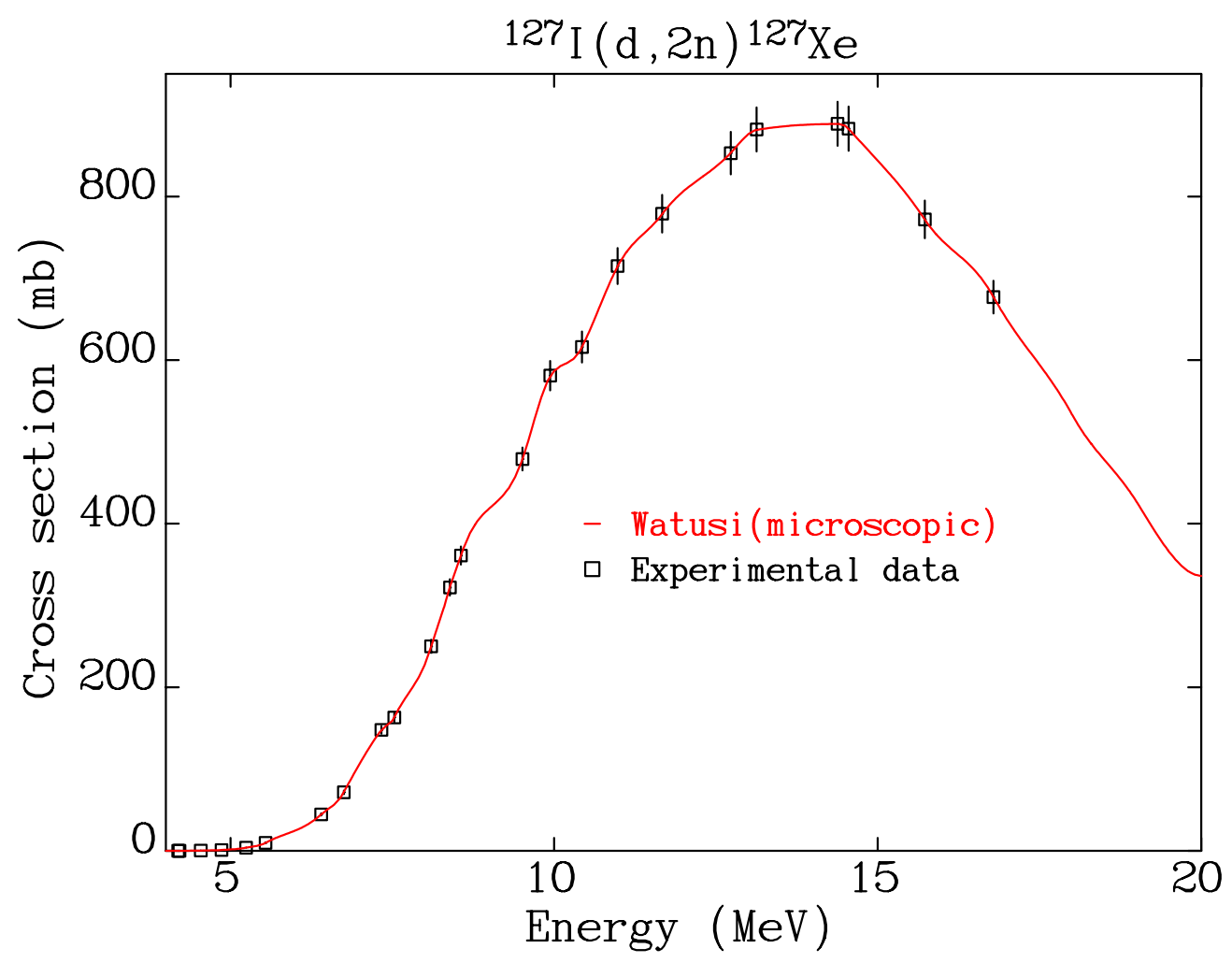

Fig. 26. ${ }^{127} \mathrm{I}(\mathrm{d}, 2 \mathrm{n}){ }^{127} \mathrm{Xe}$. The Watusi (microscopic) cross sections (red line) are plotted against the experimental data (Table 32). 


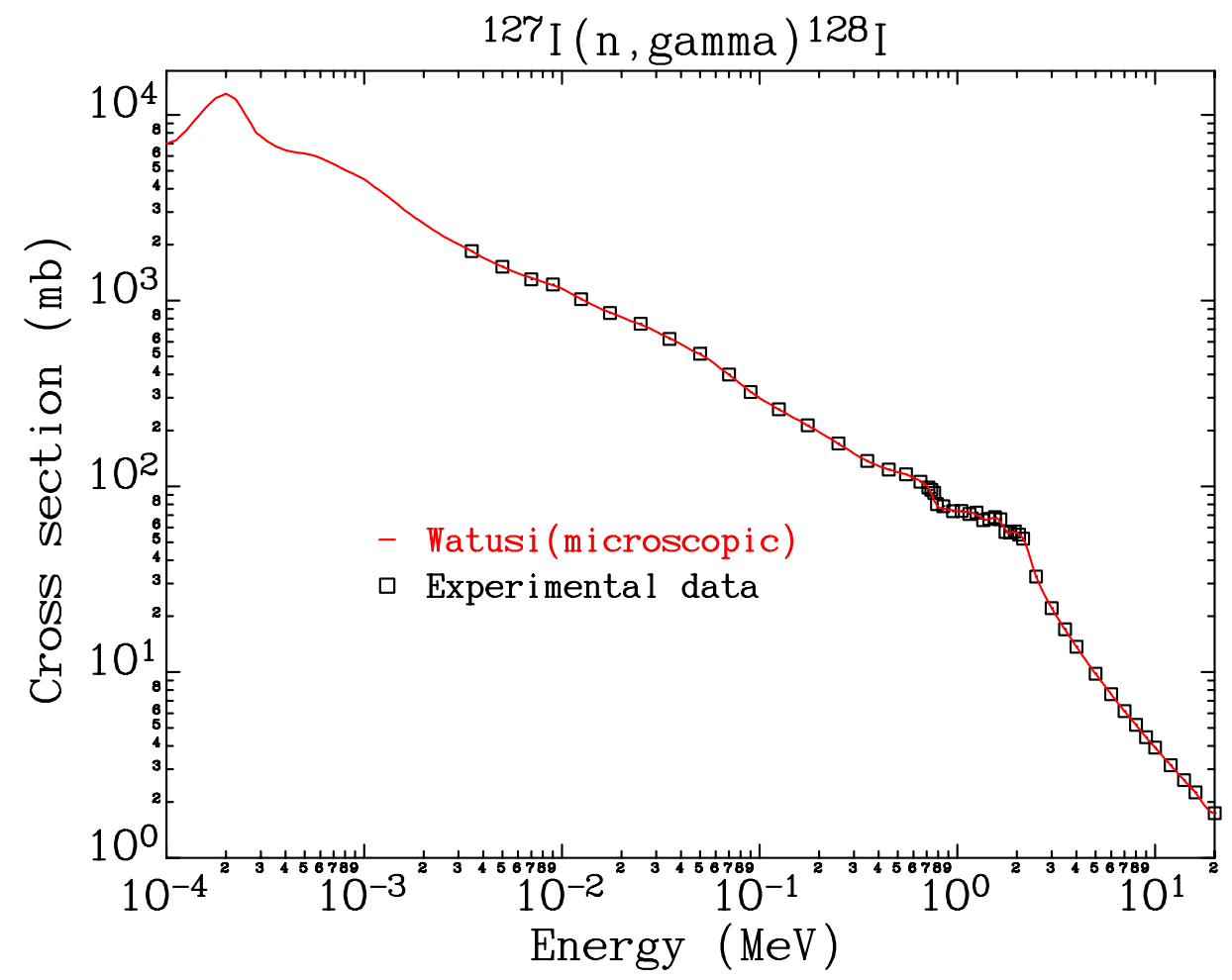

Fig. 27. ${ }^{127} \mathrm{I}(\mathrm{n}, \mathrm{g}){ }^{128} \mathrm{I}$. The Watusi (microscopic) cross sections (red line) are plotted against the experimental data (Tables 33 and 34).

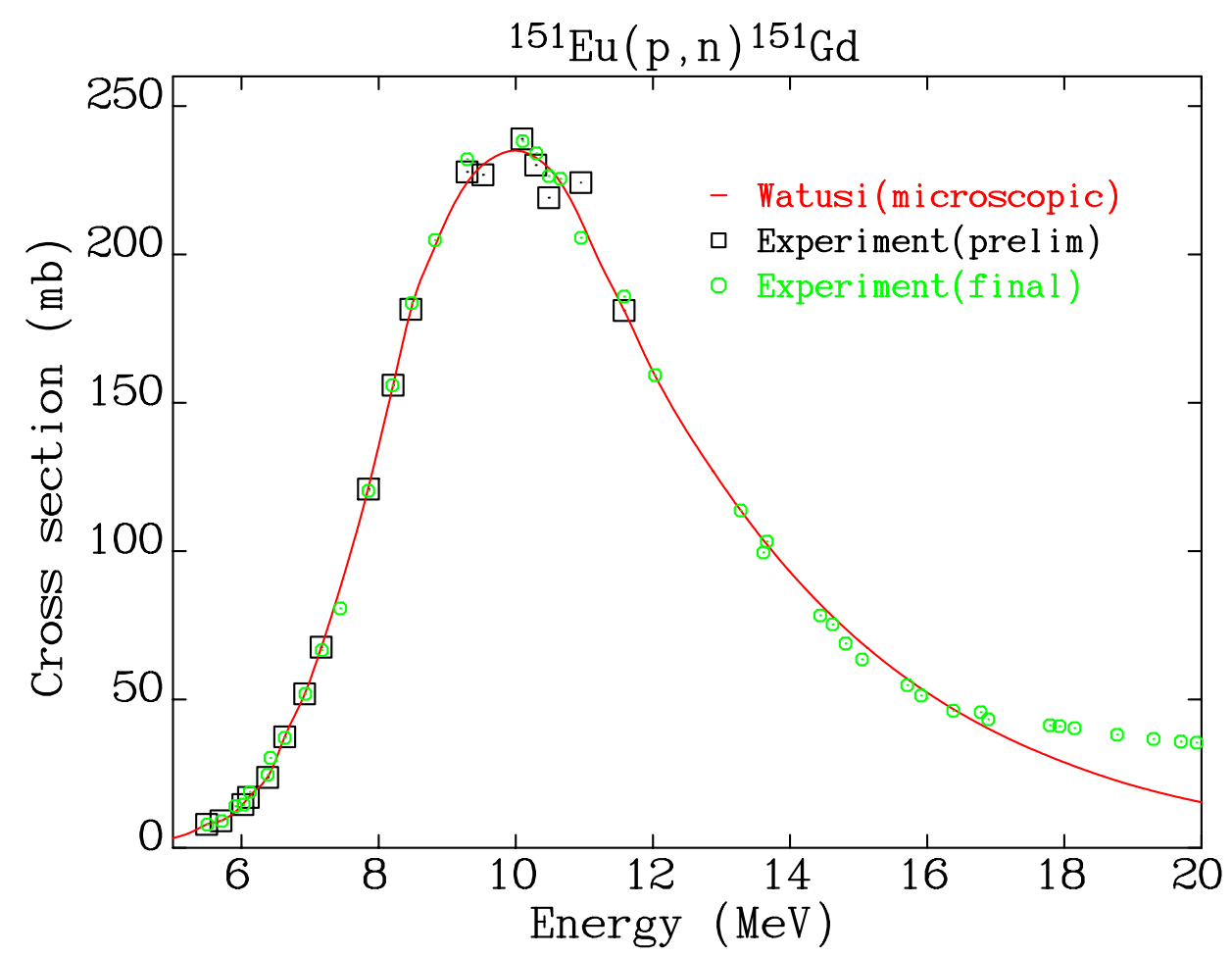

Fig. 28. ${ }^{151} \mathrm{Eu}(\mathrm{p}, \mathrm{n})^{151} \mathrm{Gd}$. The Watusi (microscopic) cross sections (red line) obtained from a fit to the preliminary experimental data (Table 35) are shown against both the preliminary (black squares) and the final (green circles) experimental data (Table 39). 


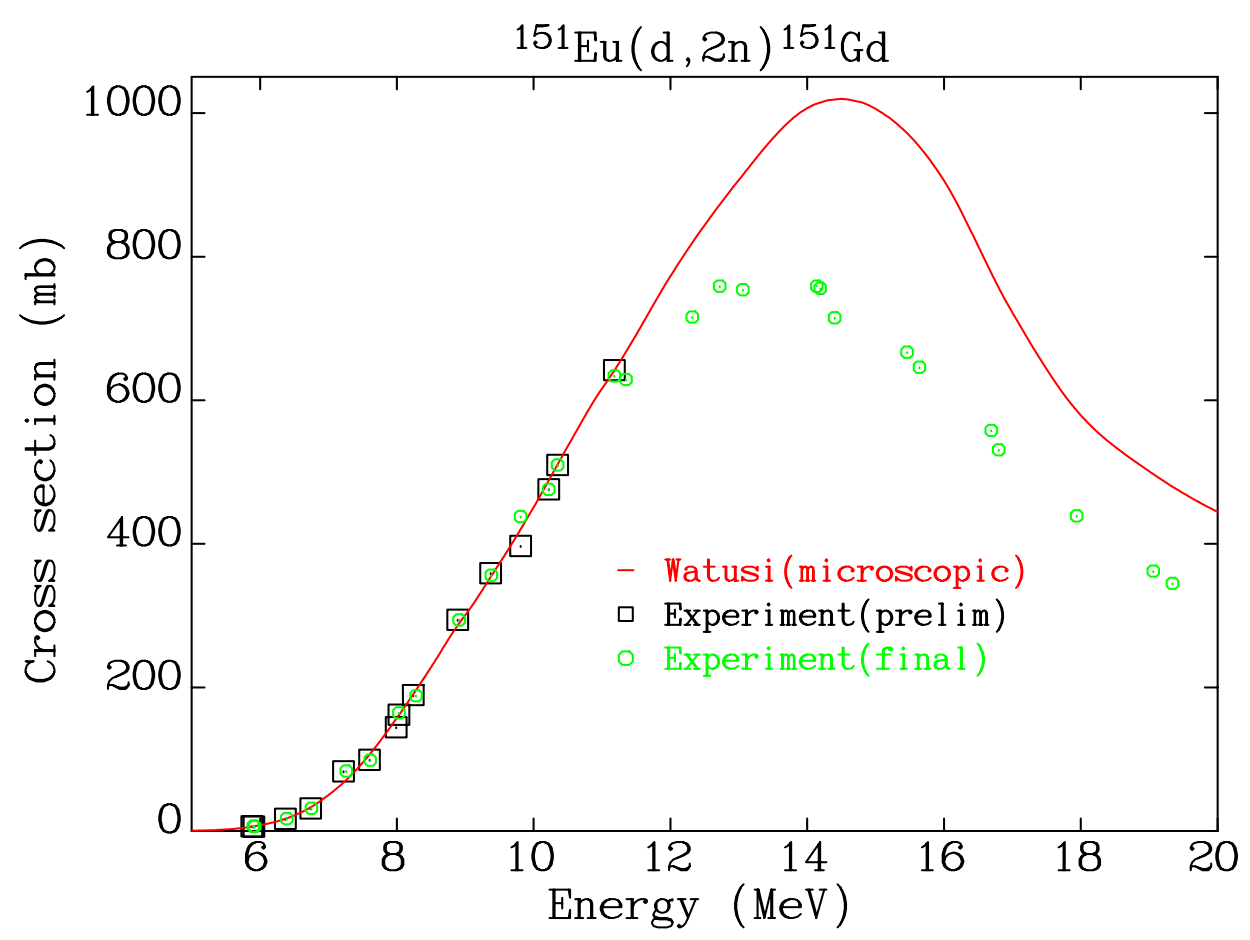

Fig. 29. ${ }^{151} \mathrm{Eu}(\mathrm{d}, 2 \mathrm{n}){ }^{151} \mathrm{Gd}$. The Watusi (microscopic) cross sections (red line) obtained from a fit to the preliminary experimental data (Table 36) are shown against both the preliminary (black squares) and the final (green circles) experimental data (Table 40). Note that the Watusi cross sections above $11 \mathrm{MeV}$ will need revision.

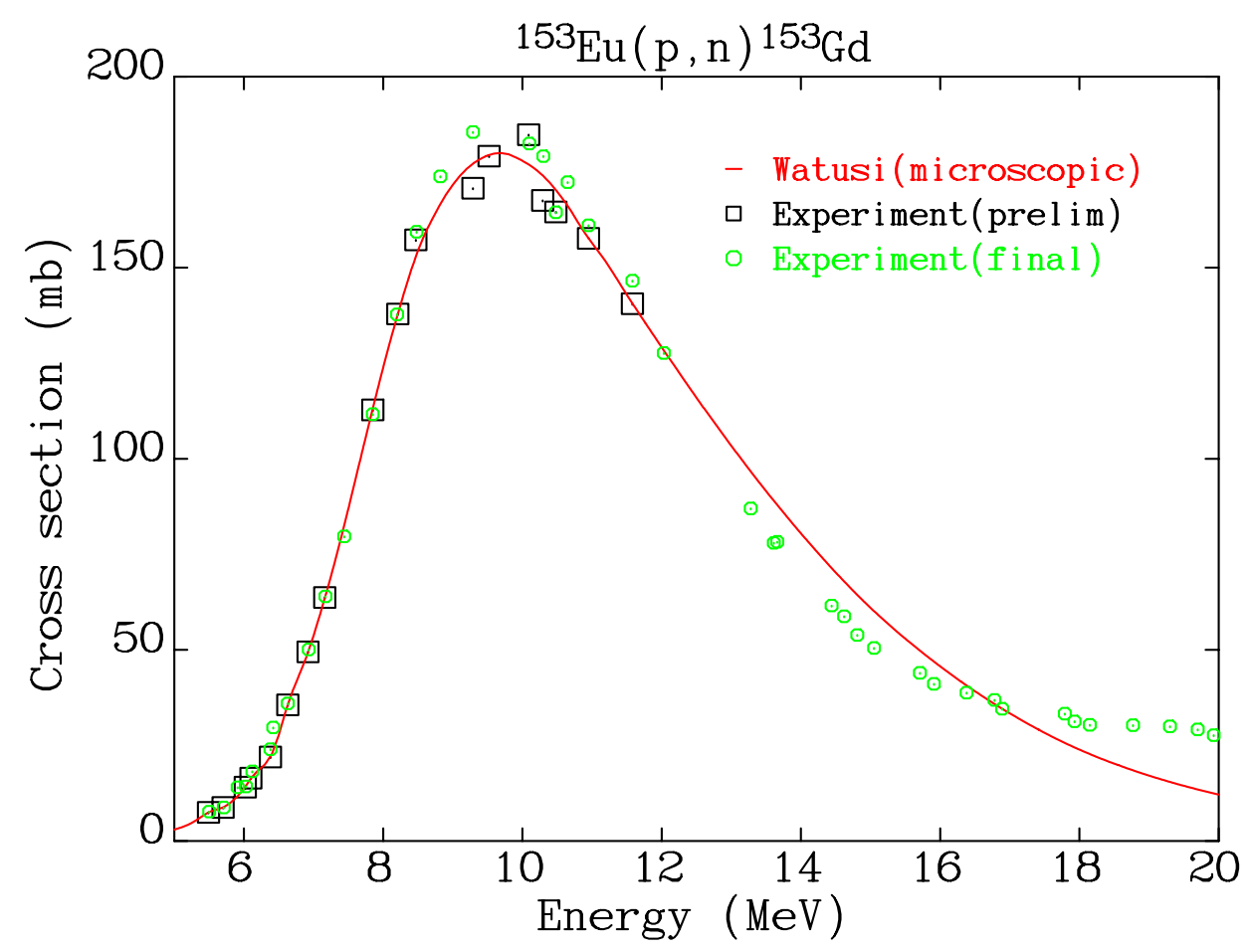

Fig. 30. ${ }^{153} \mathrm{Eu}(\mathrm{p}, \mathrm{n}){ }^{153} \mathrm{Gd}$. The Watusi (microscopic) cross sections (red line) obtained from a fit to the preliminary experimental data (Table 37) are shown against both the preliminary (black squares) and the final (green circles) experimental data (Table 41). Note that the Watusi cross sections above $8 \mathrm{MeV}$ will need revision. 


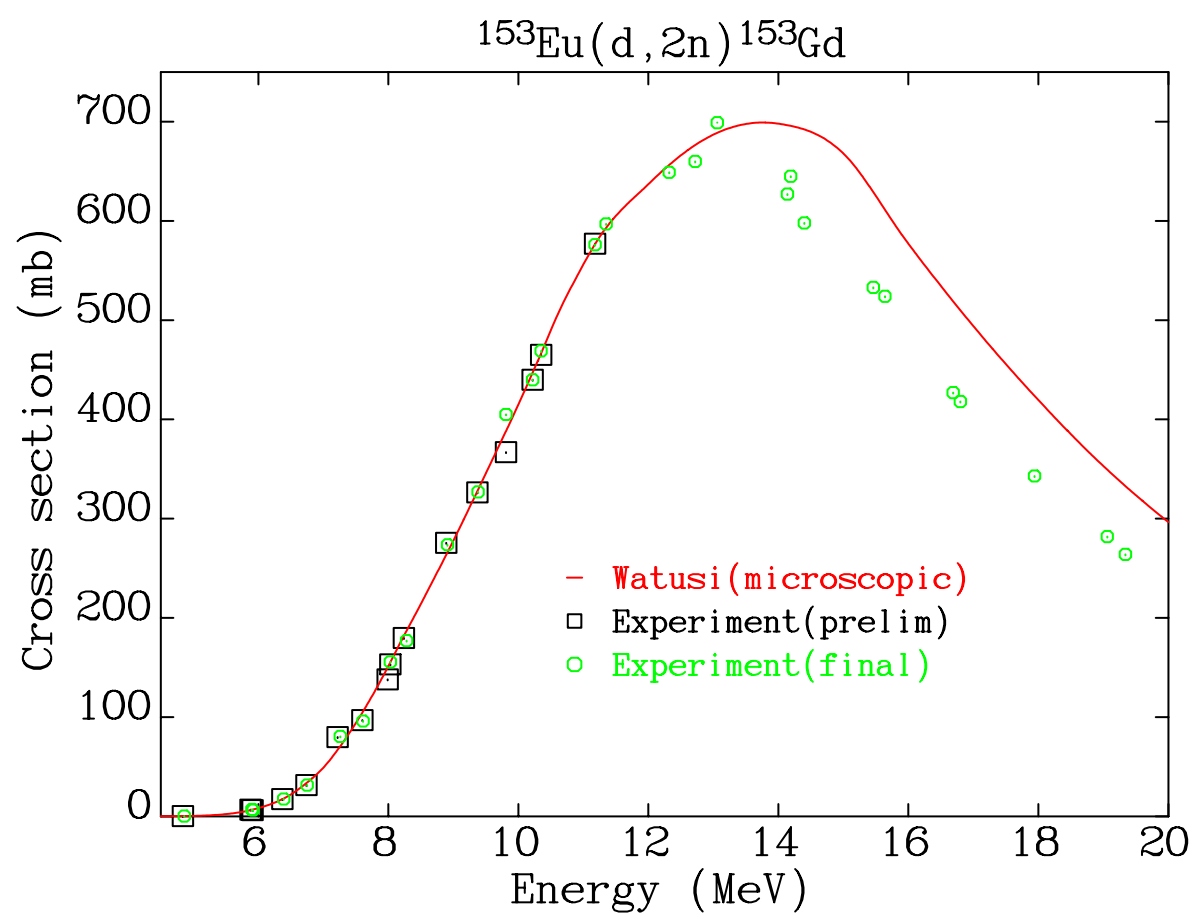

Fig. 31. ${ }^{153} \mathrm{Eu}(\mathrm{d}, 2 \mathrm{n}){ }^{153} \mathrm{Gd}$. The Watusi (microscopic) cross sections (red line) obtained from a fit to the preliminary experimental data (Table 38) are shown against both the preliminary (black squares) and the final (green circles) experimental data (Table 42). Note that the Watusi cross sections above $12 \mathrm{MeV}$ will need revision.

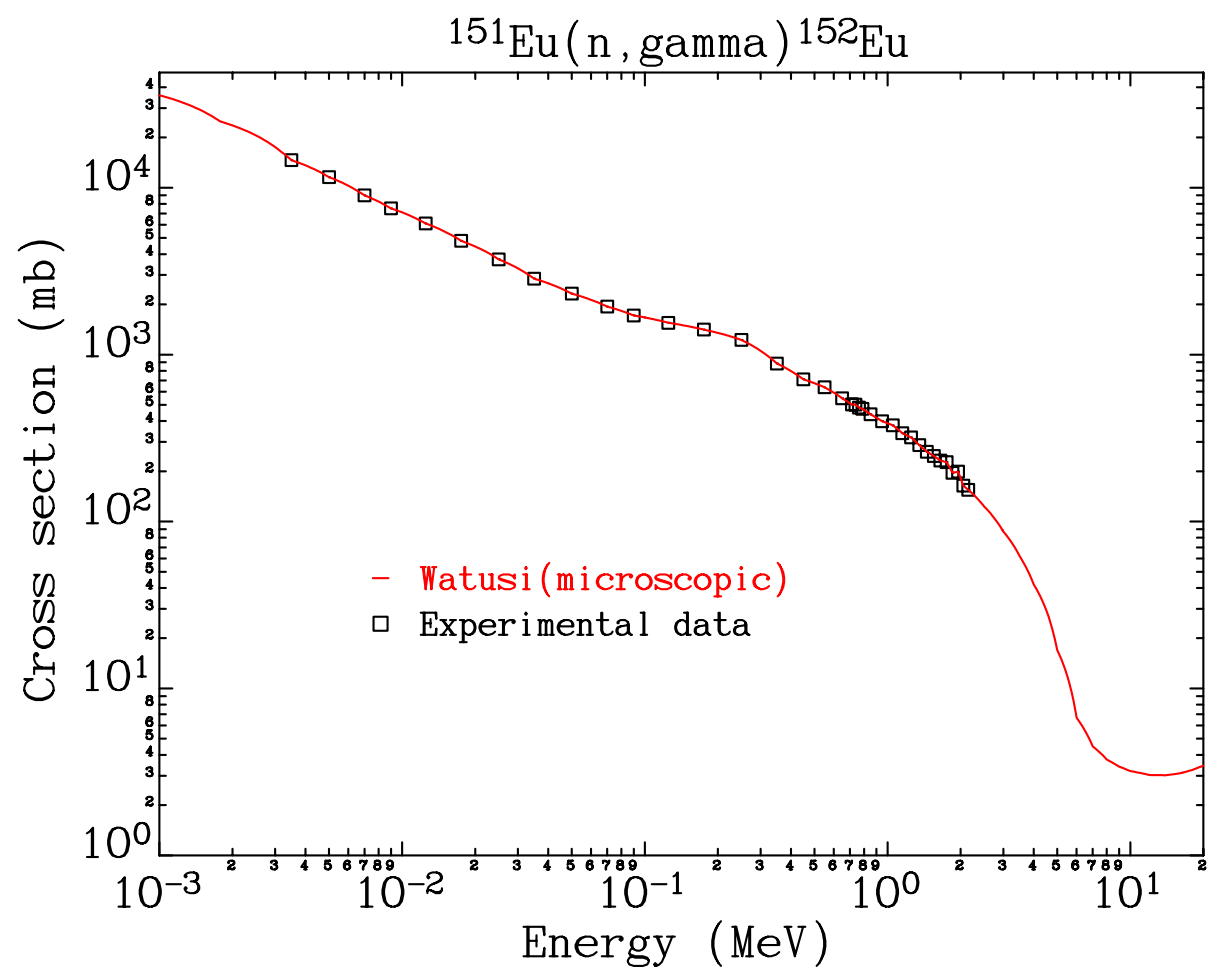

Fig. 32. ${ }^{151} \mathrm{Eu}(\mathrm{n}, \mathrm{g}){ }^{152} \mathrm{Eu}$. The Watusi (microscopic) cross sections (red line) are plotted against the experimental data (Table 43). 


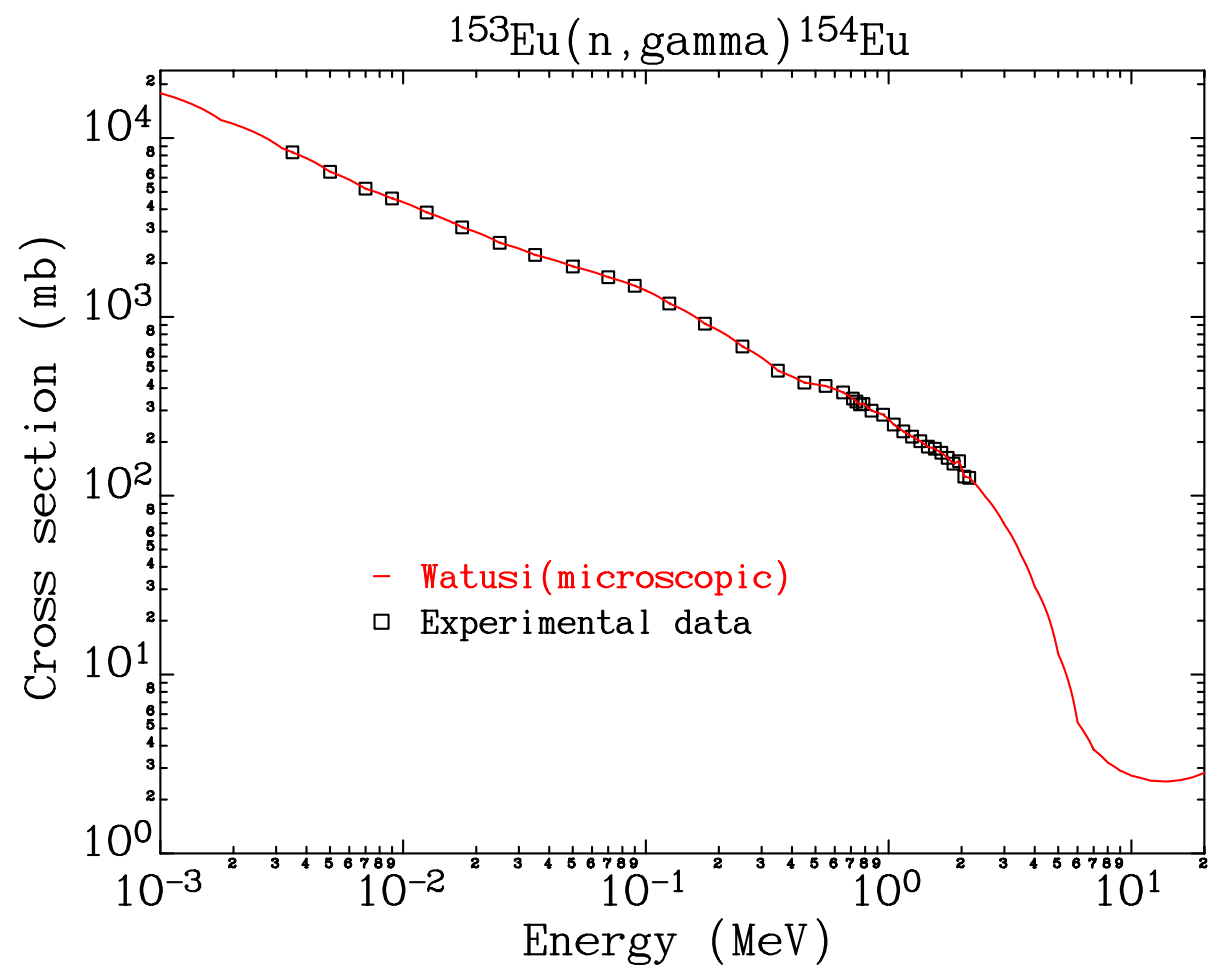

Fig. 33. ${ }^{153} \mathrm{Eu}(\mathrm{n}, \mathrm{g}){ }^{154} \mathrm{Eu}$. The Watusi (microscopic) cross sections (red line) are plotted against the experimental data (Table 44).

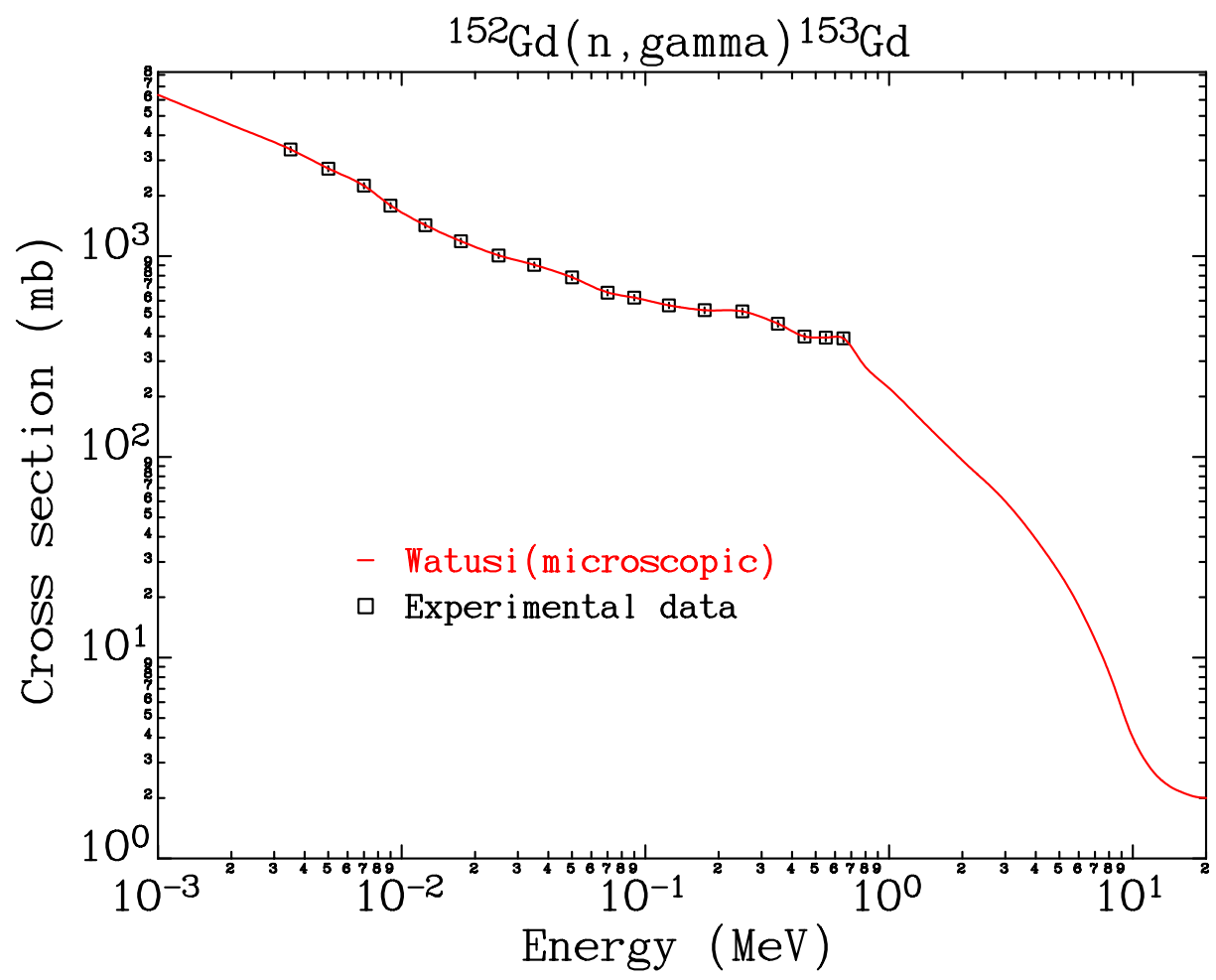

Fig. 34. ${ }^{152} \mathrm{Gd}(\mathrm{n}, \mathrm{g}){ }^{153} \mathrm{Gd}$. The Watusi (microscopic) cross sections (red line) are plotted against the experimental data (Table 45). 


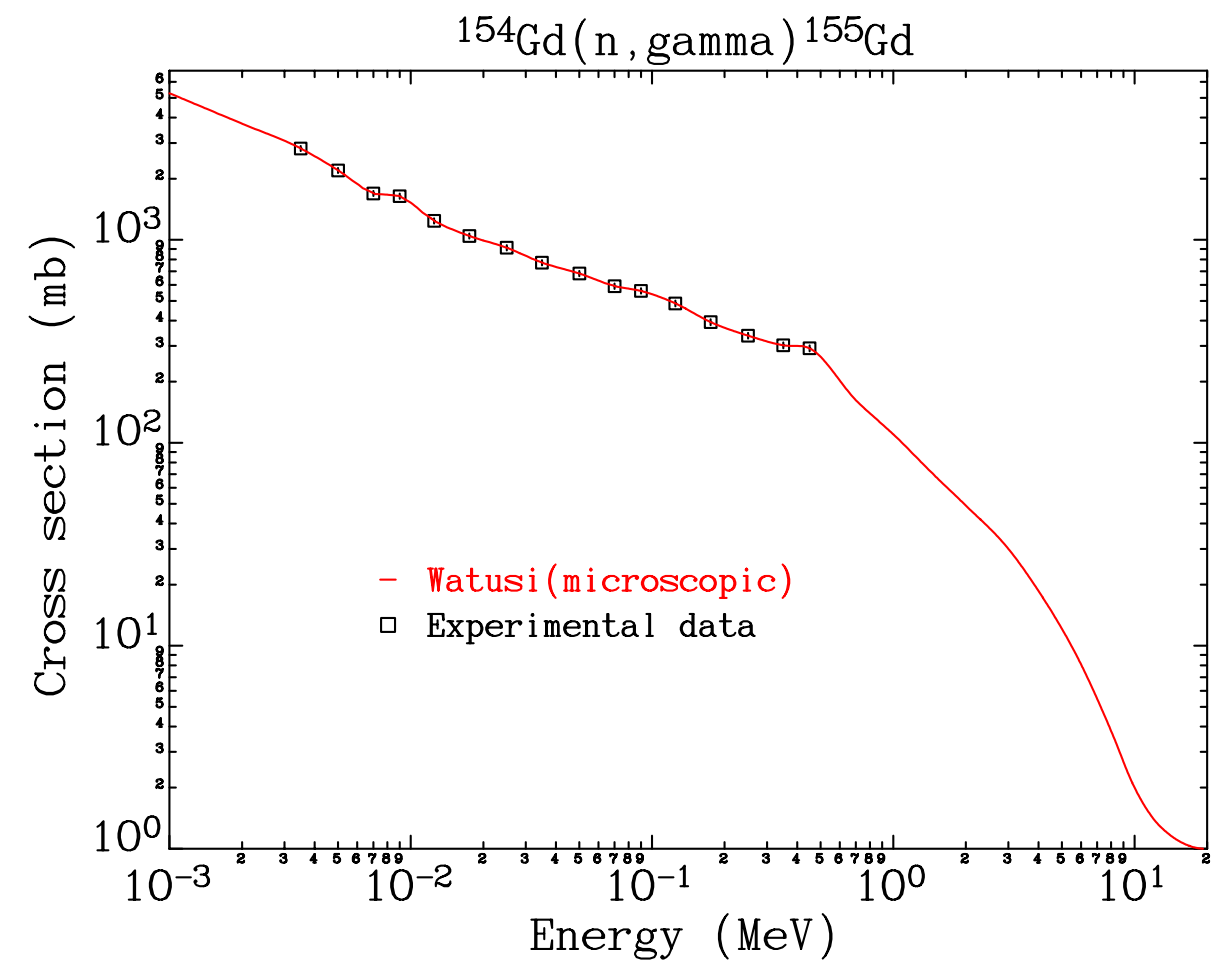

Fig. 35. ${ }^{154} \mathrm{Gd}(\mathrm{n}, \mathrm{g}){ }^{155} \mathrm{Gd}$. The Watusi (microscopic) cross sections (red line) are plotted against the experimental data (Table 46). 